\title{
Anomalies and the Euler characteristic of elliptic Calabi-Yau threefolds
}

\author{
Antonella Grassi and David R. Morrison
}

\begin{abstract}
We investigate the delicate interplay between the types of singular fibers in elliptic fibrations of Calabi-Yau threefolds (used to formulate F-theory) and the "matter" representation of the associated Lie algebra. The main tool is the analysis and the appropriate interpretation of the anomaly formula for six-dimensional supersymmetric theories. We find that this anomaly formula is geometrically captured by a relation among codimension two cycles on the base of the elliptic fibration, and that this relation holds for elliptic fibrations of any dimension. We introduce a "Tate cycle" that efficiently describes this relationship, and which is remarkably easy to calculate explicitly from the Weierstrass equation of the fibration. We check the anomaly cancellation formula in a number of situations and show how this formula constrains the geometry (and in particular the Euler characteristic) of the Calabi-Yau threefold.
\end{abstract}

Traditional compactification of string theory or M-theory on the product of a Calabi-Yau manifold and Minkowski space leads to a low-energy effective theory in which — at least semiclassically — the physics in Minkowski space is encoded by well-known and clearly understood geometric features of the Calabi-Yau manifold.

One of the early lessons in the second superstring revolution was that for the type II theories, as well as M-theory and F-theory, singularities in the Calabi-Yau manifold could lead to interesting and important physical effects. The first of these is non-abelian gauge symmetry of ADE type arising from singularities complex codimension two [1,2]; refinements to the original story show that non-simply-laced as well as simply laced gauge groups can occur [3-7]. The second effect is charged matter in the non-abelian gauge sector $[4,8]$, which arises from the singular structure in complex codimension three. (The codimensions here are codimensions within the Calabi-Yau manifold; in F-theory, one sees the same effects in the base of an elliptic fibration on the Calabi-Yau manifold, where the gauge groups are associated with complex codimension one and the matter with complex codimension two.) 
There are many subtleties involved in determining the physics in this context, and one important guide has been consistency of the induced lowenergy theory. In this paper, we investigate the conditions imposed by anomaly cancellation on F-theory models in six dimensions. There is a delicate interplay between the types of singular fibers in the elliptic fibration used to formulate F-theory, and the corresponding matter representation. We not only check the anomaly cancellation formula (based on an intrinsic geometric analysis of matter from singular fibers, as in $[5,8,9])$ in a number of situations, but we also show how this formula constrains the geometry: given the representation, one can often determine the allowed set of geometries.

We have found a natural setting for our key computation which applies to an elliptically fibered Calabi-Yau manifold of arbitrary dimension. In our setup, for each such manifold we describe both a gauge divisor and a matter cycle (of codimension two) on the base; the anomaly cancellation is the result of a tight relationship between these two.

For an elliptic Calabi-Yau threefold, the associated six-dimensional (6D) quantum field theory is again a gauge theory, and in order for it to be consistent, the gauge, gravitational, and mixed anomalies must vanish. The vanishing of one of these anomalies can be interpreted as a formula for the Euler characteristic of the Calabi-Yau manifold; the vanishing of the others severely constrains the "dictionary" between singular fibers in the elliptic fibration and the matter representation of the gauge theory. Our earlier paper [10] focused on the Euler characteristic formula. In this paper, we will concentrate on the other anomaly cancellation formulas and the correspondence between singular fibers and the matter representation (in our more general setting of a "matter cycle"). We stress that these are closely related.

Interest in these questions has been revived recently by some work on the consistency of low-energy $6 \mathrm{D}$ supergravity theories $[11,12]$. A systematic application of constraints on the low-energy theories, combined with anomaly cancellation, comes fairly close to matching the set of F-theory vacua $[13-15]$.

Moreover, the continued importance of F-theory in constructing string vacua in four dimensions ${ }^{1}$ makes a precise understanding of these issues quite important. The lessons learned from the present study of $6 \mathrm{D}$ models have immediate applications to 4D F-theory models, including those with fluxes and branes. In addition, there are other issues in four dimensions, particularly those related to singularities in complex codimension three in

\footnotetext{
${ }^{1}$ See $[16,17]$ and references therein for recent progress in this direction.
} 
the base of the F-theory elliptic fibration $[18,19]$, whose complete physical understanding will undoubtedly require an understanding of the complex codimension two effects investigated in this paper.

The formula for anomalies is perfectly well-defined in terms of quantities in the quantum field theory, but a complete dictionary between the geometry of the Calabi-Yau manifold and the corresponding quantities in the quantum field theory is not yet known. Thus, our purpose will be to not only verify this formula, but to complete the dictionary with field-theoretic quantities at the same time.

When one of the "type II string theories" is formulated on a ten-manifold of the form $M^{3,1} \times X$ with $X$ a Calabi-Yau threefold and $M^{3,1}$ a flat spacetime of dimension four, the resulting theory has a low-energy approximation, which takes the form of a $4 \mathrm{D}$ quantum field theory with quite realistic physical properties (depending on certain properties of the Calabi-Yau threefold).

Elliptic Calabi-Yau threefolds with a section $\pi: X \rightarrow B$ have also been used in a different way in string theory. We can ask what happens to the type IIA theory in the limit in which the Calabi-Yau metric on $X$ is varied, so that the fibers of the map $\pi$ shrink to zero area and the string coupling approaches infinity. It turns out that the resulting physical theory has a low energy approximation which takes the form of a $6 \mathrm{D}$ quantum field theory. This limiting theory can also be described more directly in terms of the periods $\tau(b)$ of the elliptic curves $\pi^{-1}(b)$, regarded as a multi-valued function on $B$. The type IIB string theory is compactified on $B$ with the aid of this function, using what are known as D-branes along the discriminant locus of the map $\pi$. (This latter approach is known as "F-theory.")

This 6D quantum field theory includes gravity as well as a gauge field theory whose gauge group is the compact reductive group $G$ defined in Section 2.

The gauge theory is on a $8 \mathrm{D}$ manifold with boundary, $Y$, whose boundary is $M^{3,1} \times S^{1} ; Y$ is equipped with a principal $G$-bundle $\mathcal{G}$ (the "gauge bundle"). Then the curvature $F$ of the gauge connection is an $\operatorname{ad}(\mathcal{G})$-valued two-form, where each fiber $\operatorname{ad}(\mathcal{G})_{x}$ of $\operatorname{ad}(\mathcal{G})$ is isomorphic to the Lie algebra $\mathfrak{g}$ of $G$, with $\mathcal{G}_{x}$ acting on $\operatorname{ad}(\mathcal{G})_{x}$ via the adjoint action of $G$ on $\mathfrak{g}$. Similarly, $Y$ is equipped with a (pseudo-)Riemannian metric, and the curvature $R$ of the Levi-Civita connection is a two-form taking values in the endomorphisms of the tangent bundle. (Notations and background can be found in Appendix A.)

In order to be a consistent quantum theory, the "anomalies" of this theory must vanish. In particular, Schwarz shows [20] that in these model, $(\mathcal{N}=1$ supersymmetric theories in six dimensions with a reductive gauge 
group $G$ ), the anomalies are characterized by an eight-form, made from curvatures and gauge field two-forms.

We now discuss the content of each section of the paper, although not in the order they actually appear:

Sections 5 to 7 are the pivot around which the paper unfolds: we describe in Section 5 the anomaly cancellations coming from physics and recast them in the geometric language of codimension two cycles and representations in the F-theory set up. This is summarized in Equations (5.3) to (5.6) and expressed in geometric terms in Sections 6 and 7. One upshot is that through the anomalies the representation theory puts constraints on the possible F-theory geometries and vice versa.

In the following Sections 8 and 9, we describe in concrete terms how this works for the standard "generic" codimension two singularities of elliptic fibrations from [4] (as already verified in [10]) as well as for some new codimension two singularities such as the one from [21]. We also introduce some singularities that are considered here for the first time. In the sections preceding Section 5, we introduce the language and the concepts that are necessary to properly state and understand the geometric interpretations of the anomaly.

Some key features of the anomaly cancellation are phrased in terms of the Casimir operators of a (real) reductive Lie algebra $\mathfrak{g}$. These are discussed in Section 1. Motivated by the anomalies we say that two representations $\rho$ and $\rho^{\prime}$ are Casimir equivalent in degrees 2 and 4 if $\operatorname{Tr}_{\rho} F^{k}=\operatorname{Tr}_{\rho^{\prime}} F^{k}$ for $k=2,4$. In fact, since the anomalies we consider only involve these degree 2 and 4 quantities, we can freely replace a representation with a Casimirequivalent one without affecting the validity of anomaly cancellation, as described in table 3. Several examples of this equivalence will be important for us in this paper. In the following Section 2, we explain how a gauge algebra can be naturally associated with an elliptic fibration with section, via its Weierstrass equation, Kodaira's classification of singular fibers and Tate's algorithm; this is summarized in table 4.

An elliptic fibration with a base of dimension at least two also has a "matter representation" (i.e., the matter representation appropriate to F-theory or M-theory), with contributions from the components of the discriminant locus of the elliptic fibration and from the singular locus of the discriminant locus $\Sigma$. The matter representation for $6 \mathrm{D}$ theories gets contributions from rational curves which are components of fibers in the Calabi-Yau resolution of a Weierstrass elliptic fibration. We describe how to compute this in Section 3 and introduce the virtual matter cycle, which is an element of the Chow group with coefficients in the representation ring 
of the gauge algebra $\mathfrak{g}$; the representations are derived from the branching rules. (In Section 7, we show that anomaly cancellation for these $6 \mathrm{D}$ theories follows from a relation among algebraic cycles. If the virtual matter cycle is rationally equivalent to another cycle, the Tate cycle, and some conditions on the intersections of the components of the discriminant locus hold, then the anomalies vanish; this is actually verified in last two sections of the paper.)

The Tate cycle is introduced in Equation (4.4) in Section 4. The constituents of this cycle are found in tables 8 and 9: the first are derived from the Tate algorithm, whereas the latter are mostly derived from the branching rules, except some which are substituted by their Casimir equivalent: this substitution is crucial for the verification of anomaly cancellation. In fact, in Section 9 for each type of singular point, we compute the contribution to the Tate cycle and compare it to the contribution to the matter cycle.

We wish to emphasize that the Tate cycle is very easy to compute from the Weierstrass equation, and gives a very quick route to determining the matter representation up to Casimir equivalence. Existing techniques for determining the matter representation more precisely are much more complicated, and involve either making a group-theory analysis of each singular point (as in [8]), or constructing a resolution of singularities explicitly (as in $[22,23])$.

\section{Casimir operators}

Some key features of the anomaly cancellation are phrased in terms of the Casimir operators of a (real) reductive Lie algebra $\mathfrak{g}$ of compact type. Recall that these are complex-valued polynomial functions on $\mathfrak{g}$, which are invariant under the adjoint action of $\mathfrak{g}$ on itself. By the basic structure theory for reductive Lie algebras (cf. $[24,25]$ ), if we choose a Cartan subalgebra $\mathfrak{h} \subset \mathfrak{g}_{\mathbb{C}}$ of the complexification $\mathfrak{g}_{\mathbb{C}}$ of $\mathfrak{g}$, then the Casimir operators can be identified with elements of the ring $S\left(\mathfrak{h}^{*}\right)^{W}$, where $S\left(\mathfrak{h}^{*}\right)$ is the ring of polynomial functions on $\mathfrak{h}$, and $W$ is the Weyl group of $\mathfrak{g}$.

Useful examples of Casimir operators are given by $F \mapsto \operatorname{Tr}_{\rho} F^{k}$ where $\rho$ is a finite-dimensional complex representation of $\mathfrak{g}, k$ is a positive integer, and $F \in \mathfrak{g}$. The anomalies of the $6 \mathrm{D}$ theories we study only involve Casimir operators of degrees 2 and 4; examples of the latter are provided by $F \mapsto$ $\left(\operatorname{Tr}_{\rho} F^{2}\right)^{2}$ and $F \mapsto \operatorname{Tr}_{\rho} F^{4}$. We often shorten the notation and simply refer to these operators as $\left(\operatorname{Tr}_{\rho} F^{2}\right)^{2}$ and $\operatorname{Tr}_{\rho} F^{4}$, respectively.

For any simple non-abelian Lie algebra $\mathfrak{g}$, the space of Casimir operators of degree 2 is $1 \mathrm{D}$, spanned by $\operatorname{Tr}_{\rho} F^{2}$ for any non-trivial representation $\rho$. 
Table 1: Normalized traces for the exceptional algebras.

\begin{tabular}{lccccc}
\hline $\mathfrak{g}$ & $\mathfrak{e}_{6}$ & $\mathfrak{e}_{7}$ & $\mathfrak{e}_{8}$ & $\mathfrak{f}_{4}$ & $\mathfrak{g}_{2}$ \\
\hline $\operatorname{tr} F^{2}$ & $\frac{1}{6} \operatorname{Tr}_{\mathbf{2 7}} F^{2}$ & $\frac{1}{12} \operatorname{Tr}_{\mathbf{5 6}} F^{2}$ & $\frac{1}{60} \operatorname{Tr}_{\text {adj }} F^{2}$ & $\frac{1}{6} \operatorname{Tr}_{\mathbf{2 6}} F^{2}$ & $\frac{1}{2} \operatorname{Tr}_{\mathbf{7}} F^{2}$ \\
\hline
\end{tabular}

Our main result will be formulated with the aid of a specific basis for this 1D space, given by a particular Casimir operator of degree 2, which we will denote by $F \mapsto \operatorname{tr} F^{2}$, or simply $\operatorname{tr} F^{2}$ (using lowercase tr to distinguish this particular "normalized" trace). The normalization we use is a natural one, introduced in [26] (see also [14]): if $G$ is the compact simple simply connected Lie group whose Lie algebra is $\mathfrak{g}$, and if we choose an appropriate generator of $\pi_{3}(G) \cong \mathbb{Z}$, then for any group homomorphism $\varphi: \operatorname{SU}(2) \rightarrow G$ whose homotopy class is $N$ times the generator, the integral

$$
\frac{1}{32 \pi^{2}} \int \operatorname{tr}(d \varphi(F) \wedge d \varphi(F))
$$

evaluates to $N$ when $F$ is the curvature of (any) 4D SU(2)-instanton with instanton number 1 , and $d \varphi: \mathfrak{s u}(2) \rightarrow \mathfrak{g}$ is the induced Lie algebra homomorphism. The choice of generator of $\pi_{3}(G)$ used in this definition (i.e., the sign) is determined by the additional requirement that $\operatorname{Tr}_{\rho} F^{2}$ should be a positive multiple of $\operatorname{tr} F^{2}$ for any non-trivial irreducible representation $\rho$.

This "normalized trace" has been determined for all of the simple nonabelian algebras, in terms of traces in familiar representations. For the exceptional simple algebras, the normalized trace is described in table 1 in terms of the trace in the irreducible representation of smallest dimension. (We label irreducible representations of the exceptional simple algebras by their dimension, in bold face.) For $\mathfrak{s u}(m)$ (resp. $\mathfrak{s p}(n)$ ), we have $\operatorname{tr} F^{2}=\operatorname{Tr}_{\text {fund }} F^{2}$ in terms of the fundamental representation "fund", i.e., the standard representation of complex dimension $m$ (resp. $2 n)$. For $\mathfrak{s o}(\ell)$, we have $\operatorname{tr} F^{2}=\frac{1}{2} \operatorname{Tr}_{\text {vect }} F^{2}$ in terms of the vector representation "vect" (i.e., the standard real representation of real dimension $\ell$ ). In addition, for any positive integer $k$ we define $\operatorname{tr} F^{k}=\operatorname{Tr}_{\text {fund }} F^{k}$ when $\mathfrak{g} \cong \mathfrak{s u}(m)$ or $\mathfrak{s p}(n)$, and $\operatorname{tr} F^{k}=\frac{1}{2} \operatorname{Tr}_{\text {vect }} F^{k}$ when $\mathfrak{g} \cong \mathfrak{s o}(\ell)$; this agrees with the facts about tr stated above in the case $k=2$.

For $\mathfrak{g}=\mathfrak{s u}(2), \mathfrak{s u}(3), \mathfrak{g}_{2}, \mathfrak{f}_{4}, \mathfrak{e}_{6}, \mathfrak{e}_{7}$ or $\mathfrak{e}_{8}$, the space of Casimir operators of degree 4 is $1 \mathrm{D}$, and $\left(\operatorname{tr} F^{2}\right)^{2}$ provides a natural basis. For all other simple algebras except $\mathfrak{s o}(8)$, the space of Casimir operators of degree 4 is $2 \mathrm{D}$, with $\left(\operatorname{tr} F^{2}\right)^{2}$ and $\operatorname{tr} F^{4}$ providing a basis. For $\mathfrak{s o}(8)$, the space of Casimir operators of degree 4 is $3 \mathrm{D}$, and we can take $\operatorname{Tr}_{\text {vect }} F^{4}, \operatorname{Tr}_{\text {spin }_{+}} F^{4}$ and $\operatorname{Tr}_{\text {spin }} F^{4}$ 
as a basis for this space, where vect, spin $_{+}$and spin $\_$are the three $8 \mathrm{D}$ representations of $\mathfrak{s o}(8)$ (permuted by triality). The key relation in this latter case is

$$
\left(\operatorname{tr} F^{2}\right)^{2}=\frac{1}{3} \operatorname{Tr}_{\text {vect }} F^{4}+\frac{1}{3} \operatorname{Tr}_{\text {spin }_{+}} F^{4}+\frac{1}{3} \operatorname{Tr}_{\text {spin }_{-}} F^{4}
$$

(verified in Appendix C).

For any representation $\rho$ of a simple algebra $\mathfrak{g}$, we can then express $\operatorname{Tr}_{\rho} F^{2}$ as a multiple of $\operatorname{tr} F^{2}$ and $\operatorname{Tr}_{\rho} F^{4}$ as a linear combination of the basis elements chosen above. For the representations we consider here, much of this data was worked out by Erler [27], based in part on the previous work of van Nieuwenhuizen [28]. We collect the information we need ${ }^{2}$ in table 2. In addition to the adjoint representation, and the fundamental and vector representations mentioned above, our analysis includes the anti-symmetric powers $\Lambda^{k}$ of the fundamental representation in the case of $\mathfrak{s u}(m)$, the nontrivial irreducible component $\Lambda_{\text {irr }}^{k}$ of the $k$ th anti-symmetric power of the fundamental representation in the case of $\mathfrak{s p}(n)$, and the spinor representations $\operatorname{spin}_{*}$ in the case of $\mathfrak{s o}\left(\ell\right.$ ). (We use the notation $\operatorname{spin}_{*}$ to denote either the unique spinor representation "spin" when $\ell$ is odd, or either of the two spinor representations " $\operatorname{spin}_{ \pm}$" when $\ell$ is even.)

We say that two representations $\rho$ and $\rho^{\prime}$ are Casimir equivalent in degrees 2 and 4 if $\operatorname{Tr}_{\rho} F^{k}=\operatorname{Tr}_{\rho^{\prime}} F^{k}$ for $k=2$, 4. Since the the anomalies we consider only involve these degree 2 and 4 quantities, we can freely replace a representation with a Casimir-equivalent one without affecting the validity of anomaly cancellation. Two examples of this equivalence will be important for us in this paper. First, for $\mathfrak{s u}(m), m \geq 6$, the representation $\rho_{m}=\Lambda^{3} \oplus$ (fund $)^{\oplus\left(m^{2}-7 m+10\right) / 2}$ has invariants

$$
\begin{aligned}
\operatorname{Tr}_{\rho_{m}} F^{2}= & \left(\frac{1}{2}\left(m^{2}-5 m+6\right)+\frac{1}{2}\left(m^{2}-7 m+10\right)\right) \operatorname{tr} F^{2} \\
= & (m-4)(m-2) \operatorname{tr} F^{2} \\
\operatorname{Tr}_{\rho_{m}} F^{4}= & ((3 m-12)+0)\left(\operatorname{tr} F^{2}\right)^{2}+\left(\frac{1}{2}\left(m^{2}-17 m+54\right)\right. \\
& \left.+\frac{1}{2}\left(m^{2}-7 m+10\right)\right) \operatorname{tr} F^{4} \\
= & 3(m-4)\left(\operatorname{tr} F^{2}\right)^{2}+(m-4)(m-8) \operatorname{tr} F^{4},
\end{aligned}
$$

\footnotetext{
${ }^{2}$ We have included a complete derivation of the data for $\mathfrak{s o}(\ell)$ in Appendix C, including the case $\ell=8$, since the special features of that case seem to have been overlooked in the literature.
} 
Table 2: Casimir operators of degrees 2 and 4 .

\begin{tabular}{|c|c|c|c|}
\hline$\underline{\mathfrak{g}}$ & $\rho$ & $\operatorname{Tr}_{\rho} F^{2}$ & $\operatorname{Tr}_{\rho} F^{4}$ \\
\hline $\mathfrak{s u}(m)$ & $\operatorname{adj}$ & $2 m \operatorname{tr} F^{2}$ & $(m+6)\left(\operatorname{tr} F^{2}\right)^{2}$ \\
\hline$m=2,3$ & fund & $\operatorname{tr} F^{2}$ & $\frac{1}{2}\left(\operatorname{tr} F^{2}\right)^{2}$ \\
\hline $\mathfrak{s u}(m)$ & $\operatorname{adj}$ & $2 m \operatorname{tr} F^{2}$ & $6\left(\operatorname{tr} F^{2}\right)^{2}+2 m \operatorname{tr} F^{4}$ \\
\hline \multirow[t]{4}{*}{$m \geq 4$} & fund & $\operatorname{tr} F^{2}$ & $0\left(\operatorname{tr} F^{2}\right)^{2}+\operatorname{tr} F^{4}$ \\
\hline & $\Lambda^{2}$ & $(m-2) \operatorname{tr} F^{2}$ & $3\left(\operatorname{tr} F^{2}\right)^{2}+(m-8) \operatorname{tr} F^{4}$ \\
\hline & $\Lambda^{3}$ & $\frac{1}{2}\left(m^{2}-5 m+6\right) \operatorname{tr} F^{2}$ & $(3 m-12)\left(\operatorname{tr} F^{2}\right)^{2}$ \\
\hline & & & $+\frac{1}{2}\left(m^{2}-17 m+54\right) \operatorname{tr} F^{4}$ \\
\hline $\mathfrak{s u}(8)$ & $\Lambda^{4}$ & $20 \operatorname{tr} F^{2}$ & $18\left(\operatorname{tr} F^{2}\right)^{2}-16 \operatorname{tr} F^{4}$ \\
\hline $\mathfrak{s p}(n)$, & $\operatorname{adj}$ & $(2 n+2) \operatorname{tr} F^{2}$ & $3\left(\operatorname{tr} F^{2}\right)^{2}+(2 n+8) \operatorname{tr} F^{4}$ \\
\hline \multirow[t]{3}{*}{$n \geq 2$} & fund & $\operatorname{tr} F^{2}$ & $0\left(\operatorname{tr} F^{2}\right)^{2}+\operatorname{tr} F^{4}$ \\
\hline & $\Lambda_{\mathrm{irr}}^{2}$ & $(2 n-2) \operatorname{tr} F^{2}$ & $3\left(\operatorname{tr} F^{2}\right)^{2}+(2 n-8) \operatorname{tr} F^{4}$ \\
\hline & $\Lambda_{\mathrm{irr}}^{3}$ & $\left(2 n^{2}-5 n+2\right) \operatorname{tr} F^{2}$ & $\begin{array}{c}(6 n-12)\left(\operatorname{tr} F^{2}\right)^{2}+\left(2 n^{2}-17 n\right. \\
+26) \operatorname{tr} F^{4}\end{array}$ \\
\hline $\mathfrak{s p}(4)$ & $\Lambda_{\mathrm{irr}}^{4}$ & $14 \operatorname{tr} F^{2}$ & $15\left(\operatorname{tr} F^{2}\right)^{2}-16 \operatorname{tr} F^{4}$ \\
\hline $\mathfrak{s o}(\ell)$ & $\operatorname{adj}$ & $(2 \ell-4) \operatorname{tr} F^{2}$ & $12\left(\operatorname{tr} F^{2}\right)^{2}+(2 \ell-16) \operatorname{tr} F^{4}$ \\
\hline$\ell \geq 7$ & vect & $2 \operatorname{tr} F^{2}$ & $0\left(\operatorname{tr} F^{2}\right)^{2}+2 \operatorname{tr} F^{4}$ \\
\hline$\ell \neq 8$ & $\operatorname{spin}_{*}$ & $\operatorname{dim}\left(\operatorname{spin}_{*}\right)\left(\frac{1}{4} \operatorname{tr} F^{2}\right)$ & $\operatorname{dim}\left(\operatorname{spin}_{*}\right)\left(\frac{3}{16}\left(\operatorname{tr} F^{2}\right)^{2}-\frac{1}{8} \operatorname{tr} F^{4}\right)$ \\
\hline \multirow[t]{4}{*}{$\mathfrak{s o}(8)$} & $\operatorname{adj}$ & $12 \operatorname{tr} F^{2}$ & $\begin{array}{c}4 \operatorname{Tr}_{\mathrm{vect}} F^{4}+4 \operatorname{Tr}_{\mathrm{spin}_{+}} F^{4} \\
+4 \operatorname{Tr}_{\text {spin }_{-}} F^{4}\end{array}$ \\
\hline & vect & $2 \operatorname{tr} F^{2}$ & $\operatorname{Tr}_{\mathrm{vect}} F^{4}$ \\
\hline & $\operatorname{spin}_{+}$ & $2 \operatorname{tr} F^{2}$ & $\operatorname{Tr}_{\text {spin }_{+}} F^{4}$ \\
\hline & $\operatorname{spin}_{-}$ & $2 \operatorname{tr} F^{2}$ & $\operatorname{Tr}_{\text {spin }} F^{4}$ \\
\hline \multirow[t]{2}{*}{$\mathfrak{e}_{6}$} & $\operatorname{adj}$ & $24 \operatorname{tr} F^{2}$ & $18\left(\operatorname{tr} F^{2}\right)^{2}$ \\
\hline & 27 & $6 \operatorname{tr} F^{2}$ & $3\left(\operatorname{tr} F^{2}\right)^{2}$ \\
\hline \multirow[t]{2}{*}{$\mathfrak{e}_{7}$} & $\operatorname{adj}$ & $36 \operatorname{tr} F^{2}$ & $24\left(\operatorname{tr} F^{2}\right)^{2}$ \\
\hline & 56 & $12 \operatorname{tr} F^{2}$ & $6\left(\operatorname{tr} F^{2}\right)^{2}$ \\
\hline $\mathfrak{e}_{8}$ & $\operatorname{adj}$ & $60 \operatorname{tr} F^{2}$ & $36\left(\operatorname{tr} F^{2}\right)^{2}$ \\
\hline \multirow[t]{2}{*}{$\mathfrak{f}_{4}$} & $\operatorname{adj}$ & $18 \operatorname{tr} F^{2}$ & $15\left(\operatorname{tr} F^{2}\right)^{2}$ \\
\hline & 26 & $6 \operatorname{tr} F^{2}$ & $3\left(\operatorname{tr} F^{2}\right)^{2}$ \\
\hline \multirow[t]{2}{*}{$\mathfrak{g}_{2}$} & $\operatorname{adj}$ & $8 \operatorname{tr} F^{2}$ & $10\left(\operatorname{tr} F^{2}\right)^{2}$ \\
\hline & 7 & $2 \operatorname{tr} F^{2}$ & \\
\hline
\end{tabular}


Table 3: Casimir equivalences.

\begin{tabular}{lc}
\hline $\mathfrak{g}$ & $\Lambda^{3} \oplus$ fund $^{\oplus 2} \sim\left(\Lambda^{2}\right)^{\oplus 2}$ \\
$\mathfrak{s u}(6)$ & $\Lambda^{3} \oplus$ fund $^{\oplus 5} \sim\left(\Lambda^{2}\right)^{\oplus 3}$ \\
$\mathfrak{s u}(7)$ & $\Lambda^{3} \oplus$ fund $^{\oplus 9} \sim\left(\Lambda^{2}\right)^{\oplus 4}$ \\
$\mathfrak{s u}(8)$ & $\Lambda^{4} \oplus$ fund $^{\oplus 16} \sim\left(\Lambda^{2}\right)^{\oplus 6}$ \\
$\mathfrak{s u}(8)$ & $\Lambda_{\text {irr }}^{3} \oplus$ fund $^{\oplus 3} \sim\left(\Lambda_{\text {irr }}^{2}\right)^{\oplus 2}$ \\
$\mathfrak{s p}(3)$ & $\Lambda_{\text {irr }}^{3} \oplus$ fund $^{\oplus 10} \sim\left(\Lambda_{\text {irr }}^{2}\right)^{\oplus 4}$ \\
$\mathfrak{s p}(4)$ & $\Lambda_{\text {irr }}^{4} \oplus$ fund $^{\oplus 16} \sim\left(\Lambda_{\text {irr }}^{2}\right)^{\oplus 5}$ \\
$\mathfrak{s p}(4)$ &
\end{tabular}

which are the same as for $\left(\Lambda^{2}\right)^{\oplus(m-4)}$. Similarly, for $\mathfrak{s p}(n), n \geq 3$, the representation $\rho_{n}=\Lambda_{\text {irr }}^{3} \oplus$ (fund) $)^{\oplus\left(2 n^{2}-7 n+6\right)}$ has invariants

$$
\begin{aligned}
\operatorname{Tr}_{\rho_{n}} F^{2}= & \left(\left(2 n^{2}-5 n+2\right)+\left(2 n^{2}-7 n+6\right)\right) \operatorname{tr} F^{2} \\
= & (2 n-4)(2 n-2) \operatorname{tr} F^{2} \\
\operatorname{Tr}_{\rho_{n}} F^{4}= & ((6 n-12)+0)\left(\operatorname{tr} F^{2}\right)^{2}+\left(\left(2 n^{2}-17 n+26\right)\right. \\
& \left.+\left(2 n^{2}-7 n+6\right)\right) \operatorname{tr} F^{4} \\
= & 3(2 n-4)\left(\operatorname{tr} F^{2}\right)^{2}+(2 n-4)(2 n-8) \operatorname{tr} F^{4}
\end{aligned}
$$

which are the same as for $\left(\Lambda_{\text {irr }}^{2}\right)^{\oplus(2 n-4)}$. We summarize these equivalences for low values of $m$ and $n$ in table 3 , as well as some analogous equivalences for $\Lambda^{4}$.

There is also a useful quartic (or biquadratic) Casimir operator when $\mathfrak{g}=\mathfrak{g}_{L} \oplus \mathfrak{g}_{R}$ is the direct sum of two simple non-abelian Lie algebras, namely, the product $\operatorname{tr}_{\mathfrak{g}_{L}}\left(F_{\mathfrak{g}_{L}}^{2}\right) \operatorname{tr}_{\mathfrak{g}_{R}}\left(F_{\mathfrak{g}_{R}}^{2}\right)$ of the normalized traces. This can be compared with other possible biquadratic Casimir operators (determined from representations) in the following way. Let $\rho$ be a representation of $\mathfrak{g}_{L} \oplus \mathfrak{g}_{R}$. If $\rho$ is irreducible, then there exist irreducible representations $\rho_{L}$ of $\mathfrak{g}_{L}$ and $\rho_{R}$ of $\mathfrak{g}_{R}$ such that $\rho=\rho_{L} \otimes \rho_{R}$. In this case, we define the representationmultiplicity of $\mathfrak{g}_{L}$ and $\mathfrak{g}_{R}$ at $\rho$ to be

$$
\mu_{\rho}\left(\mathfrak{g}_{L}, \mathfrak{g}_{R}\right)=\frac{\operatorname{Tr}_{\rho_{L}}\left(F_{\mathfrak{g}_{L}}^{2}\right) \operatorname{Tr}_{\rho_{R}}\left(F_{\mathfrak{g}_{R}}^{2}\right)}{\operatorname{tr}_{\mathfrak{g}_{L}}\left(F_{\mathfrak{g}_{L}}^{2}\right) \operatorname{tr}_{\mathfrak{g}_{R}}\left(F_{\mathfrak{g}_{R}}^{2}\right)} .
$$

By the way we defined the normalized traces for $\mathfrak{g}_{L}$ and $\mathfrak{g}_{R}, \mu_{\rho}\left(\mathfrak{g}_{L}, \mathfrak{g}_{R}\right)$ is always a non-negative integer. It is zero if one of $\rho_{L}$ and $\rho_{R}$ is trivial.

Still assuming that $\rho$ is irreducible, we can write a formula for $\mu_{\rho}\left(\mathfrak{g}_{L}, \mathfrak{g}_{R}\right)$ directly in terms of $\rho$, using the fact that $\operatorname{Tr}_{\rho_{L} \otimes \rho_{R}}\left(F_{\mathfrak{g}_{L}}^{2}\right)=\operatorname{dim}\left(\rho_{R}\right) \operatorname{Tr}_{\rho_{L}}\left(F_{\mathfrak{g}_{L}}^{2}\right)$ 
and the fact that $\operatorname{dim}(\rho)=\operatorname{dim}\left(\rho_{L}\right) \operatorname{dim}\left(\rho_{R}\right)$. The formula is:

$$
\mu_{\rho}\left(\mathfrak{g}_{L}, \mathfrak{g}_{R}\right)=\frac{1}{\operatorname{dim} \rho} \frac{\operatorname{Tr}_{\rho}\left(F_{\mathfrak{g}_{L}}^{2}\right) \operatorname{Tr}_{\rho}\left(F_{\mathfrak{g}_{R}}^{2}\right)}{\operatorname{tr}_{\mathfrak{g}_{L}}\left(F_{\mathfrak{g}_{L}}^{2}\right) \operatorname{tr}_{\mathfrak{g}_{R}}\left(F_{\mathfrak{g}_{R}}^{2}\right)}
$$

We extend this definition linearly, to arbitrary representations of $\mathfrak{g}_{L} \oplus \mathfrak{g}_{R}$, and also to the representation ring with $\mathbb{Q}$-coefficients $R\left(\mathfrak{g}_{L} \oplus \mathfrak{g}_{R}\right) \otimes \mathbb{Q}$.

Note that formula (1.2) scales linearly for multiples of an irreducible representation $\rho$ : for $\rho^{\oplus k}$, each trace in the numerator is multiplied by $k$, and the dimension in the denominator is also multiplied by $k$, giving an overall scaling by $k$.

\section{The gauge algebra}

We consider a non-singular elliptically fibered complex manifold $\pi: X \rightarrow B$, where $B$ is smooth, and with a section; we denote by $E$ the general elliptic fiber of $\pi$. Without loss of generality, we can assume that $\pi: X \rightarrow B$ is a resolution of singularities of a Weierstrass model $W \rightarrow B[29,30]$. $W$ can be described by a Weierstrass equation that locally can be written as:

$$
y^{2}=x^{3}+f x+g,
$$

where $f$ and $g$ are sections of appropriate line bundles ${ }^{3} \mathcal{O}_{B}(4 L)$ and $\mathcal{O}_{B}(6 L)$, respectively, on the base $B$. We denote by $\Delta \subset B$ the ramification locus of $\pi$; $\Delta$ has codimension one, and it is defined by the equation $4 f^{3}+27 g^{2}$ (using the standard conventions of the F-theory literature).

We are primarily interested in the case in which $X$ is a Calabi-Yau manifold; this happens when $L=-K_{B}$ is the anticanonical bundle on the base $B$. Since much of our analysis can be formulated without making this assumption, we shall do so, and only assume $L=-K_{B}$ when strictly necessary.

We also assume that the fibration $X \rightarrow B$ is equidimensional. It is known in the Calabi-Yau case [31] that at least for a base of dimension two, any Weierstrass fibration can be partially desingularized to a Calabi-Yau variety with only $\mathbb{Q}$-factorial terminal singularities ${ }^{4}$ in such a way that the elliptic

\footnotetext{
${ }^{3}$ In fact, $\mathcal{O}_{B}(L)$ is the relative dualizing sheaf of the fibration, and can be identified with the (extended) Hodge bundle.

${ }^{4}$ Terminal singularities, which include the familiar ordinary double points in dimension three occurring on conifolds, are a natural class of singularities in birational geometry. See for example $[19,32]$ in the physics literature.
} 
fibration of the Calabi-Yau variety is equidimensional. ${ }^{5}$ We are assuming here that this equidimensional Calabi-Yau variety is actually non-singular, which is a non-trivial assumption. In fact, there are $\mathbb{Q}$-factorial terminal singularities, which do not admit any (locally) Calabi-Yau desingularization, but these do not have a direct physical interpretation.

In addition, we assume that at the generic point $P$ of each codimension two subvariety $\Gamma$ of $B$, if we restrict the fibration to a general local curve $C$ passing through $P$ and transverse to each component of the discriminant containing $\Gamma$, then it is a minimal Weierstrass fibration. ${ }^{6}$ Non-minimal cases correspond to extremal transition points in the moduli space [33], and involve light strings in the spectrum. They can be analyzed from the point of view of either of the two branches of the moduli space, which are coming together at the extremal transition point. As a consequence, we do not lose anything essential by excluding them from the present analysis.

A few remarks are in order: the existence of a section is a mild assumption. In fact, if $X$ is an elliptic fibration without a section, we can consider the associated Jacobian fibration (with section) $\pi_{\mathcal{J}}: \mathcal{J}(X) \rightarrow B$, where $\mathcal{J}(X)$ is still a Calabi-Yau threefold [34-36]. Then the only assumption is again that $\mathcal{J}(X)$ is smooth; in fact $\mathcal{J}(X)$ could in principle have terminal (and not smooth) singularities, even if $B$ is smooth; however, we do not know of any such example.

In the first four columns of table 4, we list Kodaira's classification of singular fibers in the smooth resolution of a $2 \mathrm{D}$ Weierstrass fibration. Note that if we restrict a higher-dimensional fibration to a general local curve $C$ passing through $P$ and transverse to each component of the discriminant locus containing $\Gamma$, the orders of vanishing of $f, g$ and $\Delta$ at $P$ do not necessarily determine the singular fiber in the original higher-dimensional fibration (cf. [22,23]).

Each F-theory compactification on an elliptically fibered manifold $\pi$ : $X \rightarrow B$ has an associated gauge group, which can be determined ${ }^{7}$ by

\footnotetext{
${ }^{5}$ Note that there are examples with a base of dimension three in which the fibration cannot be made equidimensional while preserving the Calabi-Yau condition on the total space $[18,19]$. The physics in these cases is not completely understood.

${ }^{6} \mathrm{~A} 2 \mathrm{D}$ Weierstrass fibration is minimal at $P$ if one of the following conditions holds: $\operatorname{ord}_{u=0}(f)<4$, or $\operatorname{ord}_{u=0}(g)<6$, or $\operatorname{ord}_{u=0}\left(4 f^{3}+27 g^{2}\right)<12$, where $P$ is locally defined by the equation $u=0$.

${ }^{7}$ For Calabi-Yau fourfolds, we are describing the gauge group in the absence of flux. This "geometric" gauge group is determined in exactly the same way as for K3 surfaces or for Calabi-Yau threefolds, although the actual gauge group may be smaller since some of the gauge symmetry may be broken by flux. We shall not discuss that aspect of those models in this paper.
} 
compactifying on a circle and analyzing the corresponding singular CalabiYau variety in M-theory. The gauge group is a compact reductive group $G$ whose component group $\pi_{0}(G)$ coincides with the Tate-Shafarevich group $\amalg_{X / B}$ of the fibration [37], whose fundamental group $\pi_{1}(G)$ coincides with the Mordell-Weil group $\mathrm{MW}(X / B)$ of the fibration [38], and whose Lie algebra $\mathfrak{g}_{X / B}$ is determined by the singular fibers in codimension one as described below.

Since the gauge algebra is a reductive Lie algebra, it can be written as the direct sum of an abelian Lie algebra and a finite number of simple Lie algebras. The abelian part is given by $\pi_{1}(G) \otimes \mathbb{R}$, and so its dimension coincides with the rank of the Mordell-Weil group. In this paper, we will only consider elliptic fibrations $\pi: X \rightarrow B$ whose Mordell-Weil group has rank 0 . We denote the gauge algebra by $\mathfrak{g}_{X / B}=\bigoplus_{\Sigma} \mathfrak{g}(\Sigma)$, where the sum is taken over the components of the discriminant locus, or simply by $\mathfrak{g}$ if the meaning if clear. There are some additional anomaly cancellation conditions which must hold when there is a non-trivial abelian part of the gauge algebra, and to simplify our discussion we will not consider those here. ${ }^{8}$

Each summand of the non-abelian part of the gauge algebra in an F-theory compactification is associated with a component $\Sigma$ of the discriminant locus, and the gauge algebra summand $\mathfrak{g}(\Sigma)$ for any given component depends on the generic type of singular fiber of the Weierstrass model along that component as well as the monodromy of the components of the exceptional divisor when the singular fibers are resolved. (We sometimes denote the summand associated with a single component by $\mathfrak{g}$ when there is no danger of confusion.) The singular fibers were classified by Kodaira [39], whereas the monodromy was determined in a systematic way by Tate [40] as part of what is called Tate's algorithm. ${ }^{9}$ Tate's algorithm has been discussed in the physics literature before [4], but the conditions that determine monodromy were not spelled out in full generality.

The Kodaira classification and Tate's algorithm only depend on the generic behavior of the elliptic fibration along a particular component $\Sigma$ of the discriminant locus, and describe the behavior of the fibration near that component. In a sufficiently small open subset of the base $B$, the discriminant component $\Sigma$ can be described as $\{z=0\}$; the relevant data for

\footnotetext{
${ }^{8}$ In general, one would need to add the abelian Lie algebra $\mathrm{MW}(X / B) \otimes \mathbb{R}$ to $\bigoplus_{\Sigma} \mathfrak{g}(\Sigma)$ in order to obtain the full gauge algebra.

${ }^{9}$ In fact, Tate was interested in a more general situation which also included elliptic fibrations over fields of characteristic $p$. Since we only need to consider characteristic 0 the algorithm we present here is a slightly simplified version of the one given by Tate.
} 
the algorithm will then be (i) the orders of vanishing along $\Sigma=\{z=0\}$ of the coefficients $f$ and $g$ in the Weierstrass equation $y^{2}=x^{3}+f x+g$ and of the discriminant $\Delta=4 f^{3}+27 g^{2}$, and (ii) the quantities

$$
\left.\frac{f}{z^{\operatorname{ord}_{\Sigma}(f)}}\right|_{z=0},\left.\quad \frac{g}{z^{\operatorname{ord}_{\Sigma}(g)}}\right|_{z=0}, \quad \text { and }\left.\quad \frac{\Delta}{z^{\operatorname{ord}_{\Sigma}(\Delta)}}\right|_{z=0},
$$

which can interpreted as generically defined meromorphic sections of appropriate line bundles on $\Sigma$.

Kodaira's classification and Tate's monodromy refinement of it are presented in table 4 . The type of singularity is determined by the orders of vanishing of $f, g$ and $\Delta$ along $\Sigma$. The monodromy is described (in cases where it is relevant) by defining a certain monodromy cover of $\Sigma$ by means of a polynomial of degree 2 or 3 in an auxiliary variable $\psi$, which is a meromorphic section of a certain line bundle over $\Sigma$; the lines bundles are displayed in table 5. The key question for determining the gauge algebra is how many irreducible components this monodromy cover has. When the polynomial has degree 2, this amounts to asking whether the discriminant of the quadratic equation (which is a local meromorphic function on $\Sigma$ ) has a square root or not. One gets a smaller gauge algebra when the square root does not exist and the monodromy cover is irreducible, and a larger gauge algebra when the square root does exist and the monodromy cover is reducible. Both algebras are listed in the final column of the table.

The defining polynomial of the monodromy cover has degree 3 only in the case of Kodaira type $I_{0}^{*}$. In that case, if the cover is irreducible, the gauge algebra is $\mathfrak{g}_{2}$; if the cover has two components, the gauge algebra is $\mathfrak{s o}(7)$; and if the cover has three components, the gauge algebra is $\mathfrak{s o}(8)$.

The final row of table 4 gives the divisibility conditions, which lead to a non-minimal Weierstrass model (see Section 2). In such a case, replacing $(x, y)$ by $\left(x z^{2}, y z^{3}\right)$ leads to a new Weierstrass equation

$$
\left(y z^{3}\right)^{2}=\left(x z^{2}\right)^{3}+\left(x z^{2}\right) f+g,
$$

or

$$
y^{2}=x^{3}+x\left(f / z^{4}\right)+\left(g / z^{6}\right)
$$

in which the coefficients $(f, g)$ have been replaced by $\left(f / z^{4}, g / z^{6}\right)$ (and $\Delta$ has been replaced with $\Delta / z^{12}$ ). One can then apply the Kodaira-Tate algorithm to the new model instead. Notice that this change affects the canonical bundle, and hence the Calabi-Yau condition on the total space. 


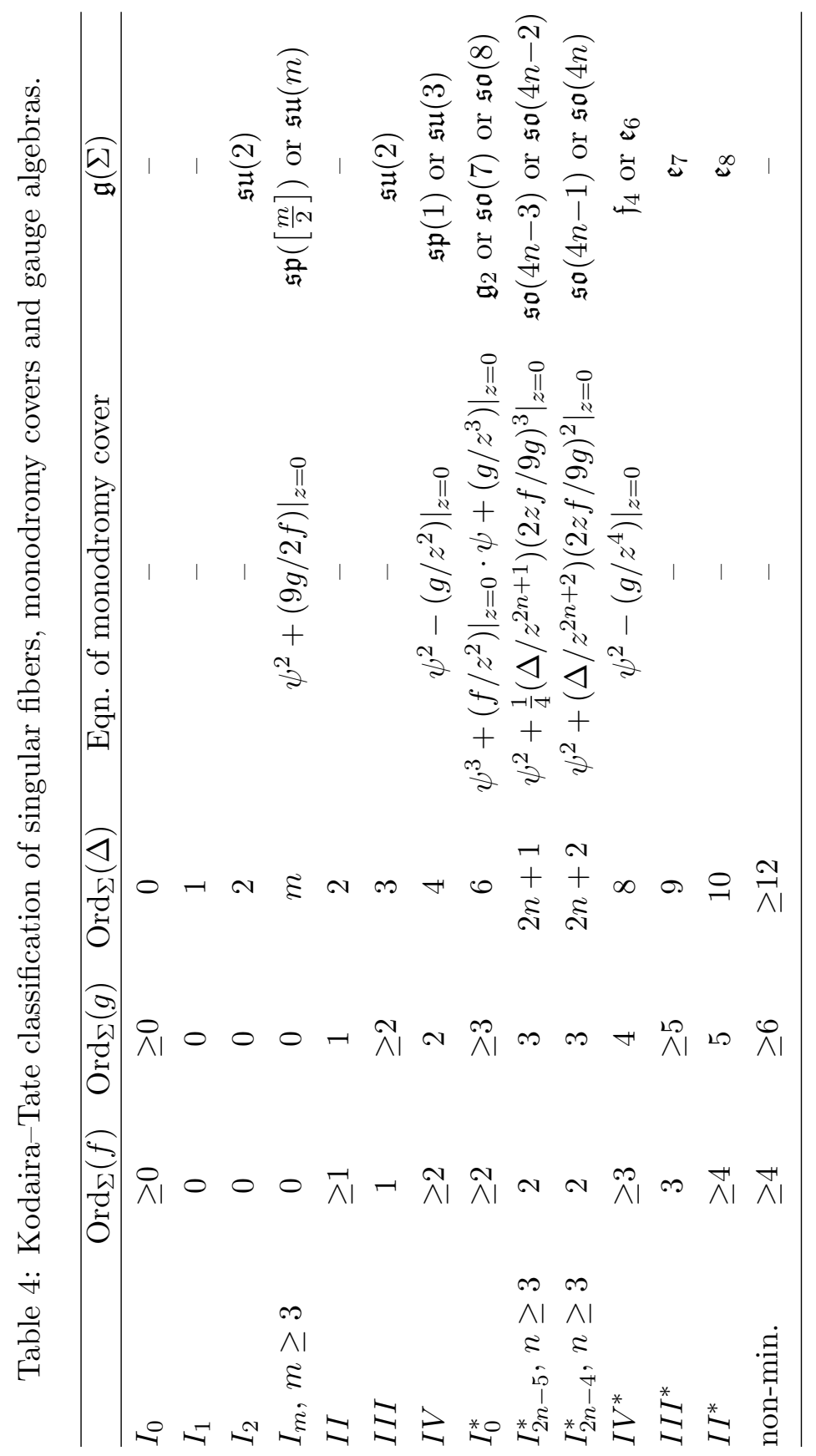


Table 5: The line bundle of which $\psi$ is a meromorphic section, in cases with a monodromy cover.

\begin{tabular}{lcccc}
\hline & $\operatorname{Ord}_{\Sigma}(f)$ & $\operatorname{Ord}_{\Sigma}(g)$ & $\operatorname{Ord}_{\Sigma}(\Delta)$ & Line bundle for $\psi$ \\
\hline$I_{m}, m \geq 3$ & 0 & 0 & $m$ & $\mathcal{O}_{\Sigma}(L)$ \\
$I V$ & $\geq 2$ & 2 & 4 & $\mathcal{O}_{\Sigma}(3 L-\Sigma)$ \\
$I_{0}^{*}$ & $\geq 2$ & $\geq 3$ & 6 & $\mathcal{O}_{\Sigma}(2 L-\Sigma)$ \\
$I_{2 n-5}^{*}, n \geq 3$ & 2 & 3 & $2 n+1$ & $\mathcal{O}_{\Sigma}(3 L-(n-1) \Sigma)$ \\
$I_{2 n-4}^{*}, n \geq 3$ & 2 & 3 & $2 n+2$ & $\mathcal{O}_{\Sigma}(4 L-n \Sigma)$ \\
$I V^{*}$ & $\geq 3$ & 4 & 8 & $\mathcal{O}_{\Sigma}(3 L-2 \Sigma)$ \\
\hline
\end{tabular}

In Appendix B, we present the detailed derivation of the information in table 4 .

In the case of $I_{m-4}^{*}$, our formulation of the monodromy condition in terms of $f, g, \Delta$ and an appropriate power of the local equation of $\Sigma$ does not seem to have been stated explicitly in the literature (even in the number theory literature, where it would also be relevant). However, it does seem to be known: one of the steps in Algorithm 7.5.1 of [41] appears to rely on this formulation.

Some aspects of the detailed geometry associated with these gauge algebras are not directly visible in table 4 . In fact, for each entry in the table, there is both a gauge algebra $\mathfrak{g}(\Sigma)$ and a covering algebra $\widetilde{\mathfrak{g}}(\Sigma)$, together with an action on $\widetilde{\mathfrak{g}}(\Sigma)$ by the monodromy group $\Gamma$ such that the fixed subspace coincides with the gauge algebra:

$$
(\widetilde{\mathfrak{g}}(\Sigma))^{\Gamma}=\mathfrak{g}(\Sigma) .
$$

The covering algebra is simply the algebra associated with the same Kodaira type but with no monodromy. As we will explain in the next section, the action of $\Gamma$ on the covering algebra preserves both the set of simple roots and the set of positive roots. The orbits of the action on the set of positive roots of the covering algebra $\widetilde{\mathfrak{g}}(\Sigma)$ coincide with the positive roots of the gauge algebra $\mathfrak{g}(\Sigma)$.

\section{The matter representation and the virtual matter cycle}

An elliptic fibration with a base of dimension at least two also has a "matter representation" (i.e., the matter representation appropriate to F-theory or M-theory), with contributions (1) from the components $\Sigma$ of the discriminant locus, (2) from the singular locus Sing $\Sigma$ of each component $\Sigma$, and (3) 
from the components $\Gamma_{\Sigma}$ of each residual discriminant locus, which is the zero-set of $\delta_{\Sigma}:=\Delta / z^{\operatorname{ord}_{\Sigma}(\Delta)}$ for any local equation $z=0$ defining $\Sigma$ (away from its singular locus). Note that the components $\Gamma_{\Sigma}$ include all pairwise intersections of components $\Sigma \cap \Sigma^{\prime}$.

If the base of the elliptic fibration has dimension two, the matter representation is a quaternionic representation of the gauge algebra $\mathfrak{g}:=\mathfrak{g}_{X / B}$. We follow the tradition of describing this matter representation by means of a complex representation $\rho: \mathfrak{g} \rightarrow \mathfrak{g l}(V)$ which determines the associated quaternionic representation as $\rho \oplus \bar{\rho}$. This traditional notation has the drawback that if an irreducible complex representation $\tau$ is itself quaternionic, there is no way to write it in the form $\rho \oplus \bar{\rho}$. We can, however, formally write it as $\frac{1}{2} \tau \oplus \frac{1}{2} \bar{\tau}$ since $\tau \cong \bar{\tau}$. Thus, the complex matter representation should actually be treated as an element of $R(\mathfrak{g}) \otimes \mathbb{Q}$, the representation ring with rational coefficients. We refer to an element $\rho \in R(\mathfrak{g}) \otimes \mathbb{Q}$ as a prequaternionic representation if $\rho \oplus \bar{\rho} \in R(\mathfrak{g})$, i.e., if $\rho \oplus \bar{\rho}$ is an actual (quaternionic) representation, not just a $\mathbb{Q}$-linear combination of representations. (One says that the corresponding 6D theory contains half-hypermultiplets in representation $\rho$.)

On the other hand, if the base of the elliptic fibration has dimension greater than two, the physical matter representation is a complex representation of $\mathfrak{g}$ rather than a quaternionic representation. The description we give here of a complex representation whose irreducible constituents are all associated with algebraic cycles of codimension two on the base is an important ingredient in the full description of the matter representation in such cases, which also involves a topologically twisted gauge theory $[42,43]$. In particular, the geometric description we give here specifies potential contributions to the matter representation, but an additional quantization problem must be solved in order to determine the multiplicity of such contributions (or even if they are present at all).

Let $\rho_{\text {matter }}$ be the complex matter representation of the gauge algebra $\mathfrak{g}$ of a $6 \mathrm{D}$ F-theory model. Since the gauge fields transform in the adjoint representation, the gauge and mixed anomalies of the theory involve the virtual representation

$$
\rho_{\text {virtual }}:=\rho_{\text {matter }}-\operatorname{adj}_{\mathfrak{g}} \in R(\mathfrak{g}) \otimes \mathbb{Q},
$$

which we call the virtual matter representation. (We work with $\mathbb{Q}$-coefficients here because of the possibility of half-hypermultiplets in $\rho_{\text {matter }}$.) This combination is also the one, which has a natural geometric interpretation. As we will show, each irreducible component of $\rho_{\text {virtual }}$ is associated with an 
algebraic cycle of codimension two. By pairing each component with the corresponding cycle, we get a virtual matter cycle

$$
Z_{\text {virtual }} \in A^{2}(B) \otimes R(\mathfrak{g}) \otimes \mathbb{Q},
$$

where $A^{2}(B)$ denotes the Chow group of codimension two cycles on the base $B$ modulo rational equivalence. ${ }^{10}$ To read off the actual matter representation in $6 \mathrm{D} \mathrm{F}$-theory, ${ }^{11}$ we just take the degree and add a copy of the adjoint representation:

$$
\rho_{\text {matter }}=\operatorname{adj}_{\mathfrak{g}} \oplus \operatorname{degree}\left(Z_{\text {virtual }}\right) .
$$

We will show in Section 7 that anomaly cancellation for these $6 \mathrm{D}$ theories follows from a relation in this "Chow group with coefficients in the representation ring of $\mathfrak{g} . "$ That relation itself, though, is not restricted to $6 \mathrm{D}$ theories, and also holds when the base of the elliptic fibration has dimension greater than two.

The matter representation for $6 \mathrm{D}$ theories gets contributions from rational curves, which are components of fibers in the Calabi-Yau resolution of a Weierstrass elliptic fibration. The deformation spaces for such rational curves can be either 1D or zero-dimensional, and Witten [44] analyzed the contribution to the matter representation in each case. We follow Witten's analysis for 1D deformation spaces, with some slight modifications in light of [9]. For the case of zero-dimensional deformation spaces, it is easier in practice to use the methods of [8] instead.

We first analyze the contributions from connected rational curves contracted by $\varphi: X \rightarrow W$ which have non-trivial deformations on $X$. Such a rational curve $C$ is rationally equivalent to a connected linear combination of components of the fiber over a general point $P \in \Sigma$ for some component $\Sigma$ of the discriminant locus. That is, $C$ corresponds to a positive root $r$ of the covering algebra $\widetilde{\mathfrak{g}}(\Sigma)$.

Now by following any closed loop on $\Sigma$, the curve $C$ may be transported to another rational curve $C^{\prime}$ : this is the monodromy action, and it comes from an action of the monodromy group $\Gamma$ on the roots of $\widetilde{\mathfrak{g}}(\Sigma)$. If the orbit of a root $r$ under the monodromy action contains $d$ elements, then the deformation space of the corresponding curve $C$ will be a connected $d$-sheeted cover of $\Sigma$.

\footnotetext{
${ }^{10} \mathrm{As}$ we will see in the construction, the algebraic cycle will itself have rational coefficients in certain cases.

${ }^{11} \mathrm{In} 4 \mathrm{D}$ F-theory models, the procedure is more complicated.
} 
Thus, if there is no monodromy, each positive root will have $\Sigma$ itself as parameter curve. By Witten's analysis, there is then a contribution of $g=g(\Sigma)$ hypermultiplets in the adjoint representation of $\mathfrak{g}(\Sigma)$ to the matter representation; here $g(\Sigma)$ is the geometric genus of $\Sigma$, that is, the genus of its normalization.

In our virtual matter representation, we subtract the vector multiplet in the adjoint representation to obtain $g-1=\frac{1}{2} \operatorname{deg}\left(\left.\left(K_{B}+\Sigma\right)\right|_{\Sigma}\right)-\frac{1}{2} \sum_{P} \mu_{P}$ $\left(\mu_{P}-1\right)$ copies of the adjoint representation, where $\mu_{P}$ are the multiplicity of all the points in $\Sigma$ including the "infinitely near points." 12 We denote by $S$ the cycle $\sum_{P} \mu_{P}\left(\mu_{P}-1\right) P$, which is supported on the singular locus of the component $\Sigma$.

The non-local contribution to the virtual matter cycle is then defined to have two parts:

$$
\left.\frac{1}{2}\left(\left.\left(K_{B}+\Sigma\right)\right|_{\Sigma}\right)-S\right) \otimes \rho_{\alpha}
$$

(where $\rho_{\alpha}$ is the adjoint representation of $\mathfrak{g}(\Sigma)$ ) represents the truly nonlocal contribution; the other part

$$
\frac{1}{2}(S) \otimes \rho_{\alpha},
$$

is actually part of the local contribution, supported at the singular locus of $\Sigma$.

If there is monodromy, then it turns out that there is an integer $d>1$ such that the positive roots of the covering algebra can be divided into two classes: the ones invariant under monodromy with parameter space $\Sigma$, and the ones not invariant under monodromy with parameter space $\widetilde{\Sigma}$ (the same parameter space for all such roots), which is a connected branched cover $\widetilde{\Sigma} \rightarrow \Sigma$ of degree $d$. Let $R$ be the ramification divisor of the branched cover, and assume for simplicity that all branching is simple. ${ }^{13}$ By the RiemannHurwitz formula, the genus $\widetilde{g}:=g(\widetilde{\Sigma})$ satisfies

$$
\widetilde{g}-1=d(g-1)+\frac{1}{2} \operatorname{deg}(R),
$$

where $g$ is the (geometric) genus of $\Sigma$. Thus,

$$
\widetilde{g}-g=(d-1)(g-1)+\frac{1}{2} \operatorname{deg}(R)
$$

\footnotetext{
${ }^{12}$ If $P \in B$ is a singular point of $\Sigma$ there is a sequence of blow ups $\phi=\varphi_{n} \circ \cdots \circ$ $\varphi_{1}: \tilde{B} \rightarrow B$ such that $\tilde{\Sigma}$ the (strict) transform of $\Sigma$ is smooth around $\phi^{-1}(P)$. The infinitely near points $P$ are the points $Q$ mapped to $P$ by $\phi$.

${ }^{13}$ It is easy to modify the analysis in the case of non-simple branching, which can only occur for $d>2$.
} 
Witten's analysis says that each invariant root contributes a space of dimension $g(\Sigma)-1$ to the virtual matter representation, and each noninvariant root contributes ${ }^{14}$ a space of dimension $g(\widetilde{\Sigma})-1$. To see how these contributions to the virtual matter representation transform under the gauge algebra, we decompose the adjoint representation of $\widetilde{\mathfrak{g}}(\Sigma)$ as a representation of $\mathfrak{g}(\Sigma)$.

In all cases, we find

$$
\operatorname{adj}_{\mathfrak{g}(\Sigma)}=\operatorname{adj}_{\mathfrak{g}(\Sigma)} \oplus \rho_{0}^{\oplus(d-1)}
$$

for some representation $\rho_{0}$ which is easily calculated (see table 6). Both kinds of roots contribute to the adjoint representation of $\mathfrak{g}(\Sigma)$, but only the non-fixed roots contribute to the representation $\rho_{0}$. Thus, this part of the virtual matter representation takes the form

$$
\begin{aligned}
(g-1) \cdot \operatorname{adj}_{\mathfrak{g}(\Sigma)}+(\widetilde{g}-g) \cdot \rho_{0}= & (g-1) \cdot \operatorname{adj}_{\mathfrak{g}(\Sigma)}+((d-1)(g-1) \\
& \left.+\frac{1}{2} \operatorname{deg}(R)\right) \cdot \rho_{0}
\end{aligned}
$$

as an element of $R(\mathfrak{g}(\Sigma)) \otimes \mathbb{Q}$. It is convenient to rewrite this as

$$
(g-1) \cdot\left(\operatorname{adj}_{\mathfrak{g}(\Sigma)}+(d-1) \cdot \rho_{0}\right)+\frac{1}{2} \operatorname{deg}(R) \cdot \rho_{0} \in R(\mathfrak{g}(\Sigma)) \otimes \mathbb{Q} .
$$

We then define one part of the non-local contribution to the virtual matter cycle to be

$$
\left.\frac{1}{2}\left(\left.\left(K_{B}+\Sigma\right)\right|_{\Sigma}\right)-S\right) \otimes \rho_{\alpha}
$$

where

$$
\rho_{\alpha}:=\operatorname{adj}_{\mathfrak{g}(\Sigma)} \oplus \rho_{0}^{\oplus(d-1)}
$$

and the other part to be

$$
R \otimes \frac{1}{2} \rho_{0}+S \otimes \frac{1}{2} \rho_{\alpha}
$$

${ }^{14}$ There is one subtlety here, in the case of $\widetilde{\mathfrak{g}}(\Sigma)=\mathfrak{s u}(2 n+1)$ and $\mathfrak{g}(\Sigma)=\mathfrak{s p}(n)$ : some of the non-invariant roots do not appear to have a vector multiplet in their spectrum. However, as argued in [9], the gauge algebra must be $\mathfrak{s p}(n)$ and this implies that the "index" contribution to the virtual matter representation must still have dimension $\widetilde{g}(\Sigma)-1$. 
Table 6: Monodromy and the representation $\rho_{0}$.

\begin{tabular}{lcccc}
\hline Type & $\mathfrak{g}(\Sigma)$ & $\widetilde{\mathfrak{g}}(\Sigma)$ & $d$ & $\rho_{0}$ \\
\hline$I_{3}$ or $I V$ & $\mathfrak{s p}(1)$ & $\mathfrak{s u}(3)$ & 2 & fund $^{\oplus 2}$ \\
$I_{2 n}, n \geq 2$ & $\mathfrak{s p}(n)$ & $\mathfrak{s u}(2 n)$ & 2 & $\Lambda_{\text {irr }}^{2}$ \\
$I_{2 n+1}, n \geq 1$ & $\mathfrak{s p}(n)$ & $\mathfrak{s u}(2 n+1)$ & 2 & $\Lambda_{\text {irr }}^{2} \oplus$ fund $^{\oplus 2}$ \\
$I_{0}^{*}$ & $\mathfrak{g}_{2}$ & $\mathfrak{s o}(8)$ & 3 & $\mathbf{7}$ \\
$I_{m-4}^{*}, m \geq 4$ & $\mathfrak{s o}(2 m-1)$ & $\mathfrak{s o}(2 m)$ & 2 & vect \\
$I V^{*}$ & $\mathfrak{f}_{4}$ & $\mathfrak{e}_{6}$ & 2 & $\mathbf{2 6}$ \\
\hline
\end{tabular}

That is, we formally represent the first term in this second part of the non-local contribution as if each point in the ramification divisor carried a representation of $\frac{1}{2} \rho_{0}$. As we will see in Section 8 , in all known cases the virtual matter representation associated with a ramification point can be written in the form $\frac{1}{2} \rho_{0}+\rho^{\prime}$, where $\rho^{\prime} \in R(\mathfrak{g})$ is a pre-quaternionic representation, often empty. Thus, this interpretation is sensible: $\rho^{\prime}$ represents the truly local matter contribution at such points.

We summarize the data about monodromy and the matter representation $\rho_{0}$ in table 6 , which shows the algebras $\mathfrak{g}(\Sigma)$ and $\tilde{\mathfrak{g}}(\Sigma)$, the degree $d$ of the monodromy cover, and the representation $\rho_{0}$ (which is easily calculated from a reference such as [45] or [46], and was already given in [10]).

In addition to the rational curves which move in families, there are isolated rational curves. If all of these curves $C_{j}$ have been calculated, then the representations they form can be determined from the intersection data $D_{i} \cdot C_{j}$ which measures the charges of these classes under the Cartan subalgebra of the gauge algebra $[5,9]$, and hence determines the weights of $\mathfrak{g}$ occurring in the representation. (Here, the $D_{i}$ are the components of the inverse images of the various components $\Sigma$.) In practice, although, the method of Katz and Vafa allows the matter representation to be determined more quickly. The equivalence of these two approaches has not been checked in every case, but see $[22,23]$ for recent progress in this direction.

Here is the Katz-Vafa method. Given a point $P \in \Sigma$ such that $\pi^{-1}(P)$ contains rational curves, which do not move in families, take a general small disc $D$ in the base, which meets $\Sigma$ at $P$, and consider the family of elliptic curves over $D$. Our key assumption (see Section 2) implies that this will never have a non-minimal Kodaira fiber; thus, there is a particular Kodaira 
fiber over $P$ on the Weierstrass model, and the inverse image of $D$ on the Weierstrass model is a surface with a rational double-point. ${ }^{15}$

Now consider a deformation of $D$, i.e., a family of disks $D_{t}$ meeting $\Sigma$ at a variable point $P_{t}$ with $D_{0}=D$. By the Brieskorn-Grothendieck theorem $[48,49]$, after an appropriate basechange $t=\tau^{\ell}$, the singularities on $\pi^{-1}\left(D_{\tau^{\ell}}\right)$ can be simultaneously resolved. Moreover, there is a versal space $V$ of deformations of $\pi^{-1}\left(D_{0}\right)$ which can be simultaneously resolved, and the parameter curve $\{\tau\}$ of our base-changed family will map to $V$. Let $m$ be the ramification of that map at the origin. Then locally, $t=s^{k}$ where $s$ is the coordinate on a local disc within $V$ and $k=\ell / m$.

The Kodaira fiber over $P=P_{0}$ determines a covering algebra $\widetilde{\mathfrak{g}}_{P}$, and the Kodaira fiber over $P_{s^{k}}$ determines a generic covering algebra $\widetilde{\mathfrak{g}}(\Sigma)$, with an embedding $\widetilde{\mathfrak{g}}(\Sigma) \subset \widetilde{\mathfrak{g}}_{P}$.

The Katz-Vafa method says: there is a complex representation $\rho_{P}$ of $\widetilde{\mathfrak{g}}(\Sigma)$ such that the adjoint representation of $\widetilde{\mathfrak{g}}_{P}$ decomposes under $\widetilde{\mathfrak{g}}(\Sigma)$ as

$$
\operatorname{adj}_{\widetilde{\mathfrak{g}}_{P}}=\operatorname{adj}_{\mathfrak{g}(\Sigma)} \oplus \rho_{P} \oplus \overline{\rho_{P}} \oplus \mathbf{1}^{\oplus\left(\operatorname{rank}\left(\widetilde{\mathfrak{g}}_{P}\right)-\operatorname{rank}(\tilde{\mathfrak{g}}(\Sigma))\right)},
$$

the corresponding local contribution to the matter representation is the charged $\operatorname{part}^{16}$ of $\frac{1}{k} \cdot\left(\left.\rho_{P}\right|_{\mathfrak{g}(\Sigma)}\right)$.

(As we will see in examples, when $P$ is a ramification point for monodromy, this representation naturally contains $\frac{1}{2} \rho_{0}$ as a summand.) As a cycle on $\Sigma$, we define the local contribution to be the charged part of

$$
\frac{1}{k} \cdot P \otimes\left(\left.\rho_{P}\right|_{\mathfrak{g}(\Sigma)}\right)
$$

Note that the representation $\left.\rho_{P}\right|_{\mathfrak{g}(\Sigma)}$ could a priori have a summand which is neutral under the gauge algebra $\mathfrak{g}(\Sigma)$; we exclude any such summands from the local contribution to the matter representation.

The Katz-Vafa method is in fact ambiguous in at least one case, as observed in [22], since there are two inequivalent embeddings of $A_{7}$ into $E_{8}[50,51]$. We will discuss this case further in Section 8.

\footnotetext{
${ }^{15}$ For clarity, we stress that here we are referring to "Kodaira type" as determined by orders of vanishing as in table 4 , and not assuming that the fiber in the nonsingular model is one of Kodaira's fibers. In fact, in codimension at least two on the base, the fiber need not coincide with Kodaira's fibers [22, 23, 47].

${ }^{16}$ Recall (see for example [10]) that the charged part is defined as follows: Let $\rho$ be a representation of a Lie algebra $\mathfrak{g}$, with Cartan subalgebra $\mathfrak{h}$. The charged dimension of $\rho$ is $(\operatorname{dim} \rho)_{\mathrm{ch}}=\operatorname{dim}(\rho)-\operatorname{dim}\left(\left.k e r \rho\right|_{\mathfrak{h}}\right)$.
} 
There are several ways to extract information from the virtual matter cycle, which will prove useful in checking anomaly cancellation. First, for any component $\Sigma$ of the discriminant locus which contributes a non-abelian summand $\mathfrak{g}(\Sigma)$ to the gauge algebra, we can restrict the matter representation or the matter cycle to $\mathfrak{g}(\Sigma)$ :

$$
\left.Z_{\text {virtual }}\right|_{\mathfrak{g}(\Sigma)}
$$

obtaining a cycle that will involve representations of $\mathfrak{g}(\Sigma)$ only.

Second, for any pair of distinct components $\Sigma \neq \Sigma^{\prime}$ of the discriminant locus, which each contribute a non-abelian summand to the gauge algebra, we can compute the representation-multiplicity of $\mathfrak{g}(\Sigma)$ and $\mathfrak{g}\left(\Sigma^{\prime}\right)$ at the cycle $Z_{\text {virtual }}$ :

$$
\mu_{Z_{\text {virtual }}}\left(\mathfrak{g}(\Sigma), \mathfrak{g}\left(\Sigma^{\prime}\right)\right)
$$

Here, we have extended the definition of representation-multiplicity in a natural way to include the case of an algebraic cycle $Z$ whose coefficients are representations, i.e., to elements of $A^{2}(B) \otimes R\left(\mathfrak{g}_{L} \otimes \mathfrak{g}_{R}\right) \otimes \mathbb{Q}$. In this extension, the representation-multiplicity $\mu_{Z}\left(\mathfrak{g}, \mathfrak{g}^{\prime}\right)$ is an ordinary cycle, i.e., a linear combination of subvarieties with numerical coefficients.

\section{The Tate cycle for $\Sigma$}

For each component $\Sigma$ of the discriminant locus corresponding to a nonabelian summand of the gauge algebra, we now introduce another cycle of codimension two which we call the Tate cycle for $\Sigma$, since its definition is closely related to Tate's algorithm. The part of the anomaly cancellation condition involving $\mathfrak{g}(\Sigma)$ will be satisfied if the restriction of the virtual matter cycle to $\mathfrak{g}(\Sigma)$ is rationally equivalent to the Tate cycle for $\Sigma$, or more generally, if the two are Casimir equivalent in degrees 2 and 4 . This formulation becomes a criterion which can be checked locally, for many specific kinds of degenerate fibers in elliptic fibrations. Sections 8 and 9 will be devoted to checking this criterion in a wide variety of cases.

Tate's algorithm, reviewed in Appendix B, starts from a Weierstrass model of an elliptic fibration and, making changes of coordinates that involve rational functions on the base as well as appropriate relative blowups, finds expressions in which it is possible to determine things such as monodromy of components of the exceptional divisor of the blowups. Here, we adapt that algorithm and produce certain rational sections of line bundles on $\Sigma$ which can be used to mimic Tate's forms of the equations. The zeros and poles of those rational sections are then associated with specific representations 
of the gauge algebra, producing an algebraic cycle of codimension two with coefficients in the representation $\operatorname{ring} R(\mathfrak{g}) \otimes \mathbb{Q}$, as we previously did with the virtual matter cycle. As we will see, it can be verified that anomaly cancellation holds whenever the virtual matter cycle is equal to the Tate cycle for all components $\Sigma$ (and a certain condition holds on intersections between pairs of components of the discriminant locus).

Our starting point is an elliptic fibration in Weierstrass form

$$
y^{2}=x^{3}+f x+g \text {. }
$$

We assume that $\Sigma$ is a component of the discriminant locus $\Delta=0$ of multiplicity $m_{\Sigma}$, and let $z=0$ be a local defining equation for $\Sigma$ around a nonsingular point of $\Sigma$. We let $\delta_{\Sigma}=\left.\left(\Delta / z^{m_{\Sigma}}\right)\right|_{\Sigma}$ be the residual discriminant, a section of $\left.\left(12 L-m_{\Sigma} \Sigma\right)\right|_{\Sigma}$.

- For Kodaira fibers of type $I_{m}$, by changing coordinates in $x$ we may assume that the singular point is located at the origin, and the equation takes the form

$$
y^{2}=x^{3}+u x^{2}+v x+w
$$

Since $\left.v\right|_{\Sigma}$ and $\left.w\right|_{\Sigma}$ both vanish, we can determine $\left.u\right|_{\Sigma}$ from $f$ and $g$ in the following way. Completing the cube in (4.1) gives

$$
\begin{aligned}
& f=-\frac{1}{3} u^{2}+v \\
& g=\frac{2}{27} u^{3}-\frac{1}{3} u v+w
\end{aligned}
$$

and so $\left(\left.u\right|_{\Sigma}\right)^{2}=-\left.3 f\right|_{\Sigma}$ and $\left(\left.u\right|_{\Sigma}\right)^{3}=\left.\frac{27}{2} g\right|_{\Sigma}$. Taking the ratio, it follows that $\left.u\right|_{\Sigma}=\left.(-9 g / 2 f)\right|_{\Sigma}$, which can be regarded as a non-zero rational section of $\left.2 L\right|_{\Sigma}$. Note that since this is expressed in terms of $f$ and $g$, it is independent of the choice of coordinates used to obtain (4.1).

We now define a rational section $\gamma_{\Sigma}$ of $\left.\left(8 L-m_{\Sigma} \Sigma\right)\right|_{\Sigma}$ by

$$
\gamma_{\Sigma}:=\frac{\delta_{\Sigma}}{\left(\left.u\right|_{\Sigma}\right)^{2}} .
$$

When $m>2$, Tate's analysis shows that the ramification divisor for monodromy is $\operatorname{div}\left(\left.u\right|_{\Sigma}\right)$. In this case, we also define $\beta_{\Sigma}:=\left.u\right|_{\Sigma}$, and note that there is a natural algebraic cycle on $\Sigma$ which we can identify with $\frac{1}{2} \operatorname{div}\left(\beta_{\Sigma}\right)$, rationally equivalent to $\left.L\right|_{\Sigma}$.

- For Kodaira fibers of type $I I, I I I$ or $I V$, all of the vanishing of $\delta_{\Sigma}$ is attributable to either vanishing of $\left(f / z^{k}\right) \mid \Sigma$ or of $\left.\left(g / z^{k}\right)\right|_{\Sigma}$; we call this 
$\gamma_{\Sigma}$ in types $I I$ and $I I I$ when there is no possibility of monodromy, and $\beta_{\Sigma}$ in type $I V$ when Tate's analysis tells us that $\operatorname{div}\left(\left.\left(g / z^{2}\right)\right|_{\Sigma}\right)$ is the ramification divisor for monodromy. Note that since singular fibers of type $I_{1}$ and $I I$ are irreducible on the non-singular model, they make no contribution to the gauge algebra. For this reason, those types are not included in tables 7 to 9 .

- For Kodaira fibers of type $I_{m-4}^{*}$, there is an auxiliary equation

$$
y^{2}=x^{3}+\left(f / z^{2}\right) x+\left(g / z^{3}\right)
$$

describing part of a relevant blowup. The cubic equation in $x$ has no a priori factorization when $m-4=0$, and this is the trickiest case to characterize: the characterization depends on how many irreducible factors the right-hand side of (4.3) has after restriction to $\Sigma$. (We do not define either $\beta_{\Sigma}$ or $\gamma_{\Sigma}$ in this case.) If the right-hand side of (4.3) restricted to $\Sigma$ has a linear factor

$$
\left.\left(x^{3}+\left(f / z^{2}\right) x+\left(g / z^{3}\right)\right)\right|_{\Sigma}=(x-a)\left(x^{2}+d x+e\right),
$$

then $a$ and $d$ are rational sections of $\left.(2 L-\Sigma)\right|_{\Sigma}$, while $e$ is a rational section of $\left.(4 L-2 \Sigma)\right|_{\Sigma}$. On the other hand, if the right-hand side of (4.3) restricted to $\Sigma$ has three linear factors

$$
\left.\left(x^{3}+\left(f / z^{2}\right) x+\left(g / z^{3}\right)\right)\right|_{\Sigma}=(x-a)(x-b)(x-c)
$$

then $a, b$ and $c$ are all rational sections of $\left.(2 L-\Sigma)\right|_{\Sigma}$.

For $I_{m-4}^{*}$ with $m-4>0$, the auxiliary Equation (4.3) has one double root and one simple root, and by changing coordinates we can put the double root at the origin, giving a new auxiliary equation of the form

$$
y^{2}=x^{3}+(u / z) x^{2}+\left(v / z^{2}\right) x+\left(w / z^{3}\right)
$$

with $z \mid v / z^{2}$ and $z \mid w / z^{3}$. We let $\gamma_{\Sigma}=\left.(u / z)\right|_{\Sigma}=\left.(-9 g / 2 z f)\right|_{\Sigma}$, a rational section of $\left.(2 L-\Sigma)\right|_{\Sigma}$, determined analogously to the $I_{m}$ case by completing the cube to obtain $f$ and $g$ from $u, v$ and $w$. Tate's algorithm for $I_{m-4}^{*}$ is quite involved, but the upshot is that $\delta_{\Sigma}=(-1)^{k+1} \beta_{\Sigma}$ $\gamma_{\Sigma}^{k}$ for some rational section $\beta_{\Sigma}$ of $((12-2 k) L-(m+2-k) \Sigma)$, where $k=3$ when $m$ is odd, and $k=2$ when $m$ is even. (This is explained in 
Table 7: Main construction.

\begin{tabular}{lccc}
\hline Type & $\beta_{\Sigma}$ & $\gamma_{\Sigma}$ & $\delta_{\Sigma}$ \\
\hline$I_{2}$ & - & $\left.\delta_{\Sigma}\left(4 f^{2} / 81 g^{2}\right)\right|_{\Sigma}$ & $\left.\gamma_{\Sigma}(-9 g / 2 f)^{2}\right|_{\Sigma}$ \\
$I_{m}, m \geq 3$ & $\left.(-9 g / 2 f)\right|_{\Sigma}$ & $\delta_{\Sigma} / \beta_{\Sigma}^{2}$ & $\beta_{\Sigma}^{2} \gamma_{\Sigma}$ \\
$I I I$ & - & $\left.(f / z)\right|_{\Sigma}$ & $4 \gamma_{\Sigma}^{3}$ \\
$I V$ & $\left.\left(g / z^{2}\right)\right|_{\Sigma}$ & - & $27 \beta_{\Sigma}^{2}$ \\
$I_{0}^{*}$ & & Special case & \\
$I_{2 n-5}^{*}, n \geq 3$ & $\delta_{\Sigma} / \gamma_{\Sigma}^{3}$ & $\left.(-9 g / 2 z f)\right|_{\Sigma}$ & $\beta_{\Sigma} \gamma_{\Sigma}^{3}$ \\
$I_{2 n-4}^{*}, n \geq 3$ & $-\delta_{\Sigma} / \gamma_{\Sigma}^{2}$ & $\left.(-9 g / 2 z f)\right|_{\Sigma}$ & $-\beta_{\Sigma} \gamma_{\Sigma}^{2}$ \\
$I V^{*}$ & $\left.\left(g / z^{4}\right)\right|_{\Sigma}$ & - & $27 \beta_{\Sigma}^{2}$ \\
$I I I^{*}$ & - & $\left.\left(f / z^{3}\right)\right|_{\Sigma}$ & $4 \gamma_{\Sigma}^{3}$ \\
$I I^{*}$ & - & $\left.\left(g / z^{5}\right)\right|_{\Sigma}$ & $27 \gamma_{\Sigma}^{2}$ \\
\hline & & &
\end{tabular}

detail in Appendix B.) The key fact about $\beta_{\Sigma}$ is that $\operatorname{div}\left(\beta_{\Sigma}\right)$ is the ramification divisor for monodromy. ${ }^{17}$

- For Kodaira fibers of type $I V^{*}, I I I^{*}$ or $I I^{*}$, once again all of the vanishing of $\delta_{\Sigma}$ is attributable to either vanishing of $\left.\left(f / z^{k}\right)\right|_{\Sigma}$ or of $\left.\left(g / z^{k}\right)\right|_{\Sigma}$; we call this $\gamma_{\Sigma}$ in types $I I I^{*}$ and $I I^{*}$ when there is no possibility of monodromy, and $\beta_{\Sigma}$ in type $I V^{*}$ when Tate's analysis tells us that $\operatorname{div}\left(\left.\left(g / z^{4}\right)\right|_{\Sigma}\right)$ is the ramification divisor for monodromy.

The definitions of $\beta_{\Sigma}$ and $\gamma_{\Sigma}$ are summarized in table 7 , which also shows how $\delta_{\Sigma}$ is related to these. Note that in some cases, only one of $\beta_{\Sigma}$ and $\gamma_{\Sigma}$ is defined, and in the case of $I_{0}^{*}$, neither one is defined (and there is correspondingly no description of $\delta_{\Sigma}$ ). Whenever $\beta_{\Sigma}$ is defined, it describes the ramification of a double cover of $\Sigma$. Thus, either $\sqrt{\beta_{\Sigma}}$ is well-defined on $\Sigma$ (when the double cover splits), or $\sqrt{\beta_{\Sigma}}$ is well-defined on the double cover; in either case, $\frac{1}{2} \operatorname{div}\left(\beta_{\Sigma}\right)$ is a well-defined algebraic cycle class on $\Sigma$. The equivalence classes of this cycle ${ }^{18}$ and the other cycles $\operatorname{div}\left(\gamma_{\Sigma}\right)$ and $\operatorname{div}\left(\delta_{\Sigma}\right)$ are displayed in table 8 .

One key thing to note: by our assumptions, we cannot have a point at which the multiplicities of $(f, g, \Delta)$ exceed $(4,6,12)$. This implies that (1) for $I_{m-4}^{*}, m-4 \geq 4, \gamma_{\Sigma}$ may not vanish, and (2) for $I I^{*}, \gamma_{\Sigma}$ may not

\footnotetext{
${ }^{17}$ This corrects a statement from [10], where the ramification divisor was misidentified for $I_{m-4}^{*}$.

${ }^{18}$ Since $\Sigma$ is a divisor on $B$, any divisor on $\Sigma$ is an algebraic cycle of codimension two on $B$.
} 
Table 8: The constituents of the Tate cycle.

\begin{tabular}{lccc}
\hline Type & $\frac{1}{2} \operatorname{div}\left(\beta_{\Sigma}\right)$ & $\operatorname{div}\left(\gamma_{\Sigma}\right)$ & $\operatorname{div}\left(\delta_{\Sigma}\right)$ \\
\hline$I_{2}$ & - & $\left.(8 L-2 \Sigma)\right|_{\Sigma}$ & $\left.(12 L-2 \Sigma)\right|_{\Sigma}$ \\
$I_{m}, m \geq 3$ & $\left.L\right|_{\Sigma}$ & $\left.(8 L-m \Sigma)\right|_{\Sigma}$ & $\left.(12 L-m \Sigma)\right|_{\Sigma}$ \\
$I I I$ & - & $\left.(4 L-\Sigma)\right|_{\Sigma}$ & $\left.(12 L-3 \Sigma)\right|_{\Sigma}$ \\
$I V$ & $\left.(3 L-\Sigma)\right|_{\Sigma}$ & - & $\left.(12 L-4 \Sigma)\right|_{\Sigma}$ \\
$I_{0}^{*}$ & \multicolumn{2}{c}{ special case } & $\left.(12 L-6 \Sigma)\right|_{\Sigma}$ \\
$I_{2 n-5}^{*}, n \geq 3$ & $\left.(3 L-(n-1) \Sigma)\right|_{\Sigma}$ & $\left.(2 L-\Sigma)\right|_{\Sigma}$ & $\left.(12 L-(2 n+1) \Sigma)\right|_{\Sigma}$ \\
$I_{2 n-4}^{*}, n \geq 3$ & $\left.(4 L-n \Sigma)\right|_{\Sigma}$ & $\left.(2 L-\Sigma)\right|_{\Sigma}$ & $\left.(12 L-(2 n+2) \Sigma)\right|_{\Sigma}$ \\
$I V^{*}$ & $\left.(3 L-2 \Sigma)\right|_{\Sigma}$ & - & $\left.(12 L-8 \Sigma)\right|_{\Sigma}$ \\
$I I I^{*}$ & - & $\left.(4 L-3 \Sigma)\right|_{\Sigma}$ & $\left.(12 L-9 \Sigma)\right|_{\Sigma}$ \\
$I I^{*}$ & - & $\left.(6 L-5 \Sigma)\right|_{\Sigma}$ & $\left.(12 L-10 \Sigma)\right|_{\Sigma}$ \\
\hline
\end{tabular}

vanish. Thus, for $I_{m-4}^{*}, m-4 \geq 4,\left.(2 L-\Sigma)\right|_{\Sigma}$ must be trivial, and for $I I^{*}$, $\left.(6 L-5 \Sigma)\right|_{\Sigma}$ must be trivial.

For each gauge algebra, we associate a representation (with $\mathbb{Q}$ coefficients) to each of these cycles, as specified in ${ }^{19}$ table 9 . (Note that we do not assign a representation to the excluded cases: there is no $\rho_{\gamma}$ for either $I_{m-4}^{*}$, $m-4 \geq 4$, or $I I^{*}$.) The case of $I_{0}^{*}$ is not completely described in the Table, but follows our earlier discussion in the various cases. First, if the cubic equation is irreducible (so that the gauge algebra is $\mathfrak{g}_{2}$ ), then each point of the ramification locus of the cover is associated with $1 / 2$ of a $7 \mathrm{D}$ representation. Second, if the cubic equation has one linear and one quadratic factor (so that the gauge algebra is $\mathfrak{s o}(7)$ ), the linear factor determines a bundle equivalent to $\frac{1}{6} \operatorname{div}\left(\delta_{\Sigma}\right)$, and the zeros of that are identified with the spinor representation, while the ramification points of the quadratic factor correspond to the vector representation. And third, if the cubic equation factors completely, then each factor determines a divisor equivalent to $\frac{1}{6} \operatorname{div}\left(\delta_{\Sigma}\right)$, and the zeros of each of those correspond to one of the $8 \mathrm{D}$ representations of $\mathfrak{s o}(8)$.

We now define the Tate cycle for $\Sigma$ to be

$$
Z_{\text {Tate }, \Sigma}=\left.\frac{1}{2}(-L+\Sigma)\right|_{\Sigma} \otimes \rho_{\alpha}+\frac{1}{2} \operatorname{div}\left(\beta_{\Sigma}\right) \otimes \rho_{\sqrt{\beta}}+\operatorname{div}\left(\gamma_{\Sigma}\right) \otimes \rho_{\gamma} .
$$

\footnotetext{
${ }^{19}$ These representations have not been chosen arbitrarily. Rather, as we will see in Section 7, they are precisely the representations we need in order to cancel anomalies.
} 
Table 9: The Tate representations $($ in $R(\mathfrak{g}(\Sigma)) \otimes \mathbb{Q})$.

\begin{tabular}{|c|c|c|c|c|}
\hline Type & $\mathfrak{g}(\Sigma)$ & $\rho_{\alpha}$ & $\rho_{\sqrt{\beta}}$ & $\rho_{\gamma}$ \\
\hline$I_{2}$ & $\mathfrak{s u}(2)$ & $\operatorname{adj}$ & - & fund \\
\hline$I_{3}$ & $\mathfrak{s p}(1)$ & $\operatorname{adj}+2 \cdot$ fund & fund & fund \\
\hline$I_{3}$ & $\mathfrak{s u}(3)$ & $\operatorname{adj}$ & fund & fund \\
\hline $\begin{array}{l}I_{2 n}, \\
n \geq 2\end{array}$ & $\mathfrak{s p}(n)$ & $\operatorname{adj}+\Lambda_{\mathrm{irr}}^{2}$ & $\Lambda_{\text {irr }}^{2}$ & fund \\
\hline $\begin{array}{l}I_{2 n+1} \\
n \geq 1\end{array}$ & $\mathfrak{s p}(n)$ & $\begin{array}{l}\text { adj }+\Lambda_{\text {irr }}^{2} \\
+2 \cdot \text { fund }\end{array}$ & $\Lambda_{\text {irr }}^{2}+$ fund & fund \\
\hline $\begin{array}{l}I_{m} \\
m \geq 2\end{array}$ & $\mathfrak{s u}(m)$ & adj & $\Lambda^{2}$ & fund \\
\hline$I I I$ & $\mathfrak{s u}(2)$ & $\operatorname{adj}$ & - & $2 \cdot$ fund \\
\hline$I V$ & $\mathfrak{s p}(1)$ & $\operatorname{adj}+2 \cdot$ fund & $3 \cdot$ fund & - \\
\hline$I V$ & $\mathfrak{s u}(3)$ & $\operatorname{adj}$ & $3 \cdot$ fund & - \\
\hline$I_{0}^{*}$ & $\mathfrak{g}_{2}$ & $\operatorname{adj}+2 \cdot 7$ & \multicolumn{2}{|c|}{$\mathbf{7} \mapsto \frac{1}{2} \operatorname{div}\left(\delta_{\Sigma}\right)$} \\
\hline$I_{0}^{*}$ & $\mathfrak{s o}(7)$ & $\operatorname{adj}+$ vect & \multicolumn{2}{|c|}{$\operatorname{spin} \mapsto \frac{1}{6} \operatorname{div}\left(\delta_{\Sigma}\right)$, vect $\mapsto \frac{1}{3} \operatorname{div}\left(\delta_{\Sigma}\right)$} \\
\hline$I_{0}^{*}$ & $\mathfrak{s o}(8)$ & $\operatorname{adj}$ & \multicolumn{2}{|c|}{ vect, $\operatorname{spin}_{+}, \operatorname{spin}_{-} \mapsto \frac{1}{6} \operatorname{div}\left(\delta_{\Sigma}\right)$} \\
\hline$I_{1}^{*}$ & $\mathfrak{s o}(9)$ & $\operatorname{adj}+$ vect & vect & $\operatorname{spin}_{*}$ \\
\hline$I_{1}^{*}$ & $\mathfrak{s o}(10)$ & $\operatorname{adj}$ & vect & $\operatorname{spin}_{*}$ \\
\hline$I_{2}^{*}$ & $\mathfrak{s o}(11)$ & $\operatorname{adj}+$ vect & vect & $\frac{1}{2} \cdot \operatorname{spin}_{*}$ \\
\hline$I_{2}^{*}$ & $\mathfrak{s o}(12)$ & $\operatorname{adj}$ & vect & $\frac{1}{2} \cdot \operatorname{spin}_{*}$ \\
\hline$I_{3}^{*}$ & $\mathfrak{s o}(13)$ & $\operatorname{adj}+$ vect & vect & $\frac{1}{4} \cdot \operatorname{spin}_{*}+$ vect \\
\hline$I_{3}^{*}$ & $\mathfrak{s o}(14)$ & $\operatorname{adj}$ & vect & $\frac{1}{4} \cdot \operatorname{spin}_{*}+$ vect \\
\hline $\begin{array}{l}I_{m-4}^{*} \\
m \geq 8\end{array}$ & $\mathfrak{s o}(2 m-1)$ & $\operatorname{adj}+$ vect & vect & $\mathrm{N} / \mathrm{A}$ \\
\hline $\begin{array}{l}I_{m-4}^{*} \\
m \geq 8\end{array}$ & $\mathfrak{s o}(2 m)$ & $\operatorname{adj}$ & vect & $\mathrm{N} / \mathrm{A}$ \\
\hline$I V^{*}$ & $\mathfrak{f}_{4}$ & $\operatorname{adj}+\mathbf{2 6}$ & 26 & - \\
\hline$I V^{*}$ & $\mathfrak{e}_{6}$ & $\operatorname{adj}$ & 27 & - \\
\hline$I I I^{*}$ & $\mathfrak{e}_{7}$ & $\operatorname{adj}$ & - & $\frac{1}{2} \cdot 56$ \\
\hline$I I^{*}$ & $\mathfrak{e}_{8}$ & $\operatorname{adj}$ & - & $\mathrm{N} / \mathrm{A}$ \\
\hline
\end{tabular}

Aside from the first term, this cycle has a representative (with $\mathbb{Q}$-coefficients) which is localized at the zeros of $\delta_{\Sigma}$. Each component $\Gamma_{\Sigma}$ of $\left\{\delta_{\Sigma}=0\right\}$ thus has an associated representation, namely, its coefficient in $Z_{\text {Tate, } \Sigma}$.

A very simple manipulation with tables 8 and 9 , collecting terms by irreducible representation instead of by irreducible cycle, now shows that 
Table 10: Cycles associated with representations.

\begin{tabular}{|c|c|c|}
\hline$\underline{\mathfrak{g}(\Sigma)}$ & $\rho$ & $\Gamma_{\rho}$ \\
\hline $\mathfrak{s u}(2)$ & $\begin{array}{l}\text { adj } \\
\text { fund }\end{array}$ & $\begin{array}{l}\left.\frac{1}{2}(-L+\Sigma)\right|_{\Sigma} \\
\left.(8 L-2 \Sigma)\right|_{\Sigma}\end{array}$ \\
\hline $\mathfrak{s u}(3)$ & $\begin{array}{l}\text { adj } \\
\text { fund }\end{array}$ & $\begin{array}{l}\left.\frac{1}{2}(-L+\Sigma)\right|_{\Sigma} \\
\left.(9 L-3 \Sigma)\right|_{\Sigma}\end{array}$ \\
\hline $\begin{array}{l}\mathfrak{s u}(m) \\
m \geq 4\end{array}$ & $\begin{array}{l}\text { adj } \\
\text { fund } \\
\Lambda^{2}\end{array}$ & $\begin{array}{c}\left.\frac{1}{2}(-L+\Sigma)\right|_{\Sigma} \\
\left.(8 L-m \Sigma)\right|_{\Sigma} \\
\left.L\right|_{\Sigma}\end{array}$ \\
\hline $\begin{array}{l}\mathfrak{s p}(n), \\
n \geq 2\end{array}$ & $\begin{array}{l}\text { adj } \\
\text { fund } \\
\Lambda_{\text {irr }}^{2}\end{array}$ & $\begin{array}{l}\left.\frac{1}{2}(-L+\Sigma)\right|_{\Sigma} \\
\left.(8 L-2 n \Sigma)\right|_{\Sigma} \\
\left.\quad \frac{1}{2}(L+\Sigma)\right|_{\Sigma}\end{array}$ \\
\hline $\begin{array}{l}\mathfrak{s o}(\ell) \\
7 \leq \ell \leq 14\end{array}$ & $\begin{array}{c}\text { adj } \\
\text { vect } \\
\operatorname{spin}_{*}\end{array}$ & $\begin{array}{c}\left.\frac{1}{2}(-L+\Sigma)\right|_{\Sigma} \\
\left.\frac{1}{2}((\ell-4) L+(6-\ell) \Sigma)\right|_{\Sigma} \\
\left.\frac{1}{\operatorname{dim}\left(\operatorname{spin}_{*}\right)}(32 L-16 \Sigma)\right|_{\Sigma}\end{array}$ \\
\hline $\begin{array}{l}\mathfrak{s o}(4 n-1) \\
n \geq 4\end{array}$ & $\begin{array}{c}\text { adj } \\
\text { vect }\end{array}$ & $\begin{array}{c}\left.\frac{1}{2}(-L+\Sigma)\right|_{\Sigma} \\
\left.\left(\frac{7}{2} L-\left(n-\frac{1}{2}\right) \Sigma\right)\right|_{\Sigma}\end{array}$ \\
\hline $\begin{array}{l}\mathfrak{s o}(4 n) \\
n \geq 4\end{array}$ & $\begin{array}{c}\text { adj } \\
\text { vect }\end{array}$ & $\begin{array}{l}\left.\frac{1}{2}(-L+\Sigma)\right|_{\Sigma} \\
\left.(4 L-n \Sigma)\right|_{\Sigma}\end{array}$ \\
\hline $\begin{array}{l}\mathfrak{s o}(4 n+1) \\
n \geq 4\end{array}$ & $\begin{array}{l}\text { adj } \\
\text { vect }\end{array}$ & $\begin{array}{c}\left.\frac{1}{2}(-L+\Sigma)\right|_{\Sigma} \\
\left.\left(\frac{5}{2} L-\left(n-\frac{1}{2}\right) \Sigma\right)\right|_{\Sigma}\end{array}$ \\
\hline $\begin{array}{l}\mathfrak{s o}(4 n+2), \\
n \geq 4\end{array}$ & $\begin{array}{l}\text { adj } \\
\text { vect }\end{array}$ & $\begin{array}{l}\left.\frac{1}{2}(-L+\Sigma)\right|_{\Sigma} \\
\left.(3 L-n \Sigma)\right|_{\Sigma}\end{array}$ \\
\hline $\mathfrak{e}_{6}$ & $\begin{array}{l}\operatorname{adj} \\
27\end{array}$ & $\begin{array}{l}\left.\frac{1}{2}(-L+\Sigma)\right|_{\Sigma} \\
\left.(3 L-2 \Sigma)\right|_{\Sigma}\end{array}$ \\
\hline $\mathfrak{e}_{7}$ & $\begin{array}{l}\operatorname{adj} \\
56\end{array}$ & $\begin{array}{l}\left.\frac{1}{2}(-L+\Sigma)\right|_{\Sigma} \\
\left.\frac{1}{2}(4 L-3 \Sigma)\right|_{\Sigma}\end{array}$ \\
\hline $\begin{array}{l}\mathfrak{e}_{8} \\
\mathfrak{f}_{4}\end{array}$ & $\begin{array}{l}\operatorname{adj} \\
\operatorname{adj}\end{array}$ & $\begin{array}{l}\left.\frac{1}{2}(-L+\Sigma)\right|_{\Sigma} \\
\left.\frac{1}{2}(-L+\Sigma)\right|_{\Sigma}\end{array}$ \\
\hline $\mathfrak{g}_{2}$ & $\begin{array}{c}26 \\
\operatorname{adj} \\
7\end{array}$ & $\begin{array}{l}\left.\frac{1}{2}(5 L-3 \Sigma)\right|_{\Sigma} \\
\left.\frac{1}{2}(-L+\Sigma)\right|_{\Sigma} \\
\left.(5 L-2 \Sigma)\right|_{\Sigma}\end{array}$ \\
\hline
\end{tabular}


the Tate cycle is rationally equivalent to a cycle of the form

$$
Z_{\text {Tate }, \Sigma} \sim \sum_{\rho} \Gamma_{\rho} \otimes \rho
$$

where the cycle classes $\Gamma_{\rho}$ are given in table 10, and remarkably, depend only on the gauge algebra $\mathfrak{g}(\Sigma)$, not on its particular geometric realization.

Let us compare the "Tate representations" of this paper with the representations described in [10, table A]. There are some minor differences, but for the most part the representation denoted $\rho_{0}$ in the previous paper corresponds to the representation $\rho_{0}$ from (3.1) and also coincides with the new $\rho_{\sqrt{\beta}}$ when there is monodromy; the representation $\rho_{1}$ in the earlier paper corresponds to $\rho_{\sqrt{\beta}}$ in this paper when there is no monodromy; and the representation $\rho_{2}$ in the earlier paper corresponds to $\rho_{\gamma}$ in this paper.

\section{Six-dimensional anomalies}

The anomaly of a supersymmetric 6D theory (with no abelian local factor in its gauge group) consists of a pure gravitational anomaly which is a quartic Casimir in the gravitational curvature, a pure gauge anomaly that is a quartic Casimir in the gauge curvature, and a mixed anomaly, which is a product of quadratic Casimirs in the gravitational and gauge curvatures [27,52]. Each of these anomalies must vanish.

Without a Green-Schwarz term, such a theory is typically anomalous. The total anomaly (in a suitable normalization [20]) is:

$$
\kappa \cdot A \cdot \operatorname{tr} R^{4}+B \cdot\left(\operatorname{tr} R^{2}\right)^{2}+\frac{1}{6} \operatorname{tr} R^{2} \sum X_{i}^{(2)}-\frac{2}{3} \sum X_{i}^{(4)}+4 \sum_{i<j} Y_{i j}
$$

where $\kappa$ is a non-zero proportionality constant, $A=\left(n_{V}-n_{H}+273-29 n_{T}\right)$ and $B=\left(\frac{9-n_{T}}{8}\right)$. Here $n_{\mathrm{T}}$ is the number of tensor multiplets, $n_{\mathrm{V}}$ the number of vector multiplets, $n_{H}$ the number of hypermultiplets, ${ }^{20}$ and

$$
\begin{aligned}
X_{i}^{(n)} & =\operatorname{Tr}_{\mathrm{adj}} F_{i}^{n}-\sum_{\rho} n_{\rho} \operatorname{Tr}_{\rho} F_{i}^{n}, \\
Y_{i j} & =\sum_{\rho, \sigma} n_{\rho \sigma} \operatorname{Tr}_{\rho} F_{i}^{2} \operatorname{Tr}_{\sigma} F_{j}^{2}
\end{aligned}
$$

where $R$ is the curvature of the Levi-Civita connection, and $F$ is the curvature of the gauge connection. In these formulas, $\operatorname{Tr}_{\text {adj }}$ means the trace in

\footnotetext{
${ }^{20}$ The geometric interpretation of these various multiplets is given in Section 6 below.
} 
the adjoint representation, $\operatorname{Tr}_{\rho}$ denotes the trace in the representation $\rho$ of the simple algebra $\mathfrak{g}_{i}$ (see Appendix A), $n_{\rho}$ is the multiplicity of the representation $\rho$ of $\mathfrak{g}_{i}$ in the matter representation, ${ }^{21}$ and $n_{\rho \sigma}$ is the multiplicity of the representation $\rho \otimes \sigma$ of $\mathfrak{g}_{i} \oplus \mathfrak{g}_{j}$ in the matter representation.

We can rewrite these expressions in terms of the total virtual representation

$$
\rho_{\text {virtual }}=-\operatorname{adj}_{\mathfrak{g}}+\sum_{\rho} n_{\rho} \cdot \rho
$$

as follows. First, ${ }^{22}$ note that

$$
Y_{i j}=\mu_{\rho_{\text {virtual }}}\left(\mathfrak{g}_{i}, \mathfrak{g}_{j}\right) \operatorname{tr}_{\mathfrak{g}_{i}}\left(F_{i}^{2}\right) \operatorname{tr}_{\mathfrak{g}_{j}}\left(F_{j}^{2}\right)
$$

that is, $Y_{i j}$ can be expressed in terms of the representation-multiplicity. Next, we define

$$
\begin{aligned}
X^{(n)}\left(\rho_{\text {virtual }}\right) & =-\sum X_{i}^{(n)}, \\
Y\left(\rho_{\text {virtual }}\right) & =\sum_{i \neq j} Y_{i j}=2 \sum_{i<j} Y_{i j} .
\end{aligned}
$$

(The awkward sign in the first definition is due to the sign in the original definition of $X_{i}^{(n)}$, and is designed so that $X^{(n)}\left(\sum_{\rho} n_{\rho} \cdot \rho\right)=\sum_{i, \rho} n_{\rho} \operatorname{Tr}_{\rho} F_{i}^{n}$.) This lets us rewrite the anomaly in the form

$$
\begin{aligned}
\kappa & \cdot A \cdot \operatorname{tr} R^{4}+B \cdot\left(\operatorname{tr} R^{2}\right)^{2}-\frac{1}{6} \operatorname{tr} R^{2} X^{(2)}\left(\rho_{\text {virtual }}\right) \\
& +\frac{2}{3} X^{(4)}\left(\rho_{\text {virtual }}\right)+2 Y\left(\rho_{\text {virtual }}\right) .
\end{aligned}
$$

To ensure an anomaly free 6D theory, a term of Green-Schwarz type must be included $[20,53,54]$. In the Calabi-Yau threefold case, Sadov [55] derived the form of the Green-Schwarz term by reducing from ten dimensions, for all F-theory models, which in type IIB language admit only D7-branes and orientifold O7-planes. (In the Kodaira language used in this paper, this corresponds to allowing only singular fibers of types $I_{m}$ and $I_{m-4}^{*}$.) This was extended to the general case in [10], as we review here.

\footnotetext{
${ }^{21}$ In the physics literature one says that there are " $n_{\rho}$ hypermultiplets in the representation $\rho$."

${ }^{22}$ Note that since no irreducible component of the "adjoint" term in $\rho_{\text {virtual }}$ is charged under two different non-abelian summands of $\mathfrak{g}$, this term has no effect on $Y_{i j}$.
} 
The Green-Schwarz term in the action takes the form

$$
\int \Psi \cdot\left(\frac{1}{2} K_{B} \otimes \operatorname{tr} R^{2}+2 \sum_{\Sigma}\left(\Sigma \otimes \operatorname{tr}_{\mathfrak{g}(\Sigma)} F^{2}\right)\right),
$$

where $\Psi$ is a two-form field in the effective $6 \mathrm{D}$ theory labeled by an element of $H^{2}(B, Z)$ (with indices suppressed) obtained by dimensional reduction from a four-form field in ten dimensions, and $\cdot$ denotes the intersection product in $H^{2}(B, Z)$. The key expression

$$
D_{\text {gauge }}(F):=\sum_{\Sigma}\left(\Sigma \otimes \operatorname{tr}_{\mathfrak{g}(\Sigma)} F^{2}\right)
$$

which we call the gauge divisor, involves the normalized trace for the corresponding summand of the Lie algebra; that this normalization gives the correct linear combination was verified by Sadov for $\mathfrak{g}(\Sigma)=\mathfrak{u}(m), \mathfrak{g}(\Sigma)=\mathfrak{s p}(m)$ and $\mathfrak{g}(\Sigma)=\mathfrak{s o}(\ell)$. The normalization for exceptional groups is a consequence of the study of anomalies made in [10].

More generally, for an elliptic fibration that may not be Calabi-Yau, we propose as Green-Schwarz term

$$
\int \Psi \cdot\left(-\frac{1}{2} L \otimes \operatorname{tr} R^{2}+2 \sum_{\Sigma}\left(\Sigma \otimes \operatorname{tr}_{\mathfrak{g}(\Sigma)} F^{2}\right)\right)
$$

where $L$ is the line bundle used to construct the Weierstrass model (which coincides with $-K_{B}$ in the Calabi-Yau threefold case).

Including such a term in the action (as we must, in deriving F-theory from the type IIB string), there is a contribution to the anomaly of

$$
\begin{aligned}
-\frac{1}{2} & \left(-\frac{1}{2} L \otimes \operatorname{tr} R^{2}+2 D_{\text {gauge }}(F)\right)^{2} \\
= & -\frac{1}{8} L^{2} \otimes\left(\operatorname{tr} R^{2}\right)^{2}+\sum_{\Sigma}(L \cdot \Sigma) \otimes\left(\operatorname{tr} R^{2} \operatorname{tr}_{\mathfrak{g}(\Sigma)} F^{2}\right) \\
& -2\left(\sum_{\Sigma} \Sigma \otimes\left(\operatorname{tr}_{\mathfrak{g}(\Sigma)} F^{2}\right)\right)^{2} .
\end{aligned}
$$

To obtain an anomaly free theory, then, requires four conditions:

$$
n_{\mathrm{V}}-n_{H}+273-29 n_{\mathrm{T}}=0
$$




$$
\begin{aligned}
\frac{9-n_{\mathrm{T}}}{8} & =\frac{1}{8} L^{2} \\
\frac{1}{6} X^{(2)}\left(\rho_{\text {virtual }}\right) & =\sum(L \cdot \Sigma) \operatorname{tr}_{\mathfrak{g}(\Sigma)} F^{2} \\
& =L \cdot D_{\text {gauge }}(F) \\
\frac{1}{3} X^{(4)}\left(\rho_{\text {virtual }}\right)+Y\left(\rho_{\text {virtual }}\right) & =\left(\sum_{\Sigma} \Sigma \otimes\left(\operatorname{tr}_{\mathfrak{g}(\Sigma)} F^{2}\right)\right)^{2} \\
& =D_{\text {gauge }}(F)^{2}
\end{aligned}
$$

Note that:

- Condition (5.3) is equivalent to a formula for the topological Euler characteristic of the total space of the elliptic fibration, this was the focus of [10] (see Section 6).

- Condition (5.4) in the Calabi-Yau case is equivalent to $K_{B}^{2}=9-n_{\mathrm{T}}$, and follows from the fact that $n_{\mathrm{T}}$ is the number of times $\mathbb{P}^{2}$ must be blown up (or one fewer than the number of times a Hirzebruch surface $\mathbb{F}_{n}$ must be blown up) to obtain $B$ (see Section 6 ).

- Conditions (5.5) and (5.6) put specific constraints on the matter representation of the theory.

Our main result is a stronger form of the last two conditions, which are described in Section 7.

\section{The Euler characteristic}

It is known [33] that if $X$ is Calabi-Yau the geometric interpretation of the numbers of multiplets is

$$
\begin{aligned}
& n_{\mathrm{V}}=\operatorname{dim}(G), \\
& n_{\mathrm{T}}=h^{1,1}(B)-1, \\
& n_{\mathrm{H}}=H_{\mathrm{ch}}+H_{0}, \text { with } H_{0}=h^{2,1}(X)+1,
\end{aligned}
$$

where $n_{\mathrm{V}}$ and $n_{\mathrm{T}}$ denote the number of vector, tensor multiplets, respectively; $n_{\mathrm{H}}$ is the number of hypermultiplets and $H_{\mathrm{ch}}$ denotes the charged hypermultiplets and $H_{0}$ denotes the neutral hypermultiplets.

Moreover, since we are assuming $\operatorname{rk} M W(X / B)=0$, we have

$$
\operatorname{rk}(G)=h^{1,1}(X)-h^{1,1}(B)-1 .
$$


In [10] we provided an algorithm to analyze $H_{\mathrm{ch}}$ in terms of the topological Euler characteristic, but we do not derive explicitly this formula from the physics. We shall do that here.

Translated into geometric quantities, the key formula 5.3 becomes

$$
h^{2,1}(X)+1+H_{\mathrm{ch}}-\operatorname{dim}(G)=273-29 n_{\mathrm{T}}
$$

and

$$
\frac{1}{2} \chi_{\mathrm{top}}(X)=\operatorname{rk}(G)+n_{\mathrm{T}}+2-h^{2,1}(X) .
$$

The first statement follows from Equation (6.1); the second holds because $X$ is a Calabi-Yau threefold. In fact the equality $h^{1,0}(X)=h^{2,0}(X)=0$ and $h^{3,0}(X)=1$ imply:

$$
\begin{aligned}
\chi_{\mathrm{top}}(X) & =b_{0}-b_{1}+b_{2}-b_{3}+b_{4} \\
& =1-0+h^{1,1}(X)-\left(2+2 h^{2,1}(X)\right)+h^{1,1}(X)-0+1 \\
& =2 h^{1,1}(X)-2 h^{2,1}(X) .
\end{aligned}
$$

Thus, using Equation (6.4), we find

$$
\frac{1}{2} \chi_{\mathrm{top}}(X)=h^{1,1}(X)-h^{2,1}(X)=2+n_{\mathrm{T}}+\operatorname{rk}(G)-h^{2,1}(X) .
$$

To interpret formula (6.5) as a formula for the Euler characteristic, we need a few more geometric facts.

Let $\pi: X \rightarrow B$ be an elliptic Calabi-Yau threefold; then

$$
\begin{array}{r}
\chi_{\mathrm{top}}(B)=2+h^{1,1}(B), \\
10=h^{1,1}(B)+K_{B}^{2}, \\
9=n_{\mathrm{T}}+K_{B}^{2} .
\end{array}
$$

Our assumptions in fact imply that $B$ is either rational or an Enriques surface [56]: then $\chi\left(\mathcal{O}_{B}\right)=1$. The second equality follows from Noether's formula $K_{B}^{2}+\chi_{\text {top }}(B)=12 \chi\left(\mathcal{O}_{B}\right)$ and the third from Equation (6.1). Now if we add Equation (6.9) with (6.6) we find

$$
\frac{1}{2} \chi_{\mathrm{top}}(X)+30 K_{B}^{2}=270-30 n_{\mathrm{T}}+2+n_{\mathrm{T}}+\operatorname{rk}(G)-h^{2,1}(X),
$$

from which the equation

$$
\frac{1}{2} \chi_{\mathrm{top}}(X)+30 K_{B}^{2}=273-29 n_{\mathrm{T}}+\operatorname{rk}(G)-\left(h^{2,1}(X)+1\right) .
$$


immediately follows. Then the condition 5.3 is then equivalent to

$$
\frac{1}{2} \chi_{\mathrm{top}}(X)+30 K_{B}^{2}=H_{\mathrm{ch}}-(\operatorname{dim}(G)-\operatorname{rk}(G)) .
$$

In [10] we defined and analyzed the quantity $\mathcal{R}:=\frac{1}{2} \chi_{\text {top }}(X)+30 K_{B}^{2}$ in terms of the matter representation.

Remark 6.1. In general, if $n_{\mathrm{T}}=h^{1,1}(B)-1$, then $9-n_{\mathrm{T}}=K_{B}^{2}$ implies that either $h^{1,0}(B)=h^{2,0}(B)=0$ or $h^{1,0}(B)=5$ and $h^{2,0}(B)=4$. There are other type of surfaces, which satisfy these hypothesis, most notably some of general type.

\section{Anomaly cancellation in the Chow group}

It turns out that the last two conditions for anomaly cancellation hold not only numerically, but as actual algebraic cycles. That is, if we use the virtual matter cycle $Z_{\text {virtual }}$ on the left-hand side, and interpret the intersection on the right-hand side as intersection in the Chow group, then we get the stronger statements

$$
\frac{1}{3} X^{(4)}\left(Z_{\text {virtual }}\right)+Y\left(Z_{\text {virtual }}\right)=\left(\sum_{\Sigma} \Sigma \otimes\left(\operatorname{tr}_{\mathfrak{g}(\Sigma)} F^{2}\right)\right)^{2}=D_{\text {gauge }}(F)^{2},
$$

which are to be interpreted as equality of codimension two cycles on the base $B$ up to rational equivalence.

The coefficients in these cycles are Casimir operators for the gauge algebra $\mathfrak{g}$; for these relations to be satisfied, they must hold in each sector of the algebra of Casimir operators. In particular, the first statement must hold as an equality of quadratic Casimir operators when restricted to each summand $\mathfrak{g}(\Sigma)$ :

$$
X^{(2)}\left(\left.Z_{\text {virtual }}\right|_{\mathfrak{g}(\Sigma)}\right)=6(L \cdot \Sigma) \operatorname{tr}_{\mathfrak{g}(\Sigma)} F^{2}
$$

the second statement must hold as an equality of quartic Casimir operators, which restricted to each summand $\mathfrak{g}(\Sigma)$ :

$$
X^{(4)}\left(Z_{\text {virtual }} \mid \mathfrak{g}(\Sigma)\right)=3(\Sigma \cdot \Sigma)\left(\operatorname{tr}_{\mathfrak{g}(\Sigma)} F^{2}\right)^{2} ;
$$


and the second statement must also hold as an equality of bi-quadratic Casimir operators when restricted to each pair of summands $\mathfrak{g}(\Sigma) \oplus \mathfrak{g}\left(\Sigma^{\prime}\right)$ :

$$
\mu_{Z_{\text {virtual }}}\left(\mathfrak{g}(\Sigma), \mathfrak{g}\left(\Sigma^{\prime}\right)\right)=\Sigma \cdot \Sigma^{\prime}
$$

(In the last equation, we suppressed the generator $\operatorname{tr}_{\mathfrak{g}(\Sigma)}\left(F^{2}\right) \operatorname{tr}_{\mathfrak{g}\left(\Sigma^{\prime}\right)}\left(F^{2}\right)$ of the bi-quadratic Casimirs since that is taken care of in the definition of the representation-multiplicity $\mu$.)

On the other hand, it is straightforward to verify using tables 2 and 10 that

$$
\begin{aligned}
& X^{(2)}\left(Z_{\text {Tate }, \Sigma}\right)=\left.6 L\right|_{\Sigma} \otimes \operatorname{tr}_{\mathfrak{g}(\Sigma)} F^{2} \\
& X^{(4)}\left(Z_{\text {Tate }, \Sigma}\right)=\left.3 \Sigma\right|_{\Sigma} \otimes\left(\operatorname{tr}_{\mathfrak{g}(\Sigma)} F^{2}\right)^{2}
\end{aligned}
$$

(and this in fact motivated our definition of the Tate cycles). Thus, our main anomaly cancellation result is:

Main result. The elliptic fibration defines an anomaly free theory if it satisfies (5.3) and (5.4), if there is a Casimir equivalence in degrees 2 and 4

$$
\left.Z_{\text {virtual }}\right|_{\mathfrak{g}(\Sigma)} \sim Z_{\text {Tate }, \Sigma}
$$

for all $\Sigma$, and if there is a rational equivalence of cycles

$$
\mu_{Z_{\text {virtual }}}\left(\mathfrak{g}(\Sigma), \mathfrak{g}\left(\Sigma^{\prime}\right)\right)=\Sigma \cdot \Sigma^{\prime}
$$

for all $\Sigma \neq \Sigma^{\prime}$.

In the Calabi-Yau case, when $L=-K_{B}$, the non-local part of these equations holds, since the contribution of the adjoint representation is $g-1$, which is calculated by the cycle $\left.\frac{1}{2}\left(K_{B}+\Sigma\right)\right|_{\Sigma}$. Thus, the only things to check are the local contributions (including copies of $\rho_{\alpha}$ associated with singularities of $\Sigma$ ), and these can be checked cycle-by-cycle.

Local anomaly cancellation. Suppose that $X$ is Calabi-Yau. Let $\Gamma$ be a subvariety of codimension two, let $\rho_{\Gamma}$ be the local contribution to the matter representation associated with $\Gamma$, and let $\Sigma_{1}, \ldots, \Sigma_{k}$ be the components of the discriminant locus which pass through $\Gamma$ and which contribute non-abelian summands to the gauge algebra. If $\rho_{\Gamma} \mid \Sigma_{j}$ is Casimir equivalent in degrees 2 and 4 to the contribution at $\Gamma$ to the Tate representation for $\Sigma_{j}$, and if for 
all $i \neq j$

$$
\mu_{\rho_{\Gamma}}\left(\mathfrak{g}\left(\Sigma_{i}\right), \mathfrak{g}\left(\Sigma_{j}\right)\right)=\operatorname{mult}_{\Gamma}\left(\Sigma_{i}, \Sigma_{j}\right),
$$

that is, the representation-multiplicity coincides with the intersection multiplicity, then local anomaly cancellation holds at $\Gamma$.

Note that the local contribution to the Tate representation for $\Sigma$ is easily calculated from the Weierstrass equation, since it depends only on the order of zero or pole along $\Gamma$ of $\beta_{\Sigma}$ and/or $\gamma_{\Sigma}$, as well as the genus drop at a singular point of $\Sigma .^{23}$

Anomaly cancellation is thus reduced to this kind of local computation. We carry it out for a wide variety of examples in the next section.

\section{Examples}

We have seen that the anomaly cancellation can be reduced to a straightforward property about codimension two cycles on which the elliptic fibration structure degenerates. Namely, given such a cycle and a component $\Sigma$ of the discriminant locus containing the cycle, one can calculate the local contribution $^{24}$ to the Tate cycle for $\Sigma$ directly from the Weierstrass equation; one can also calculate intersection multiplicities of all pairs of components of the discriminant locus, which pass through the cycle. This data must then be compared with the contribution of that cycle to the virtual matter representation.

In this section and the next, we carry out this verification for the standard "generic" codimension two singularities of elliptic fibrations from [4] (as already verified in [10]) as well as for some new codimension two singularities such as the one from [21]. We also introduce some singularities, which are considered here for the first time. All of our examples are local, considered in a neighborhood of a particular codimension two locus defined by $\{z=t=0\}$.

Let us first consider cases in which the Kodaira type along $\Sigma=\{z=0\}$ is $I_{m}$ with $m=2 n$ or $m=2 n+1$. The generalized Weierstrass forms proposed

\footnotetext{
${ }^{23}$ The simplest example of this phenomenon is an ordinary double point of $\Sigma$ in the $I_{m}$ case, which Sadov argued [55] is associated with the symmetric representation $S^{2} V$ of $\mathfrak{s u}(m)$. Since $S^{2} V$ is Casimir equivalent to adj $-\Lambda^{2}$, this is accounted for by an appropriate local computation.

${ }^{24}$ We have already verified the anomaly cancellation condition for the global contributions to the Tate and virtual matter cycles, so we can now focus on local contributions only.
} 
in $[4,21]$ can all be written in the general form (see [21, Appendix A])

$$
y^{2}=x^{3}+a_{2} x^{2}+a_{4, n} z^{n} x+a_{6,2 n} z^{2 n},
$$

with additional restrictions for various particular cases. The discriminant for such a Weierstrass form is

$$
\Delta=4 a_{2}^{3} a_{6,2 n} z^{2 n}-a_{2}^{2} a_{4, n}^{2} z^{2 n}+O\left(z^{3 n}\right),
$$

whenever $n \geq 1$.

Example 8.1. Consider $I_{m}, m \geq 3$, with $a_{2} \neq 0$ at the codimension two singular point, and let $n=[m / 2]$. Then we can rewrite (8.1) as

$$
y^{2}=x^{3}+a_{2}\left(x+\frac{a_{4,[m / 2]}}{2 a_{2}} z^{[m / 2]}\right)^{2}+t z^{m}+O\left(z^{m+1}\right),
$$

which yields a discriminant that satisfies

$$
\Delta=4 a_{2}^{3} t z^{m}+O\left(z^{m+1}\right) .
$$

Thus, we have identified the local contribution to the residual discriminant with the codimension two locus $\{z=t=0\}$. Note that when the coefficients are generic, the Kodaira fiber along a disc through $z=t=0$ has type $I_{m+1}$.

Since the coefficients of $x^{1}$ and $x^{0}$ in (8.3) are divisible by $z, \beta_{\Sigma}$ coincides with the coefficient of $x^{2}$, i.e., $\beta_{\Sigma}=a_{2}$. Since $\delta_{\Sigma}=4 a_{2}^{3} t$, it follows that $\gamma_{\Sigma}=4 a_{2} t$.

Example 8.2. Consider $I_{2 n}, n \geq 2$, in the form (8.1) with $a_{2}=t$ vanishing at the codimension two singular point. Thus, we have equation

$$
y^{2}=x^{3}+t x^{2}+a_{4, n} z^{n} x+a_{6,2 n} z^{2 n},
$$

and discriminant

$$
\Delta=t^{2}\left(4 t a_{6,2 n}-a_{4, n}^{2}\right) z^{2 n}+O\left(z^{2 n+2}\right) .
$$

The higher order of vanishing of the error term is important, because it shows that the total order of vanishing of $\Delta$ in the limit is at least $2 n+2$. Thus, the special fiber is $I_{2 n-4}^{*}$. 
Again $\beta_{\Sigma}$ is the coefficient of $x^{2}$, i.e., $\beta_{\Sigma}=t$; since $\delta_{\Sigma}=t^{2}\left(4 t a_{6,2 n}-a_{4, n}^{2}\right)$ it follows that $\gamma_{\Sigma}=4 t a_{6,2 n}-a_{4, n}^{2}$.

We now specialize to cases in which the Kodaira type along $\Sigma$ is $I_{2 n+1}$, $n \geq 1$. The new generalized Weierstrass form in this case proposed in [21] (with a minor change of notation) is

$$
\begin{aligned}
y^{2}= & x^{3}+\left(\frac{1}{4} \mu \nu^{2}+a_{2,1} z\right) x^{2}+\left(\frac{1}{2} \mu \nu \xi+a_{4, n+1} z\right) z^{n} x \\
& +\left(\frac{1}{4} \mu \xi^{2}+a_{6,2 n+1} z\right) z^{2 n}
\end{aligned}
$$

and the discriminant takes the form

$$
\begin{gathered}
\left(\frac{1}{4} \mu \nu^{2}+a_{2,1} z\right)^{2}\left[\mu\left(\xi^{2} a_{2,1}-\nu \xi a_{4, n+1}+\nu^{2} a_{6,2 n+1}\right)\right. \\
\left.+\left(4 a_{2,1} a_{6,2 n+1}-a_{4, n+1}^{2}\right) z\right] z^{2 n+1}+O\left(z^{3 n}\right) .
\end{gathered}
$$

Example 8.3. Consider $I_{2 n+1}, n \geq 4$ in the form (8.7) with $\mu=t$ vanishing at the singular point. The equation becomes

$$
y^{2}=x^{3}+\left(\frac{1}{4} t \nu^{2}+a_{2,1} z\right) x^{2}+\left(\frac{1}{2} t \nu \xi+a_{4, n+1} z\right) z^{n} x+\left(! \frac{1}{4} t \xi^{2}+a_{6,2 n+1} z\right) z^{2 n}
$$

and the discriminant is

$$
\begin{aligned}
& \left(\frac{1}{4} t \nu^{2}+a_{2,1} z\right)^{2}\left[t\left(\xi^{2} a_{2,1}-\nu \xi a_{4, n+1}+\nu^{2} a_{6,2 n+1}\right)\right. \\
& \left.+\left(4 a_{2,1} a_{6,2 n+1}-a_{4, n+1}^{2}\right) z\right] z^{2 n+1}+O\left(z^{2 n+4}\right)
\end{aligned}
$$

since $3 n \geq 2 n+4$. The order of vanishing increases by at least 3 at $z=t=0$.

By construction, $\beta_{\Sigma}=\frac{1}{4} t \nu^{2} ;$ since

$$
\delta_{\Sigma}=\left(\frac{1}{4} t \nu^{2}+a_{2,1} z\right)^{2} t\left(\xi^{2} a_{2,1}-\nu \xi a_{4, n+1}+\nu^{2} a_{6,2 n+1}\right)
$$

it follows that $\gamma_{\Sigma}=t\left(\xi^{2} a_{2,1}-\nu \xi a_{4, n+1}+\nu^{2} a_{6,2 n+1}\right)$. 
Example 8.4. We again consider $I_{2 n+1}, n \geq 3$, using Equation (8.7), this time with $\nu=t$ vanishing at the singular point. The equation becomes

$$
\begin{aligned}
y^{2}= & x^{3}+\left(\frac{1}{4} \mu t^{2}+a_{2,1} z\right) x^{2}+\left(\frac{1}{2} \mu t \xi+a_{4, n+1} z\right) z^{n} x \\
& +\left(\frac{1}{4} \mu \xi^{2}+a_{6,2 n+1} z\right) z^{2 n}
\end{aligned}
$$

and the discriminant takes the form

$$
\begin{gathered}
\left(\frac{1}{4} \mu t^{2}+a_{2,1} z\right)^{2}\left[\mu\left(\xi^{2} a_{2,1}-t \xi a_{4, n+1}+t^{2} a_{6,2 n+1}\right)\right. \\
\left.+\left(4 a_{2,1} a_{6,2 n+1}-a_{4, n+1}^{2}\right) z\right] z^{2 n+1}+O\left(z^{2 n+3}\right)
\end{gathered}
$$

since $3 n \geq 2 n+3$. The order of vanishing increases by at least 2 for this example, and we have $\beta_{\Sigma}=\frac{1}{4} \mu t^{2}, \gamma_{\Sigma}=\mu\left(\xi^{2} a_{2,1}-t \xi a_{4, n+1}+t^{2} a_{6,2 n+1}\right)$.

Note that Example 4 includes cases without monodromy, in the generalized Weierstrass form from [4]:

$$
y^{2}+a_{1} x y+a_{3, n} z^{n} y=x^{3}+a_{2,1} z x^{2}+a_{4, n+1} z^{n+1} x+a_{6,2 n+1},
$$

since, after completing the square, we see that this is the same as Equation (8.7), with $\mu=1, \nu=a_{1}$ and $\xi=a_{3, n}$.

Example 8.5. Consider $I_{m-4}^{*}$ with $m \geq 7$. The generalized Weierstrass form from [4] can be written as

$$
y^{2}=x^{3}+a_{2,1} z x^{2}+a_{4,[(m+1) / 2]} z^{[(m+1) / 2]} x+a_{6, m-1} z^{m-1},
$$

with discriminant

$$
\Delta=4 a_{2,1}^{3} a_{6, m-1} z^{m+2}-a_{2,1}^{2} a_{4,[(m+1) / 2]} z^{2+2[(m+1) / 2]}+O\left(z^{m+3}\right) .
$$

For our example, we assume that $a_{2,1}$ does not vanish at $z=t=0$. Then we can rewrite the equation in the form

$$
y^{2}=x^{3}+a_{2,1} z\left(x+\frac{a_{4,[(m+1) / 2]}}{2 a_{2,1}} z^{[(m-1) / 2]}\right)^{2}+t z^{m-1}+O\left(z^{m}\right),
$$

where we have set $a_{6, m-1}=t+\left(a_{4,[(m+1) / 2]}^{2} / 4 a_{2,1}\right) z^{2-m+2[(m-1) / 2]}$. It follows that the discriminant takes the form

$$
\Delta=4 a_{2,1}^{3} t z^{m+2}+O\left(z^{m+3}\right)
$$


Table 11: First group of examples. In $\# 5, \alpha=1$ if $m$ is odd and $\alpha=-a_{2,1}$ if $m$ is even.

\begin{tabular}{lcllllll}
\hline & Gen. & Spec. & Eqn. & Disc. & $\beta_{\Sigma}$ & $\gamma_{\Sigma}$ & $t$ \\
\hline 1 & $I_{m}, m \geq 4$ & $I_{m+1}$ & $(8.3)$ & $(8.4)$ & $a_{2}$ & $4 a_{2} t$ & $s$ \\
2 & $I_{2 n}, n \geq 2$ & $I_{2 n-4}^{*}$ & $(8.5)$ & $(8.6)$ & $t$ & $4 t a_{6,2 n}-a_{4, n}^{2}$ & $s^{2}$ \\
3 & $I_{2 n+1}, n \geq 4$ & $I_{2 n-2}^{*}$ & $(8.9)$ & $(8.10)$ & $\frac{1}{4} t \nu^{2}$ & $t\left(\xi^{2} a_{2,1}-\nu \xi a_{4, n+1}\right.$ & $s^{2}$ \\
& & & & & & $\left.+\nu^{2} a_{6,2 n+1}\right)$ & \\
4 & $I_{2 n+1}, n \geq 3$ & $I_{2 n-3}^{*}$ & $(8.11)$ & $(8.12)$ & $\frac{1}{4} \mu t^{2}$ & $\mu\left(\xi^{2} a_{2,1}-t \xi a_{4, n+1}\right.$ & $s$ \\
& & & & & & $\left.+t^{2} a_{6,2 n+1}\right)$ & $s^{2}$ \\
5 & $I_{m-4}^{*}, m \geq 7$ & $I_{m-3}^{*}$ & $(8.13)$ & $(8.14)$ & $4 \alpha t$ & 1 &
\end{tabular}

Since the coefficients of $x^{1}$ and $x^{0}$ in (8.15) are divisible by $z^{3}$ and $z^{4}$, respectively, $\gamma_{\Sigma}$ coincides with the coefficient of $x^{2}$ divided by $z$, i.e., $\gamma_{\Sigma}=$ $a_{2,1}$. Since $\delta_{\Sigma}=4 a_{2,1}^{3} t$, it follows that

$$
\beta_{\Sigma}= \begin{cases}4 t, & \text { if } m \text { is odd } \\ -4 a_{2,1} t, & \text { if } m \text { is even }\end{cases}
$$

We summarize our first five examples in table 11.

We now give 15 additional examples, whose special fibers are of types $I V, I_{0}^{*}, I V^{*}, I I I^{*}$ or $I I^{*}$. The advantage of these types is that the equation of the special fiber is precisely the equation of an ADE singularity, and the example is part of the universal deformation of that singularity. (We choose the subspace of the universal deformation where the singularity corresponding to the general fiber is retained.) In all but one case, the rank of the corresponding Dynkin diagram jumps by 1 between general and special fiber, and the deformations we give are universal. We display this second group of examples in table 12 .

Note that the final column of the table indicates, which substitution $t=s^{k}$ must be made in order to get a map to the versal simultaneous resolution space for the family. For Examples 7-20, these are computed quite easily by using the fact that we have a universal deformation of a $D_{4}$ or an $E_{n}$ singularity. Those singularities have a weighted homogeneous equation, and have the property that the homogeneity can be extended to the universal deformation by giving the deformation parameter $t$ a weight (which is determined from the other data). These degrees of homogeneity are displayed in table 13. In each case $t$ has degree 1 or 2 , and that restricts the allowed basechanges. 
Table 12: Second group of examples. In \#7, $a$ and $b$ are constants.

\begin{tabular}{ccclc}
\hline & Gen. & Spc. & Equation & $t$ \\
\hline 6 & $I I I$ & $I V$ & $y^{2}=x^{3}+2 t z x+z^{2}$ & $s^{1 / 2}$ \\
7 & $I V$ & $I_{0}^{*}$ & $y^{2}=x^{3}+a z^{2} x+t z^{2}+b z^{3}$ & $s^{2}$ \\
8 & $I_{6}$ & $I V^{*}$ & $y^{2}=x^{3}+\left(t x+z^{2}\right)^{2}$ & $s^{2}$ \\
9 & $I_{7}$ & $I I I^{*}$ & $y^{2} x^{3}+\left(-4 t z+16 t^{3}\right) x^{2}$ & $s^{2}$ \\
& & & $+\left(z^{3}-8 t^{2} z^{2}\right) x+t z^{4}$ & $s^{2}$ \\
10 & $I_{8}$ & $I I^{*}$ & $y^{2}=x^{3}+\left(3 t^{2} z+t^{5}\right) x^{2}$ & \\
& & & $-\left(4 t z^{3}+2 t^{4} z^{2}\right) x+z^{5}+t^{3} z^{4}$ & $s$ \\
11 & $I_{1}^{*}$ & $I V^{*}$ & $y^{2}=x^{3}+t z x^{2}+z^{4}$ & $s^{2}$ \\
12 & $I_{2}^{*}$ & $I I I^{*}$ & $y^{2}=x^{3}+t z x^{2}+z^{3} x$ & $s^{2}$ \\
13 & $I_{3}^{*}$ & $I I^{*}$ & $y^{2}=x^{3}+z\left(t x+z^{2}\right)^{2}$ & $s$ \\
14 & $I V^{*}$ & $I I I^{*}$ & $y^{2}=x^{3}+z^{3} x+t z^{4}$ & $s^{2}$ \\
15 & $I I I^{*}$ & $I I^{*}$ & $y^{2}=x^{3}+t z^{3} x+z^{5}$ & $s^{2}$ \\
16 & $I_{2}+I_{5}$ & $I V^{*}$ & $y^{2}=x^{3}+\left(4 t z+t^{4}\right) x^{2}+2 t^{2} z^{2} x+z^{4}$ & $s^{2}$ \\
17 & $I_{2}+I_{1}^{*}$ & $I I I^{*}$ & $y^{2}=x^{3}+t z x^{2}+z^{3} x+t z^{4}$ & \\
18 & $I_{2}+I V^{*}$ & $I I^{*}$ & $y^{2}=x^{3}-3 t z^{3} x+z^{5}+t^{3} z^{4}$ & $s$ \\
19 & $I_{3}+I_{5}$ & $I I I^{*}$ & $y^{2}=x^{3}+\left(400 t^{3}-15 t z\right) x^{2}$ & \\
& & & & $+\left(480 t^{4} z-45 t^{2} z^{2}+z^{3}\right) x+144 t^{5} z^{2}-5 t^{3} z^{3}$ \\
20 & $I_{2}+I_{3}+I_{3}$ & $I V^{*}$ & $y^{2}=x^{3}-3 t^{4} x^{2}-6 t^{2} z^{2} x+16 t^{6} z^{2}+z^{4}$ & \\
\hline & & & &
\end{tabular}

Example 8.6. is slightly different: the special fiber

$$
y^{2}=x^{3}+z^{2}
$$

has a singularity of type $A_{2}$ and is weighted homogeneous, but the versal deformation

$$
y^{2}=x^{3}-s x^{2}+z^{2}
$$

does not coincide with our deformation. In fact, completing the square in our example, we get

$$
y^{2}=x^{3}-t^{2} x^{2}+(z+t x)^{2},
$$

which shows that $s=t^{2}$.

Most of these examples have appeared before in the literature $[8,9]$, but Examples 10, 13, and 16-20 are new. For each of these examples, we need to compute the Weierstrass coefficients $f$ and $g$ and the discriminant 
Table 13: Degrees of homogeneity degrees for Examples 6-20.

\begin{tabular}{lrrllc}
\hline Example & $x$ & $y$ & $z$ & $t$ & Eqn. \\
\hline 6 & 2 & 3 & 3 & 1 & 6 \\
7 & 2 & 3 & 2 & 2 & 6 \\
8 & 4 & 6 & 3 & 2 & 12 \\
9 & 6 & 9 & 4 & 2 & 18 \\
10 & 10 & 15 & 6 & 2 & 30 \\
11 & 4 & 6 & 3 & 1 & 12 \\
12 & 6 & 9 & 4 & 2 & 18 \\
13 & 10 & 15 & 6 & 2 & 30 \\
14 & 6 & 9 & 4 & 2 & 18 \\
15 & 10 & 15 & 6 & 2 & 30 \\
16 & 4 & 6 & 3 & 1 & 12 \\
17 & 6 & 9 & 4 & 2 & 18 \\
18 & 10 & 15 & 6 & 2 & 30 \\
19 & 6 & 9 & 4 & 2 & 18 \\
20 & 4 & 6 & 3 & 1 & 12 \\
\hline
\end{tabular}

$\Delta=4 f^{3}+27 g^{2}$, which we factor as much as possible. This factorization allows us to identify the components $\Sigma_{j}$ of the discriminant locus, which pass through the point $\{z=t=0\}$, and for each of these, we compute the quantities $\beta_{\Sigma_{j}}$ and $\gamma_{\Sigma_{j}}$ when defined (following table 7 ). All of these calculations are summarized in table 14. We describe these calculations in more detail in a few of the more challenging cases below, focusing on the examples, which are new.

We begin with Example 10, which has defining polynomial

$$
y^{2}=x^{3}+\left(3 t^{2} z+t^{5}\right) x^{2}-\left(4 t z^{3}+2 t^{4} z^{2}\right) x+z^{5}+t^{3} z^{4} .
$$

We complete the cube using $x=\widetilde{x}-t^{2} z-\frac{1}{3} t^{5}$, to obtain

$$
\begin{aligned}
y^{2}= & \widetilde{x}^{3}+\left(-4 t z^{3}-5 t^{4} z^{2}-2 t^{7} z-\frac{1}{3} t^{10}\right) \widetilde{x} \\
& +\left(z^{5}+5 t^{3} z^{4}+\frac{16}{3} t^{6} z^{3}+\frac{8}{3} t^{9} z^{2}+\frac{2}{3} t^{12} z+\frac{2}{27} t^{15}\right),
\end{aligned}
$$


Table 14: Calculations for the second group of examples. We use $\square$ to indicate an undefined quantity. In \#7, $a$ and $b$ are constants occurring in the equation, and $d=4 a^{3}+27 b^{2}$. In \#16-\#20, the factors in the discriminant and the $\left(\beta_{\Sigma}, \gamma_{\Sigma}\right)$ pairs are listed in the same order as the components of the general fiber in the left column.

\begin{tabular}{|c|c|c|c|c|c|}
\hline & Gen. Fib. & Discriminant & Type of $\Sigma$ & $\beta_{\Sigma}$ & $\gamma_{\Sigma}$ \\
\hline 6 & $I I I$ & $z^{3}\left(27 z+32 t^{3}\right)$ & $I I I$ & $\square$ & $2 t$ \\
\hline 7 & $I V$ & $z^{4}\left(d z^{2}+54 b t z+27 t^{2}\right)$ & $I V$ & $t$ & $\square$ \\
\hline 8 & $I_{6}$ & $z^{6}\left(27 z^{2}-4 t^{3}\right)$ & $I_{6}$ & $t^{2}$ & $-4 t^{-1}$ \\
\hline 9 & $I_{7}$ & $z^{7}\left(4 z^{2}-13 t^{2} z+32 t^{4}\right)$ & $I_{7}$ & $16 t^{3}$ & $\frac{1}{8} t^{-2}$ \\
\hline 10 & $I_{8}$ & $z^{8}\left(27 z^{2}+14 t^{3} z+3 t^{6}\right)$ & $I_{8}$ & $t^{5}$ & $3 t^{-4}$ \\
\hline 11 & $I_{1}^{*}$ & $z^{7}\left(27 z+4 t^{3}\right)$ & $I_{1}^{*}$ & 4 & $t$ \\
\hline 12 & $I_{2}^{*}$ & $z^{8}\left(4 z-t^{2}\right)$ & $I_{2}^{*}$ & 1 & $t$ \\
\hline 13 & $I_{3}^{*}$ & $z^{9}\left(27 z-4 t^{3}\right)$ & $I_{3}^{*}$ & $-4 t^{-3}$ & $t^{2}$ \\
\hline 14 & $I V^{*}$ & $z^{8}\left(4 z+27 t^{2}\right)$ & $I V^{*}$ & $t$ & $\square$ \\
\hline 15 & $I I I^{*}$ & $z^{9}\left(27 z+4 t^{3}\right)$ & $I I I^{*}$ & $\square$ & $t$ \\
\hline \multirow[t]{2}{*}{16} & $I_{2}+I_{5}$ & $\left(z+2 t^{3}\right)^{2} z^{5}\left(27 z+4 t^{3}\right)$ & $I_{2}$ & $\square$ & $64 t^{10}$ \\
\hline & & & $I_{5}$ & $t^{4}$ & $16 t$ \\
\hline \multirow[t]{2}{*}{17} & $I_{2}+I_{1}^{*}$ & $4\left(z+t^{2}\right)^{2} z^{7}$ & $I_{2}$ & $\square$ & $t^{8}$ \\
\hline & & & $I_{1}^{*}$ & $4 t$ & $t$ \\
\hline \multirow[t]{2}{*}{18} & $I_{2}+I V^{*}$ & $27\left(z-t^{3}\right)^{2} z^{8}$ & $I_{2}$ & $\square$ & $3 t^{14}$ \\
\hline & & & $I V^{*}$ & $t^{3}$ & $\square$ \\
\hline \multirow[t]{2}{*}{19} & $I_{3}+I_{5}$ & $z^{3}\left(z-32 t^{2}\right)^{5}\left(4 z-125 t^{2}\right)$ & $I_{3}$ & $400 t^{3}$ & $\frac{131072}{5} t$ \\
\hline & & & $I_{5}$ & $16 t^{3}$ & $-384 t^{2}$ \\
\hline \multirow[t]{3}{*}{20} & $I_{2}+I_{3}+I_{3}$ & $27 z^{2}\left(z-2 t^{3}\right)^{3}\left(z+2 t^{3}\right)^{3}$ & $I_{2}$ & $\square$ & $-192 t^{10}$ \\
\hline & & & $I_{3}$ & $9 t^{4}$ & $\frac{256}{3} t^{7}$ \\
\hline & & & $I_{3}$ & $9 t^{4}$ & $-\frac{256}{3} t^{7}$ \\
\hline
\end{tabular}

i.e., the Weierstrass coefficients are

$$
\begin{aligned}
& f=-4 t z^{3}-5 t^{4} z^{2}-2 t^{7} z-\frac{1}{3} t^{10} \\
& g=z^{5}+5 t^{3} z^{4}+\frac{16}{3} t^{6} z^{3}+\frac{8}{3} t^{9} z^{2}+\frac{2}{3} t^{12} z+\frac{2}{27} t^{15}
\end{aligned}
$$


Then the discriminant is:

$$
\begin{aligned}
& 4\left(-4 t z^{3}-5 t^{4} z^{2}-2 t^{7} z-\frac{1}{3} t^{10}\right)^{3} \\
& \quad+27\left(z^{5}+5 t^{3} z^{4}+\frac{16}{3} t^{6} z^{3}+\frac{8}{3} t^{9} z^{2}+\frac{2}{3} t^{12} z+\frac{2}{27} t^{15}\right)^{2} \\
& \quad=z^{8}\left(27 z^{2}+14 t^{3} z+3 t^{6}\right) .
\end{aligned}
$$

The relevant discriminant-component is $\{z=0\}$, along which we have a fiber of type $I_{8}$.

To calculate the other invariants for this example, we begin with

$$
\begin{aligned}
\left.f\right|_{\{z=0\}} & =-\frac{1}{3} t^{10}, \\
\left.g\right|_{\{z=0\}} & =\frac{2}{27} t^{15}, \\
\delta_{\{z=0\}} & =3 t^{6} .
\end{aligned}
$$

Thus $\beta_{\{z=0\}}=\left.(-9 g / 2 f)\right|_{\{z=0\}}=t^{5}$, and $\gamma_{\{z=0\}}=\delta_{\{z=0\}} / \beta_{\{z=0\}}^{2}=3 t^{-4}$.

Example 13 is quite straightforward. We start with the polynomial

$$
y^{2}=x^{3}+z\left(t x+z^{2}\right)^{2}=x^{3}+t^{2} z x^{2}+2 t z^{3} x+z^{5}
$$

and complete the cube using $x=\widetilde{x}-\frac{1}{3} t^{2} z$ to obtain the Weierstrass coefficients and discriminant

$$
\begin{aligned}
f & =z^{2}\left(2 t z-\frac{1}{3} t^{4}\right) \\
g & =z^{3}\left(z^{2}-\frac{2}{3} t^{3} z+\frac{2}{27} t^{6}\right), \\
\Delta & =z^{9}\left(27 z-4 t^{3}\right) .
\end{aligned}
$$

The relevant discriminant-component is $\{z=0\}$, along which we have a fiber of type $I_{3}^{*}$ (since $f$ and $g$ vanish to orders 2 and 3, respectively).

To calculate the other invariants,

$$
\begin{aligned}
\left.\left(f / z^{2}\right)\right|_{\{z=0\}} & =-\frac{1}{3} t^{4} \\
\left.\left(g / z^{3}\right)\right|_{\{z=0\}} & =\frac{2}{27} t^{6} \\
\delta_{\{z=0\}} & =-4 t^{3} .
\end{aligned}
$$

Thus $\gamma_{\{z=0\}}=\left.(-9 g / 2 z f)\right|_{\{z=0\}}=t^{2}$, and $\beta_{\{z=0\}}=\delta_{\{z=0\}} / \gamma_{\{z=0\}}^{3}=-4 t^{-3}$. 
Example 16 is the first case in which we get more than one relevant discriminant-component. We begin with the polynomial

$$
y^{2}=x^{3}+\left(4 t z+t^{4}\right) x^{2}+2 t^{2} z^{2} x+z^{4}
$$

We then complete the cube with the substitution $x=\tilde{x}-\frac{4}{3} t z-\frac{1}{3} t^{4}$ to obtain Weierstrass coefficients and discriminant:

$$
\begin{aligned}
f & =-\frac{1}{3}\left(10 t^{2} z^{2}+8 t^{5} z+t^{8}\right), \\
g & =\frac{1}{27}\left(27 z^{4}+56 t^{3} z^{3}+78 t^{6} z^{2}+24 t^{9} z+2 t^{12}\right), \\
\Delta & =\left(z+2 t^{3}\right)^{2} z^{5}\left(27 z+4 t^{3}\right) .
\end{aligned}
$$

Since none of the factors of the discriminant divide $f$ or $g$, we see that the Kodaira fiber types are indeed $I_{2}, I_{5}$ and $I_{1}$ along the three components of the discriminant locus. (Since the third of these does not contribute to the gauge algebra, we need not consider it in our computations.)

To compute the invariants along $\left\{z=-2 t^{3}\right\}$, we begin with

$$
\begin{aligned}
\left.f\right|_{\left\{z=-2 t^{3}\right\}} & =-\frac{1}{3}(40-16+1) t^{8}=-\frac{25}{3} t^{8}, \\
\left.g\right|_{\left\{z=-2 t^{3}\right\}} & =\frac{1}{27}(432-448+312-48+2) t^{12}=\frac{250}{27} t^{12}, \\
\delta_{\left\{z=-2 t^{3}\right\}} & =\left(-2 t^{3}\right)^{5}\left(-54 t^{3}+4 t^{3}\right)=1600 t^{18} .
\end{aligned}
$$

Then

$$
\begin{aligned}
\left.u\right|_{\left\{z=-2 t^{3}\right\}} & =\left.(-9 g / 2 f)\right|_{\left\{z=-2 t^{3}\right\}}=5 t^{4} \\
\gamma_{\left\{z=-2 t^{3}\right\}} & =\delta_{\left\{z=-2 t^{3}\right\}} /\left(\left.u\right|_{\left\{z=-2 t^{3}\right\}}\right)^{2}=64 t^{10} .
\end{aligned}
$$

On the other hand, to compute the invariants along $\{z=0\}$, we begin with

$$
\begin{aligned}
\left.f\right|_{\{z=0\}} & =-\frac{1}{3} t^{8}, \\
\left.g\right|_{\{z=0\}} & =\frac{2}{27} t^{12}, \\
\delta_{\{z=0\}} & =\left(2 t^{3}\right)^{2}\left(4 t^{3}\right)=16 t^{9} .
\end{aligned}
$$

Thus, $\beta_{\{z=0\}}=\left.(-9 g / 2 f)\right|_{\{z=0\}}=t^{4}$ and $\gamma_{\{z=0\}}=\delta_{\{z=0\}} / \beta_{\{z=0\}}^{2}=16 t$. 
For Example 17, we complete the cube on

$$
y^{2}=x^{3}+t z x^{2}+z^{3} x+t z^{4}
$$

and find that

$$
\begin{aligned}
& f=z^{2}\left(z-\frac{1}{3} t^{2}\right) \\
& g=z^{3}\left(\frac{2}{3} t z+\frac{2}{27} t^{3}\right) \\
& \Delta=4\left(z+t^{2}\right)^{2} z^{7}
\end{aligned}
$$

from which we can easily compute the invariants along the two components of the discriminant. For $\left\{z=-t^{2}\right\}$ we have type $I_{2}$ and

$$
\left.u\right|_{\left\{z=-t^{2}\right\}}=\frac{-\left.9 g\right|_{\left\{z=-t^{2}\right\}}}{\left.2 f\right|_{\left\{z=-t^{2}\right\}}}=\frac{-9 \cdot(16 / 27) t^{9}}{-2 \cdot(4 / 3) t^{6}}=2 t^{3},
$$

while $\delta_{\left\{z=-t^{2}\right\}}=4 t^{14}$ so that $\gamma_{\left\{z=-t^{2}\right\}}=4 t^{14} /\left(2 t^{3}\right)^{2}=t^{8}$.

On the other hand, for $\{z=0\}$ we have type $I_{1}^{*}$ and

$$
\gamma_{z=0}=\left.(u / z)\right|_{\{z=0\}}=\frac{-9 \cdot(2 / 27) t^{3}}{2 \cdot(-1 / 3) t^{2}}=t .
$$

Since $\delta_{\{z=0\}}=4 t^{4}$, we have $\beta_{\{z=0\}}=4 t^{4} / t^{3}=4 t$.

Examples 18 and 19 are quite similar to these.

Example 20 is the most complicated one. We start with

$$
y^{2}=x^{3}-3 t^{4} x^{2}-6 t^{2} z^{2} x+16 t^{6} z^{2}+z^{4}
$$

and complete the cube with $x=\tilde{x}+t^{4}$ to obtain

$$
y^{2}=\tilde{x}^{3}+\left(-6 t^{2} z^{2}-3 t^{8}\right) \tilde{x}+\left(z^{4}+10 t^{6} z^{2}-2 t^{12}\right) .
$$

Thus, the discriminant is

$$
4\left(-6 t^{2} z^{2}-3 t^{8}\right)^{3}+27\left(z^{4}+10 t^{6} z^{2}-2 t^{12}\right)^{2}=27 z^{2}\left(z-2 t^{3}\right)^{3}\left(z+2 t^{3}\right)^{3} .
$$

There are three relevant components of the discriminant locus, with fibers of types $I_{2}, I_{3}$, and $I_{3}$. 
We have

$$
\begin{aligned}
\left.f\right|_{\{z=0\}} & =-3 t^{8}, \\
\left.g\right|_{\{z=0\}} & =-2 t^{12}, \\
\delta_{\{z=0\}} & =-27 \cdot 64 t^{18},
\end{aligned}
$$

and so $\left.u\right|_{\{z=0\}}=\left.(-9 g / 2 f)\right|_{\{z=0\}}=-3 t^{4}$, which implies that $\gamma_{\{z=0\}}=\delta_{\{z=0\}} /$ $\left(\left.u\right|_{\{z=0\}}\right)^{2}=-192 t^{10}$.

On the other hand,

$$
\begin{aligned}
\left.f\right|_{\left\{z= \pm 2 t^{3}\right\}} & =(-24-3) t^{8}=-27 t^{8} \\
\left.g\right|_{\left\{z= \pm 2 t^{3}\right\}} & =(16+40-2) t^{12}=54 t^{12} \\
\delta_{\left\{z= \pm 2 t^{3}\right\}} & =27\left( \pm 2 t^{3}\right)^{2}\left( \pm 4 t^{3}\right)^{3}= \pm 27 \cdot 256 t^{15}
\end{aligned}
$$

so $\quad \beta_{\left\{z= \pm 2 t^{3}\right\}}=\left.(-9 g / 2 f)\right|_{\left\{z= \pm 2 t^{3}\right\}}=9 t^{4} \quad$ and $\quad \gamma_{\left\{z= \pm 2 t^{3}\right\}}=\delta_{\left\{z= \pm 2 t^{3}\right\}} /$ $\beta_{\left\{z= \pm 2 t^{3}\right\}}^{2}= \pm \frac{256}{3} t^{7}$.

Each of our new examples was constructed using the data from [57], specialized to the particular geometric situation we were constructing, and the starting point for each of these derivations was a subdiagram of a Dynkin diagram. For this reason, we expect that the matter representations associated with these examples should be obtained from the algebra inclusion corresponding to those Dynkin sub-diagrams. In the one ambiguous case (Example 10), this leads to a precise prediction for the matter representation: it should be fund $\oplus \Lambda^{2} \oplus \Lambda^{3}$.

To analyze the matter representation for each of these examples, we take the inclusion of (simply laced) Dynkin diagrams $R \subset R^{\prime}$, which corresponds to the degeneration in the example, and decompose the adjoint representation of the Lie algebra $\mathfrak{g}\left(R^{\prime}\right)$ under the action of the Lie algebra $\mathfrak{g}(R)$. The non-trivial constituents other than the adjoint representation of $\mathfrak{g}(R)$, when restricted to the Lie algebra $\mathfrak{g}$, comprise the corresponding matter representation. This is determined by means of "branching rules" for the adjoint representation, which are easily obtained from a reference such as [45] or [46]. We collect the information we need in table 15 (most of which was already presented in [10]). The table lists a representation $\rho_{R, R^{\prime}}$ with the property that $\operatorname{adj}_{\mathfrak{g}\left(R^{\prime}\right)}$ decomposes as a representation over $\mathfrak{g}(R)$ into

$$
\operatorname{adj}_{\mathfrak{g}\left(R^{\prime}\right)}=\operatorname{adj}_{\mathfrak{g}(R)} \oplus \rho_{R, R^{\prime}} \oplus \overline{\rho_{R, R^{\prime}}} \oplus \mathbf{1}^{\oplus\left(\operatorname{rank}\left(R^{\prime}\right)-\operatorname{rank}(R)\right)} .
$$

(Note that if $\mathfrak{g}$ is not simply laced then $\mathfrak{g}(R)$ is the covering algebra and the actual matter representation is $\left.\rho_{R, R^{\prime}}\right|_{\mathfrak{g}}$.) In the table, we have included 
Table 15: Branching rules for the examples.

\begin{tabular}{|c|c|c|c|}
\hline & $R$ & $R^{\prime}$ & $\rho_{R, R^{\prime}}$ \\
\hline 1 & $A_{m-1}$ & $A_{m}$ & fund \\
\hline 2 & $A_{2 n-1}$ & $D_{2 n}$ & $\Lambda^{2}$ \\
\hline 3 & $A_{2 n}$ & $D_{2 n+2}$ & fund $^{\oplus 2} \oplus \Lambda^{2}$ \\
\hline 4 & $A_{2 n}$ & $D_{2 n+1}$ & $\Lambda^{2}$ \\
\hline 5 & $D_{m}$ & $D_{m+1}$ & vect \\
\hline 6 & $A_{1}$ & $A_{2}$ & fund \\
\hline 7 & $A_{2}$ & $D_{4}$ & fund $^{\oplus 3}$ \\
\hline 8 & $A_{5}$ & $E_{6}$ & $\mathbf{1} \oplus \Lambda^{3}$ \\
\hline 9 & $A_{6}$ & $E_{7}$ & fund $\oplus \Lambda^{3}$ \\
\hline 10 & $A_{7}$ & $E_{8}$ & fund $\oplus \Lambda^{2} \oplus \Lambda^{3}$ \\
\hline $10^{\prime}$ & $A_{7}$ & $E_{8}$ & $\mathbf{1} \oplus\left(\Lambda^{2}\right)^{\oplus 2} \oplus \frac{1}{2} \Lambda^{4}$ \\
\hline 11 & $D_{5}$ & $E_{6}$ & $\operatorname{spin}_{+}$ \\
\hline 12 & $D_{6}$ & $E_{7}$ & $1 \oplus \operatorname{spin}_{+}$ \\
\hline 13 & $D_{7}$ & $E_{8}$ & vect $\oplus \operatorname{spin}_{+}$ \\
\hline 14 & $E_{6}$ & $E_{7}$ & 27 \\
\hline 15 & $E_{7}$ & $E_{8}$ & $1 \oplus 56$ \\
\hline 16 & $A_{1}+A_{4}$ & $E_{6}$ & $(\mathbf{1} \otimes$ fund $) \oplus\left(\right.$ fund $\left.\otimes \Lambda^{2}\right)$ \\
\hline 17 & $A_{1}+D_{5}$ & $E_{7}$ & $(\mathbf{1} \otimes$ vect $) \oplus\left(\right.$ fund $\left.\otimes \operatorname{spin}_{+}\right)$ \\
\hline 18 & $A_{1}+E_{6}$ & $E_{8}$ & $(\mathbf{1} \otimes \mathbf{2 7}) \oplus($ fund $\otimes \mathbf{1}) \oplus($ fund $\otimes \mathbf{2 7})$ \\
\hline 19 & $A_{2}+A_{4}$ & $E_{7}$ & $(\mathbf{1} \otimes$ fund $) \oplus($ fund $\otimes$ fund $) \oplus\left(\right.$ fund $\left.\otimes \Lambda^{2}\right)$ \\
\hline 20 & $A_{1}+A_{2}+A_{2}$ & $E_{6}$ & $\begin{array}{c}(\mathbf{1} \otimes \text { fund } \otimes \text { fund }) \oplus(\text { fund } \otimes \mathbf{1} \otimes \mathbf{1}) \\
\oplus(\text { fund } \otimes \text { fund } \otimes \text { fund })\end{array}$ \\
\hline
\end{tabular}

two entries for $A_{7} \subset E_{8}$, since there are known to be two different algebra embeddings, and their representation theory differs.

In addition to the ambiguity of embedding $A_{7}$ into $E_{8}$, there is a second ambiguity: there are two embeddings of $D_{6}$ into $E_{7}$ (or of $I_{2}^{*}$ into $I I I$ ) one of which yields spin $_{+}$in the decomposition and the other of which yields spin_. There is an isomorphism which maps one of these representations to the other, and it can be realized in the geometry as well. That is, depending on how we label the roots and weights of $D_{6}$ as geometric objects, either representation can occur.

\section{Anomaly cancellation}

We now verify that each of our examples satisfies anomaly cancellation. That is, for each type of singular point, we compute the contributions to the 
Tate cycles and compare them to the contribution to the matter cycle; we also compute intersection numbers for all pairs of discriminant components meeting at the point.

- Example 1 has fibers of type $I_{m}$ along $\Sigma$ with no monodromy at the special point $t=0$, so it can represent either $\mathfrak{s u}(m)$ or $\mathfrak{s p}([m / 2]) \cdot \gamma_{\Sigma}$ has a zero of order 1 and $\beta_{\Sigma}$ is non-vanishing, so by consulting table 9 we see that the Tate representation is the fundamental representation (for either gauge algebra, and any parity of $m$ ). On the other hand, since no basechange is required for simultaneous resolution (i.e., $k=1$ ), table 15 implies that the matter representation is also the fundamental representation.

- Example 2 has fibers of type $I_{2 n}$ along $\Sigma$ with monodromy at the special point. Thus, this example is naturally associated with the gauge algebra $\mathfrak{s p}(n)$, and we have $\gamma_{\Sigma}$ non-vanishing but $\beta_{\Sigma}$ has a zero of order 1. From table 9 we see that the Tate representation is $\frac{1}{2} \Lambda_{\text {irr }}^{2}$. On the other hand, $k=2$ so table 15 shows that we get the charged part of $\left.\frac{1}{2} \Lambda^{2}\right|_{\mathfrak{s p}(n)}=\frac{1}{2} \mathbf{1} \oplus \frac{1}{2} \Lambda_{\text {irr }}^{2}$ for the matter representation. Discarding the uncharged part of the representation, we find agreement.

Note that because of the monodromy, we expect this representation to contain $\frac{1}{2} \rho_{0}$ as a summand. In fact, the representation coincides with $\frac{1}{2} \rho_{0}$ in this case.

- If we make a basechange $t=s^{2}$ in Example 2, we get an example without monodromy, which is suitable for the gauge algebra $\mathfrak{s u}(2 n)$. This time $\beta_{\Sigma}$ vanishes to order 2 while $\gamma_{\Sigma}$ is non-vanishing, so the Tate representation is $\Lambda^{2}$. In this case, $k=1$ so by table 15 the matter representation is also $\Lambda^{2}$.

- Example 3 has fibers of type $I_{2 n+1}$ along $\Sigma$ with monodromy at the special point, so that this example is associated with the gauge algebra $\mathfrak{s p}(n)$. Both $\beta_{\Sigma}$ and $\gamma_{\Sigma}$ have zeros of order 1 . Thus, in table 9 we must add together two representations to find the Tate representation: $\frac{1}{2} \Lambda_{\text {irr }}^{2}+\frac{3}{2}$ fund. On the other hand, since $k=2$, restricting the appropriate entry from table 15 to $\mathfrak{s p}(n)$ we find matter representation

$$
\left.\left(\text { fund }_{\mathfrak{s u}(2 n+1)}+\frac{1}{2} \Lambda_{\mathfrak{s u}(2 n+1)}^{2}\right)\right|_{\mathfrak{s p}(n)}=\mathbf{1}+\text { fund }+\frac{1}{2} \cdot \mathbf{1}+\frac{1}{2} \text { fund }+\frac{1}{2} \Lambda_{\mathrm{irr}}^{2} .
$$

Thus, the charged parts agree. 
In this case, we can write the representation in the form $\frac{1}{2} \rho_{0}+$ $\frac{1}{2}$ fund, and since the fundamental representation is quarternionic, $\frac{1}{2}$ fund indeed defines a pre-quaternionic representation as expected.

- Example 4 has fibers of type $I_{2 n+1}$ along $\Sigma$ with no local monodromy at the special point; thus, this example can be associated with either the gauge algebra $\mathfrak{g}(\Sigma)=\mathfrak{s u}(2 n+1)$ or the gauge algebra $\mathfrak{g}(\Sigma)=\mathfrak{s p}(n)$. $\beta_{\Sigma}$ vanishes to order 2 and $\gamma_{\Sigma}$ is non-vanishing, so table 9 tells us that the charged part of the Tate representation is $\left.\Lambda^{2}\right|_{\mathfrak{g}(\Sigma)}$. On the other hand, since $k=1$, table 15 shows that the charged part of the matter representation is also $\left.\Lambda^{2}\right|_{\mathfrak{g}(\Sigma)}$.

- Example 5 has fibers of type $I_{m-4}^{*}$ along $\Sigma$ with local monodromy at the special point, so it must represent the gauge algebra $\mathfrak{s o}(2 m-1) \cdot \beta_{\Sigma}$ has a zero of order 1 and $\gamma_{\Sigma}$ is non-vanishing, so by consulting table 9 we see that the Tate representation is $\frac{1}{2}$ vect. On the other hand, $k=2$ so by table 15 we find that the matter representation is also $\frac{1}{2}$ vect. As in Example 2, this representation also coincides with $\frac{1}{2} \rho_{0}$.

- If we make a basechange $t=s^{2}$ in Example 5, we get an example without monodromy, which is suitable for the gauge algebra $\mathfrak{s o}(2 m)$. This time $\beta_{\Sigma}$ vanishes to order 2 while $\gamma_{\Sigma}$ is non-vanishing, so the Tate representation is the vector representation. In this case $k=1$ so by table 15 the matter representation is also the vector representation.

- Example 6 has fibers of type $I I I$ along $\Sigma$ with no local monodromy, and the gauge algebra is $\mathfrak{s u}(2) . \gamma_{\Sigma}$ has a zero of order 1 , so by table 9 , the Tate representation is 2 -fund. On the other hand, since $k=\frac{1}{2}$, from table 15 we see that the matter representation is also 2 . fund.

- Example 7 has fibers of type $I V$ along $\Sigma$ with local monodromy, and so is appropriate for the gauge algebra $\mathfrak{s p}(1) . \beta_{\Sigma}$ has a zero of order 1 , so by table 9 , the Tate representation is $\frac{3}{2}$ - fund. On the other hand, since $k=2$, from table 15 we see that the matter representation is also $\frac{3}{2}$. fund. Once again, we have monodromy and this coincides with $\frac{1}{2} \rho_{0}$.

- If we make a basechange $t=s^{2}$ in Example 7, we get an example without monodromy, which is suitable for the gauge algebra $\mathfrak{s u}(3)$. This time $\beta_{\Sigma}$ has a zero of order 2 , so the Tate representation is $3 \cdot$ fund. In this case $k=1$ so by table 15 the matter representation is also 3 - fund.

- Example 8 has fibers of type $I_{6}$ along $\Sigma$ without local monodromy, and so is appropriate for either the gauge algebra $\mathfrak{g}(\Sigma)=\mathfrak{s u}(6)$ or the 
gauge algebra $\mathfrak{g}(\Sigma)=\mathfrak{s p}(3) . \beta_{\Sigma}$ has a zero of order 2 , and $\gamma_{\Sigma}$ has a pole of order 1 , so the Tate representation is determined as the difference of two entries in table 9: it is the charged part of

$$
\left.\Lambda^{2}\right|_{\mathfrak{g}(\Sigma)}-\text { fund }\left.\right|_{\mathfrak{g}(\Sigma)}
$$

On the other hand, since $k=2$, we see from table 15 that the matter representation is the charged part of $\left.\frac{1}{2} \Lambda^{3}\right|_{\mathfrak{g}(\Sigma)}$, that is,

$$
\rho_{\text {matter }}= \begin{cases}\frac{1}{2} \Lambda^{3}, & \text { if } \mathfrak{g}(\Sigma)=\mathfrak{s u}(6) \\ \frac{1}{2} \Lambda_{\text {irr }}^{3}+\frac{1}{2} \text { fund, }, & \text { if } \mathfrak{g}(\Sigma)=\mathfrak{s p}(3)\end{cases}
$$

Note that $\Lambda^{3}$ is a quaternionic representation of $\mathfrak{s u}(6)$, and that both $\Lambda_{\text {irr }}^{3}$ and fund are quaternionic representations of $\mathfrak{s p}(3)$, so this matter representation is pre-quaternionic.

To verify anomaly cancellation in this case, we need to use Casimir equivalence: by the first and fifth lines in table 3 , the Tate representation (9.1) is Casimir equivalent to the matter representation (9.2) for both gauge algebras.

- Example 9 has fibers of type $I_{7}$ along $\Sigma$ with local monodromy, and so is appropriate for the gauge algebra $\mathfrak{g}(\Sigma)=\mathfrak{s p}(3)$. $\beta_{\Sigma}$ has a zero of order 3 , and $\gamma_{\Sigma}$ has a pole of order 2 , so the Tate representation is determined as the difference of two entries in table 9: it is

$$
\frac{3}{2} \Lambda_{\text {irr }}^{2}+\frac{3}{2} \text { fund }-2 \text { fund }=\frac{3}{2} \Lambda_{\text {irr }}^{2}-\frac{1}{2} \text { fund } .
$$

On the other hand, since $k=2$, we see from table 15 that the matter representation is

(9.4) $\left.\left(\frac{1}{2}\right.$ fund $\left._{\mathfrak{s u}(7)}+\frac{1}{2} \Lambda_{\mathfrak{s u}(7)}^{3}\right)\right|_{\mathfrak{s p}(3)}=$ fund $+\frac{1}{2} \Lambda_{\text {irr }}^{2}+\frac{1}{2} \Lambda_{\text {irr }}^{3}=\frac{1}{2} \rho_{0}+\frac{1}{2} \Lambda_{\text {irr }}^{3}$.

Since $\Lambda_{\text {irr }}^{3}$ is quaternionic, $\frac{1}{2} \Lambda_{\text {irr }}^{3}$ does indeed define a pre-quaternionic representation as expected.

To verify anomaly cancellation, we again employ table 3 to conclude that $\frac{1}{2} \Lambda_{\text {irr }}^{3}$ is Casimir equivalent to $\Lambda_{\text {irr }}^{2}-\frac{3}{2}$ fund. Thus, the matter 
representation is Casimir equivalent to

$$
\text { fund }+\frac{1}{2} \Lambda_{\text {irr }}^{2}+\Lambda_{\text {irr }}^{2}-\frac{3}{2} \text { fund }=\frac{3}{2} \Lambda_{\text {irr }}^{2}-\frac{1}{2} \text { fund }
$$

i.e., to the Tate representation for $\Sigma$.

- If we make a basechange $t=s^{2}$ in Example 9, we get an example without monodromy, which is suitable for the gauge algebra $\mathfrak{s u}(7)$. This time $\beta_{\Sigma}$ has a zero of order 6 , and $\gamma_{\Sigma}$ has a pole of order 4 , so the Tate representation is determined as the difference $3 \cdot \Lambda^{2}-4 \cdot$ fund. In this case $k=1$ so by table 15 the matter representation is fund $+\Lambda^{3}$. Now the second line in table 3 shows that these are Casimir equivalent.

- Example 10 has fibers of type $I_{8}$ along $\Sigma$ with local monodromy, and so is appropriate for the gauge algebra $\mathfrak{g}(\Sigma)=\mathfrak{s p}(4) . \beta_{\Sigma}$ has a zero of order 5 , and $\gamma_{\Sigma}$ has a pole of order 4 , so the Tate representation is determined as the difference of two entries in table 9: it is the charged part of

$$
\frac{5}{2} \Lambda_{\text {irr }}^{2}-4 \text { fund }
$$

On the other hand, since $k=2$, we see from table 15 that the matter representation takes one of two forms: it is either (1) the charged part of

$$
\left.\frac{1}{2}\left(\text { fund }+\Lambda^{2}+\Lambda^{3}\right)\right|_{\mathfrak{s p}(4)}=\frac{1}{2} \cdot \mathbf{1}+\text { fund }+\frac{1}{2} \Lambda_{\text {irr }}^{2}+\frac{1}{2} \Lambda_{\text {irr }}^{3},
$$

which can be written in the form

$$
\frac{1}{2} \rho_{0}+\text { fund }+\frac{1}{2} \Lambda_{\text {irr }}^{3},
$$

or (2) the charged part of

$$
\left.\frac{1}{2}\left(\mathbf{1}+2 \cdot \Lambda^{2}+\frac{1}{2} \Lambda^{4}\right)\right|_{\mathfrak{s p}(4)}=\frac{7}{4} \cdot \mathbf{1}+\frac{5}{4} \Lambda_{\mathrm{irr}}^{2}+\frac{1}{4} \Lambda_{\mathrm{irr}}^{4}
$$

which can be written in the form

$$
\frac{1}{2} \rho_{0}+\frac{3}{4} \Lambda_{\mathrm{irr}}^{2}+\frac{1}{4} \Lambda_{\mathrm{irr}}^{4}
$$

The second case does not leave us with a pre-quaternionic representation, so does not appear to be valid choice for the matter representation 
(although it is Casimir equivalent to the Tate representation, as follows from the last line of table 3 ).

On the other hand, since $\Lambda_{\text {irr }}^{3}$ is quaternionic, fund $+\frac{1}{2} \Lambda_{\text {irr }}^{3}$ defines a pre-quaternionic representation. Moreover, anomaly cancellation works because $\frac{1}{2} \Lambda_{\text {irr }}^{3}$ is Casimir equivalent to $2 \cdot \Lambda_{\text {irr }}^{2}-5 \cdot$ fund.

- If we make a basechange $t=s^{2}$ in Example 10, we get an example without monodromy, which is suitable for the gauge algebra $\mathfrak{s u}(8)$. This time $\beta_{\Sigma}$ has a zero of order 10 , and $\gamma_{\Sigma}$ has a pole of order 8 , so the Tate representation is determined as the difference $5 \cdot \Lambda^{2}-8 \cdot$ fund. In this case $k=1$ so by table 15 the matter representation is the charged part of either fund $+\Lambda^{2}+\Lambda^{3}$ or $\mathbf{1}+2 \cdot \Lambda^{2}+\frac{1}{2} \Lambda^{4}$. Now the third line in table 3 shows that $\Lambda^{3}$ is Casimir equivalent to $4 \cdot \Lambda^{2}-9 \cdot$ fund, from which the anomaly cancellation follows in the first case; the fourth line shows that $\Lambda^{4}$ is Casimir equivalent to $6 \cdot \Lambda^{2}$. $-16 \cdot$ fund, from which the anomaly cancellation follows in the second case. Thus, either choice seems a priori possible, but as we stated near the end of Section 8, our method of construction of this example strongly suggests that the correct matter representation is fund $+\Lambda^{2}+\Lambda^{3}$.

- Example 11 has fibers of type $I_{1}^{*}$ along $\Sigma$ without local monodromy, and so is appropriate either for the gauge algebra $\mathfrak{s o}(9)$ or for the gauge algebra $\mathfrak{s o}(10) . \beta_{\Sigma}$ is non-vanishing, and $\gamma_{\Sigma}$ has a zero of order 1 , so by table 9 , the Tate representation is $\operatorname{spin}_{*}$. On the other hand, since $k=1$, from table 15 we see that the matter representation is also $\operatorname{spin}_{*}$. (More precisely the matter representation in the case of $\mathfrak{s o}(10)$ can be written as either $\operatorname{spin}_{+}$or $\operatorname{spin}_{-}$, which give the same quaternionic representation since they are complex conjugates of each other. The representation for $\mathfrak{s o}(9)$ is the restriction of either of these to $\mathfrak{s o}(9)$, which is spin in either case.)

- Example 12 has fibers of type $I_{2}^{*}$ along $\Sigma$ without local monodromy, and so is appropriate either for the gauge algebra $\mathfrak{s o}(11)$ or for the gauge algebra $\mathfrak{s o}(12) . \beta_{\Sigma}$ is non-vanishing, and $\gamma_{\Sigma}$ has a zero of order 1 , so by table 9 , the Tate representation is $\frac{1}{2} \operatorname{spin}_{*}$. On the other hand, since $k=2$, from table 15 we see that the charged part of the matter representation is also $\frac{1}{2} \operatorname{spin}_{*}$. Note that $\operatorname{spin}_{*}$ is quaternionic so that $\frac{1}{2} \operatorname{spin}_{*}$ is a pre-quaternionic representation.

- Example 13 has fibers of type $I_{3}^{*}$ along $\Sigma$ with local monodromy, and so is appropriate for the gauge algebra $\mathfrak{s o}(13) . \beta_{\Sigma}$ has a pole of order 3 , and $\gamma_{\Sigma}$ has a zero of order 2 , so the Tate representation is a difference 
of entries in table 9:

$$
-\frac{3}{2} \cdot \operatorname{vect}+\frac{1}{2} \cdot \operatorname{spin}_{*}+2 \cdot \operatorname{vect}=\frac{1}{2} \cdot \operatorname{spin}_{*}+\frac{1}{2} \cdot \text { vect } .
$$

On the other hand, since $k=2$, from table 15 we see that the matter representation is also

$$
\frac{1}{2} \cdot \operatorname{vect}+\frac{1}{2} \cdot \operatorname{spin}_{*}=\frac{1}{2} \rho_{0}+\frac{1}{2} \operatorname{spin}_{*} \cdot
$$

Since $\operatorname{spin}_{*}$ is quaternionic, $\frac{1}{2} \operatorname{spin}_{*}$ defines a pre-quaternionic representation as expected.

- If we make a basechange $t=s^{2}$ in Example 13, we get an example without monodromy, which is suitable for the gauge algebra $\mathfrak{s o}(14)$. This time $\beta_{\Sigma}$ has a pole of order 6 , and $\gamma_{\Sigma}$ has a zero of order 4 , so the Tate representation is a difference of entries in table 9:

$$
-3 \cdot \operatorname{vect}+\operatorname{spin}_{*}+4 \cdot \operatorname{vect}=\operatorname{vect}+\operatorname{spin}_{*} .
$$

In this case $k=1$ so by table 15 the matter representation is also vect $+\operatorname{spin}_{*}$.

- Example 14 has fibers of type $I V^{*}$ along $\Sigma$ with local monodromy, and so is appropriate for the gauge algebra $\mathfrak{f}_{4} \cdot \beta_{\Sigma}$ has a zero of order 1 , so by table 9 , the Tate representation is $\frac{1}{2} \cdot \mathbf{2 6}$. On the other hand, since $k=2$, from table 15 we see that the the matter representation is the charged part of

$$
\left.\frac{1}{2} \cdot \mathbf{2 7}\right|_{\mathfrak{f}_{4}}=\frac{1}{2} \cdot \mathbf{1}+\frac{1}{2} \cdot \mathbf{2 6}
$$

the same as the Tate representation. Again, as is typical with monodromy, this representation coincides with $\frac{1}{2} \rho_{0}$.

- If we make a basechange $t=s^{2}$ in Example 14, we get an example without monodromy, which is suitable for the gauge algebra $\mathfrak{e}_{6}$. This time $\beta_{\Sigma}$ has a zero of order 2 , so by table 9 , the Tate representation is 27. In this case $k=1$ so by table 15 the matter representation is also $\mathbf{2 7}$, the same as the Tate representation.

- Example 15 has fibers of type $I I I^{*}$ along $\Sigma$ without local monodromy, and is appropriate for the gauge algebra $\mathfrak{e}_{7} \cdot \beta_{\Sigma}$ is non-vanishing, and $\gamma_{\Sigma}$ has a zero of order 1 , so by table 9 , the Tate representation is $\frac{1}{2} \cdot \mathbf{5 6}$. 
On the other hand, since $k=2$, from table 15 we see that the matter representation is also $\frac{1}{2} \mathbf{5 6}$. Since $\mathbf{5 6}$ is a quaternionic representation, this is pre-quaternionic.

- Example 16 has fibers of type $I_{2}$ along $\Sigma$ and fibers of type $I_{5}$ along $\Sigma^{\prime}$, which meet at a common point. There is no local monodromy along $\Sigma$, so either gauge algebra $\mathfrak{g}(\Sigma) \oplus \mathfrak{g}\left(\Sigma^{\prime}\right)=\mathfrak{s u}(2) \oplus \mathfrak{s p}(2)$ or $\mathfrak{g}(\Sigma) \oplus \mathfrak{g}\left(\Sigma^{\prime}\right)=$ $\mathfrak{s u}(2) \oplus \mathfrak{s u}(5)$ is possible; we will make the calculation for the second one. We need to calculate the Tate representation for each component, as well as the representation-multiplicity for the pair: the data for this are contained in table 14 . Along $\Sigma, \gamma_{\Sigma}$ has a zero of order 10 , so the Tate representation for $\Sigma$ is 10 . fund (a representation of $\mathfrak{s u}(2)$ ). On the other hand, along $\Sigma^{\prime}, \beta_{\Sigma^{\prime}}$ has a zero of order 4 and $\gamma_{\Sigma^{\prime}}$ has a zero of order 1 , so adding the corresponding entries in table 9 we see that the Tate representation for $\Sigma^{\prime}$ is $2 \Lambda^{2}+$ fund (a representation of $\mathfrak{s u}(5)$ ).

The expression for the discriminant of this example from table 14 shows that the components $\Sigma$ and $\Sigma^{\prime}$ have intersection multiplicity 3 ; thus, we need

$$
\frac{\operatorname{Tr}_{\rho}\left(F_{\mathfrak{g}(\Sigma)}^{2}\right) \operatorname{Tr}_{\rho}\left(F_{\mathfrak{g}\left(\Sigma^{\prime}\right)}^{2}\right)}{\operatorname{tr}_{\mathfrak{g}(\Sigma)}\left(F^{2}\right) \operatorname{tr}_{\mathfrak{g}\left(\Sigma^{\prime}\right)}\left(F^{2}\right)}=3
$$

for the representation $\rho$ associated with our special point $z=t=0$.

Now we turn to representation theory to compute the actual matter representation. As indicated in table 15 , since $k=1$, the matter representation is

$$
\rho=(\mathbf{1} \otimes \text { fund }) \oplus\left(\text { fund } \otimes \Lambda^{2}\right) .
$$

There are three things to check in order to verify anomaly cancellation:

1. When the matter representation is restricted to $\mathfrak{g}(\Sigma)=\mathfrak{s u}(2)$, the charged part is ten copies of the fundamental, since $\Lambda_{\mathfrak{s u}(5)}^{2}$ has dimension 10. This agrees with the Tate representation for $\Sigma$.

2. When restricted to $\mathfrak{g}\left(\Sigma^{\prime}\right)=\mathfrak{s u}(5)$, the charged part is 2 copies of $\Lambda^{2}$ plus a fundamental, since the fundamental of $\mathfrak{s u}(2)$ has dimension 2. This agrees with the Tate representation for $\Sigma^{\prime}$.

3. Since

$$
\begin{aligned}
\operatorname{Tr}_{\text {fund }_{\mathfrak{s u}(2)}} F^{2}=\operatorname{tr}_{\mathfrak{s u}(2)} F^{2} \\
\operatorname{Tr}_{\Lambda_{\mathfrak{s u}(5)}^{2}} F^{2}=3 \operatorname{tr}_{\mathfrak{s u}(5)} F^{2},
\end{aligned}
$$


we have $\mu_{\rho}\left(\mathfrak{g}(\Sigma), \mathfrak{g}\left(\Sigma^{\prime}\right)\right)=1 \cdot 3=3$, so the representation-multiplicity coincides with the intersection multiplicity.

Thus, the anomalies cancel.

- Example 17 has fibers of type $I_{2}$ along $\Sigma$ and fibers of type $I_{1}^{*}$ along $\Sigma^{\prime}$, which meet at a common point. There is local monodromy along $\Sigma^{\prime}$, so the gauge algebra must be $\mathfrak{s u}(2) \oplus \mathfrak{s o}(9)$. According to table 14 , along $\Sigma, \gamma_{\Sigma}$ has a zero of order 8 , so the Tate representation for $\Sigma$ is 8 . fund. On the other hand, along $\Sigma^{\prime}, \beta_{\Sigma^{\prime}}$ and $\gamma_{\Sigma^{\prime}}$ each have a zero of order 1 , so adding the corresponding entries in table 9 we see that the Tate representation for $\Sigma^{\prime}$ is $\frac{1}{2}$ vect $\oplus \operatorname{spin}_{*}=\frac{1}{2} \rho_{0} \oplus \operatorname{spin}_{*}$. (We expect the $\frac{1}{2} \rho_{0}$ summand since this is a branch point for $\mathfrak{s o}(9)$.) Also, the expression for the discriminant of this example from table 14 shows that the components $\Sigma$ and $\Sigma^{\prime}$ have intersection multiplicity 2 .

Now we turn to representation theory to compute the actual matter representation. As indicated in table 15 , since $k=2$, the matter representation is

$$
\rho=\left.\left(\frac{1}{2}(\mathbf{1} \otimes \text { vect }) \oplus \frac{1}{2}\left(\text { fund } \otimes \operatorname{spin}_{*}\right)\right)\right|_{\mathfrak{s u}(2) \oplus \mathfrak{s o}(9)} .
$$

The first summand is $\frac{1}{2} \rho_{0}$ for $\mathfrak{s o}(9)$, and the second summand is prequaternionic, since fund is a quaternionic representation of $\mathfrak{s u}(2)$.

There are three things to check in order to verify anomaly cancellation:

1. When the matter representation is restricted to $\mathfrak{g}(\Sigma)=\mathfrak{s u}(2)$, the charged part is eight copies of the fundamental, since the spinor representation of $\mathfrak{s o}(9)$ has dimension 16 and we have half of that. This agrees with the Tate representation for $\Sigma$.

2. When restricted to $\mathfrak{g}\left(\Sigma^{\prime}\right)=\mathfrak{s o}(9)$, the charged part is $\frac{1}{2}$ vect $\oplus$ spin, since $\frac{1}{2}$ fund has dimension 1 as a representation of $\mathfrak{s u}(2)$. This agrees with the Tate representation for $\Sigma^{\prime}$.

3. To evaluate the representation-multiplicity, we use the scaling property of that quantity. Thus, for a half-representation, we get half of the representation-multiplicity of the corresponding full representation. In our case, since

$$
\begin{aligned}
& \operatorname{Tr}_{\text {fund }_{\mathfrak{s u}(2)}} F^{2}=\operatorname{tr}_{\mathfrak{s u}(2)} F^{2}, \\
& \operatorname{Tr}_{\text {spin }_{\mathfrak{s o}(9)}} F^{2}=4 \operatorname{tr}_{\mathfrak{s o}(9)} F^{2},
\end{aligned}
$$


we have $\mu_{\rho}\left(\mathfrak{g}(\Sigma), \mathfrak{g}\left(\Sigma^{\prime}\right)\right)=\frac{1}{2}(1 \cdot 4)=2$, and we see that the representation-multiplicity coincides with the intersection multiplicity.

Thus, the anomalies cancel.

A similar analysis applies after we make a basechange $t=s^{2}$, obtaining a representation for $\mathfrak{s u}(2) \oplus \mathfrak{s o}(10)$ with $k=1$. We omit the details.

- Example 18 has fibers of type $I_{2}$ along $\Sigma$ and fibers of type $I V^{*}$ along $\Sigma^{\prime}$, which meet at a common point. There is local monodromy along $\Sigma^{\prime}$, so the gauge algebra must be $\mathfrak{s u}(2) \oplus \mathfrak{f}_{4}$. According to table 14 , along $\Sigma, \gamma_{\Sigma}$ has a zero of order 14 , so the Tate representation for $\Sigma$ is 14 fund. On the other hand, along $\Sigma^{\prime}, \beta_{\Sigma^{\prime}}$ has a zero of order 3 , so that the Tate representation for $\Sigma^{\prime}$ is $\frac{3}{2} \mathbf{2 6}=\frac{1}{2} \rho_{0} \oplus \mathbf{2 6}$. (We expect the $\frac{1}{2} \rho_{0}$ summand since this is a branch point for $\mathfrak{f}_{4}$.) Also, the expression for the discriminant of this example from table 14 shows that the components $\Sigma$ and $\Sigma^{\prime}$ have intersection multiplicity 3 .

As indicated in table 15 , since $k=2$, and using the fact that $\left.\mathbf{2 7}\right|_{\mathfrak{f}_{4}}=$ $\mathbf{1} \oplus \mathbf{2 6}$, the matter representation is

$$
\rho=(\text { fund } \otimes \mathbf{1}) \oplus \frac{1}{2}(\mathbf{1} \otimes \mathbf{2 6}) \oplus \frac{1}{2}(\text { fund } \otimes \mathbf{2 6})
$$

The middle summand is $\frac{1}{2} \rho_{0}$ for $\mathfrak{f}_{4}$, and the last summand is prequaternionic, since fund is a quaternionic representation of $\mathfrak{s u}(2)$.

There are three things to check in order to verify anomaly cancellation:

1. When the matter representation is restricted to $\mathfrak{g}(\Sigma)=\mathfrak{s u}(2)$, the charged part is 14 copies of the fundamental, since the total dimension of the representations on the $\mathfrak{f}_{4}$ side is $2+26=28$ and we have half of that. This agrees with the Tate representation for $\Sigma$.

2. When restricted to $\mathfrak{g}\left(\Sigma^{\prime}\right)=\mathfrak{f}_{4}$, the charged part is $\frac{3}{2} \mathbf{2 6}$, since the total dimension of the representations on the $\mathfrak{s u}(2)$ side is $1+2=3$ and we have half of that. This agrees with the Tate representation for $\Sigma^{\prime}$.

3. To evaluate the representation-multiplicity, we again use the scaling property of that quantity to evaluate it for a half-representation. We have

$$
\begin{aligned}
\operatorname{Tr}_{\text {fund }_{\mathfrak{s u}(2)}} F^{2} & =\operatorname{tr}_{\mathfrak{s u}(2)} F^{2} \\
\operatorname{Tr}_{\mathbf{2 6}_{\mathfrak{f}_{4}}} & F^{2}=6 \operatorname{tr}_{\mathfrak{f}_{4}} F^{2}
\end{aligned}
$$


so that $\mu_{\rho}\left(\mathfrak{g}(\Sigma), \mathfrak{g}\left(\Sigma^{\prime}\right)\right)=\frac{1}{2}(1 \cdot 6)=3$. Hence, the representationmultiplicity coincides with the intersection multiplicity. Thus, the anomalies cancel.

A similar analysis applies after we make a basechange $t=s^{2}$, obtaining a representation for $\mathfrak{s u}(2) \oplus \mathfrak{e}_{6}$ with $k=1$. We omit the details.

- Example 19 has fibers of type $I_{3}$ along $\Sigma$ and fibers of type $I_{5}$ along $\Sigma^{\prime}$, which meet at a common point. There is local monodromy along both $\Sigma$ and $\Sigma^{\prime}$, so the gauge algebra must be $\mathfrak{s p}(1) \oplus \mathfrak{s p}(2)$. According to table 14 , along $\Sigma, \beta_{\Sigma}$ has a zero of order 3 and $\gamma_{\Sigma}$ has a zero of order 6 , so the Tate representation for $\Sigma$ is the sum of two terms from table 14, totaling $\frac{15}{2}$. fund $=\frac{1}{2} \rho_{0}+\frac{13}{2}$ fund. (We expect the $\frac{1}{2} \rho_{0}$ summand since this is a branch point for $\mathfrak{s p}(1)$; the remaining representation $\frac{13}{2}$ fund is pre-quaternionic.) On the other hand, along $\Sigma^{\prime}, \beta_{\Sigma^{\prime}}$ has a zero of order 3 and $\gamma_{\Sigma^{\prime}}$ has a zero of order 2 , so adding the corresponding entries in table 9 we see that the Tate representation for $\Sigma^{\prime}$ is $\frac{3}{2} \Lambda_{\text {irr }}^{2} \oplus$ $\frac{7}{2}$ fund $=\frac{1}{2} \rho_{0} \oplus \Lambda_{\text {irr }}^{2} \oplus \frac{5}{2}$ fund. (We expect the $\frac{1}{2} \rho_{0}$ summand since this is a branch point for $\mathfrak{s p}(4)$; the remaining representation $\Lambda_{\text {irr }}^{2} \oplus \frac{5}{2}$ fund is pre-quaternionic.)) Also, the expression for the discriminant of this example from table 14 shows that the components $\Sigma$ and $\Sigma^{\prime}$ have intersection multiplicity 2 .

Now we turn to representation theory to compute the actual matter representation. As indicated in table 15 , since $k=2$, and using the facts that

$$
\begin{aligned}
\text { fund }\left._{\mathfrak{s u}(3)}\right|_{\mathfrak{s p}(1)} & =\mathbf{1} \oplus \text { fund, } \\
\text { fund }\left._{\mathfrak{s u}(5)}\right|_{\mathfrak{s p}(2)} & =\mathbf{1} \oplus \text { fund, } \\
\left.\Lambda_{\mathfrak{s u}(5)}^{2}\right|_{\mathfrak{s p}(2)} & =\mathbf{1} \oplus \text { fund } \oplus \Lambda_{\text {irr }}^{2},
\end{aligned}
$$

the matter representation is the charged part of

$$
\left.\left(\frac{1}{2}(\mathbf{1} \otimes \text { fund }) \oplus \frac{1}{2}(\text { fund } \otimes \text { fund }) \oplus \frac{1}{2}\left(\text { fund } \otimes \Lambda^{2}\right)\right)\right|_{\mathfrak{s p}(1) \oplus \mathfrak{s p}(2)},
$$

which equals

$$
\frac{3}{2}(\mathbf{1} \otimes \text { fund }) \oplus(\text { fund } \otimes \mathbf{1}) \oplus(\text { fund } \otimes \text { fund }) \oplus \frac{1}{2}\left(\mathbf{1} \otimes \Lambda_{\text {irr }}^{2}\right) \oplus \frac{1}{2}\left(\text { fund } \otimes \Lambda_{\text {irr }}^{2}\right) .
$$

Subtracting $\frac{1}{2} \rho_{0}(\mathfrak{s p}(1))+\frac{1}{2} \rho_{0}(\mathfrak{s p}(2))=($ fund $\otimes \mathbf{1}) \oplus \frac{1}{2}\left(\mathbf{1} \otimes \Lambda_{\text {irr }}^{2}\right) \oplus(\mathbf{1} \otimes$ fund) leaves a pre-quaternionic representation. 
There are three things to check in order to verify anomaly cancellation:

1. When the matter representation is restricted to $\mathfrak{g}(\Sigma)=\mathfrak{s p}(1)$, the charged part is $1+\frac{1}{2} \operatorname{dim}\left(\Lambda_{\text {irr }}^{2}\right)+\operatorname{dim}\left(\right.$ fund $\left._{\mathfrak{s p}(2)}\right)=1+\frac{5}{2}+4=\frac{15}{2}$ copies of the fundamental. This agrees with the Tate representation for $\Sigma$.

2. When restricted to $\mathfrak{g}\left(\Sigma^{\prime}\right)=\mathfrak{s p}(2)$, the charged part is $\frac{3}{2}+$ $\operatorname{dim}\left(\right.$ fund $\left._{\mathfrak{s p}(1)}\right)=\frac{7}{2}$ copies of the fundamental representation plus $\frac{1}{2}+\frac{1}{2} \operatorname{dim}\left(\right.$ fund $\left._{\mathfrak{s p}(1)}\right)=\frac{3}{2}$ copies of $\Lambda_{\mathrm{irr}}^{2}$. This agrees with the Tate representation for $\Sigma^{\prime}$.

3. To evaluate the representation-multiplicity, we use the linearity property of that quantity, getting contributions from two different irreducible representations of $\mathfrak{s p}(1) \oplus \mathfrak{s p}(2)$. We have

$$
\begin{aligned}
& \operatorname{Tr}_{\text {fund }_{\mathfrak{s} \mathfrak{p}(1)}} F^{2}=\operatorname{tr}_{\mathfrak{s p}(1)} F^{2}, \\
& \operatorname{Tr}_{\text {fund }_{\mathfrak{s} \mathfrak{p}(2)}} F^{2}=\operatorname{tr}_{\mathfrak{s p}(2)} F^{2}, \\
& \operatorname{Tr}_{\Lambda_{\mathrm{irr}, \mathfrak{s} \mathfrak{p}(2)}^{2}} F^{2}=2 \operatorname{tr}_{\mathfrak{s p}(2)} F^{2},
\end{aligned}
$$

and so

$$
\begin{aligned}
\mu_{\frac{1}{2}\left(\text { fund } \otimes \Lambda_{\text {irr }}^{2}\right)}\left(\mathfrak{g}(\Sigma), \mathfrak{g}\left(\Sigma^{\prime}\right)\right) & =\frac{1}{2}(1 \cdot 2)=1, \\
\mu_{\text {fund } \otimes \text { fund }}\left(\mathfrak{g}(\Sigma), \mathfrak{g}\left(\Sigma^{\prime}\right)\right) & =1 \cdot 1=1,
\end{aligned}
$$

so the total representation-multiplicity is $1+1=2$. This coincides with the intersection multiplicity.

Thus, the anomalies cancel.

A similar analysis applies after we make a basechange $t=s^{2}$, obtaining a representation for $\mathfrak{s u}(3) \oplus \mathfrak{s u}(5)$ with $k=1$. We omit the details.

- Finally, Example 20 has fibers of type $I_{3}$ along $\Sigma_{+}$and $\Sigma_{-}$, and fibers of type $I_{2}$ along $\Sigma$, which all meet at a common point. There is no local or global monodromy for any of these, so the natural gauge algebra to consider is $\mathfrak{s u}(2) \oplus \mathfrak{s u}(3) \oplus \mathfrak{s u}(3)$. We need to calculate the Tate representation of each component, as well as the representation-multiplicity for each pair of components. Along $\Sigma, \gamma_{\Sigma}$ has a zero of order 10, so the Tate representation for $\Sigma$ is 10 fund. Along $\Sigma_{ \pm}, \beta_{\Sigma_{ \pm}}$has a zero of order 4 and $\gamma_{\Sigma_{ \pm}}$has a zero of order 7 , so the Tate representation for $\Sigma_{ \pm}$is

$$
\frac{4}{2} \text { fund }+7 \cdot \text { fund }=9 \cdot \text { fund }
$$


Also, from the form of the discriminant in table 14 we see that each pair of components from $\Sigma, \Sigma_{+}$, and $\Sigma_{-}$meets with intersection multiplicity 3.

Now from table 15 since $k=1$, the matter representation is

$$
\rho=(\mathbf{1} \otimes \text { fund } \otimes \text { fund }) \oplus(\text { fund } \otimes \mathbf{1} \otimes \mathbf{1}) \oplus(\text { fund } \otimes \text { fund } \otimes \text { fund }),
$$

as a representation of $\mathfrak{s u}(2) \oplus \mathfrak{s u}(3) \oplus \mathfrak{s u}(3)$. There are several things to check:

1. When the matter representation is restricted to $\mathfrak{g}(\Sigma)=\mathfrak{s u}(2)$, the charged part is $(1+9)$ copies of the fundamental representation, 1 from the middle term and 9 from the last term. This agrees with the Tate representation for $\Sigma$.

2. When restricted to $\mathfrak{g}\left(\Sigma_{ \pm}\right)=\mathfrak{s u}(3)$, the charged part is $(3+6)$ copies of the fundamental representation, with three copies coming from the first term and $2 \cdot 3$ copies coming from the last term. This agrees with the Tate representation for $\Sigma_{ \pm}$.

3. All of the representations here are fundamentals of $\mathfrak{s u}(m)$, so all of the ratios of traces are 1 . However, $\left(\Sigma, \Sigma_{ \pm}\right)$has three bifundamentals (coming from the last term), so the representation-multiplicity is 3 .

4. Similarly, $\left(\Sigma_{+}, \Sigma_{-}\right)$has three bifundamentals: one from the first term in $\rho$ and two from the last term, so the representation-multiplicity is again 3 .

Thus, the anomalies cancel.

\section{Discussion}

As the examples in the past two sections have shown, the geometry and representation theory conspire in wonderful ways to ensure anomaly cancellation in every case. One of the remarkable things about the present approach is how easy it is to calculate the various Tate cycles as well as the intersection multiplicites of pairs of divisors, starting from the Weierstrass equation. It is natural to wonder whether this data is sufficient to determine the matter representation itself.

That is, suppose we are expecting an actual pre-quaternionic representation at a given point. Whatever it is, it will be Casimir equivalent to some combination of the basic representations (such as adj, fund and $\Lambda^{2}$ in the $\mathfrak{s u}(m)$ case), but that combination would typically have both positive and 
negative coefficients. Could it be true that there is only one "honest" representation (up to complex conjugation of factors) in the Casimir equivalence class?

We already have an example in hand - Example 10 - which shows that this is too optimistic. But one could hope that the number of representations is small, and that the number of times there are duplications could be controlled in some fashion. This is a purely algebraic question about the representation ring and the sub-semiring generated by actual representations which deserves to be studied further. One might then hope to find an additional piece of geometric information which would distinguish among the allowed representations in cases of ambiguity.

There are a number of other ways that this work could be usefully extended. First, we assumed that the gauge algebra has no abelian summands, which geometrically corresponds to Mordell-Weil group of rank zero. It would be very interesting to study cases with abelian summands allowed. Some steps in this direction are taken in [58].

Second, although our formulation allows components of the discriminant locus to have singular points, we have not studied this case in detail. Some examples in addition to Sadov's appear in [22] but it would be good to have a more systematic treatment. For example, the representations of $\mathfrak{s u}(\mathrm{m})$ which we studied in detail here are all Casimir equivalent to combinations of fund and $\Lambda^{2}$ alone; allowing adj gives a much richer class of representations (and also necessarily implies that the relevant discriminant-component $\Sigma$ is singular).

And finally, although we made some comments about elliptic fibrations with higher-dimensional bases or whose total space is not Calabi-Yau, many of our results clearly extend to these settings and deserve a more systematic treatment there.

\section{Acknowledgment}

We would like to thank S. Katz, V. Kumar, K. Rubin, S. Schafer-Nameki, J. Silverman, J. Sully, Y. Tachikawa and especially W. Taylor for useful discussions. DRM thanks the Aspen Center for Physics for hospitality during various stages of preparation of this paper. Research partially supported by the National Science Foundation grants DMS-0606578, DMS-1007414, PHY-1066293 and by World Premier International Research Center Initiative (WPI Initiative), MEXT, Japan. 


\section{Appendix A. Notation and terminology from gauge theory}

Lemma Appendix A.1. Let $Y$ be a manifold equipped with a principal $G$-bundle $\mathcal{G}$, called the "gauge bundle":

(1) Each fiber $\operatorname{ad}(\mathcal{G})_{x}$ of $\operatorname{ad}(\mathcal{G})$ is isomorphic to the Lie algebra $\mathfrak{g}$ of $G$, with $\mathcal{G}_{x}$ acting on $\operatorname{ad}(\mathcal{G})_{x}$ via the adjoint action of $G$ on $\mathfrak{g}$.

(2) The curvature $F$ of the gauge connection is an $\operatorname{ad}(\mathcal{G})$-valued two-form.

(3) Similarly, if $Y$ is equipped with a (pseudo-)Riemannian metric, then the curvature $R$ of the Levi-Civita connection is a two-form taking values in the endomorphisms of the tangent bundle.

(4) Any representation $\rho$ of the Lie algebra can be regarded as a homomorphism $\rho: \mathfrak{g} \rightarrow \operatorname{End}(V)$ for some (complex) vector space $V$. As an endomorphism of $V, \rho\left(F_{x}\right)$ can be raised to the $k$ th power.

Proof. This can be derived, for example, from [49].

Definition Appendix A.1. We denote by

$$
\operatorname{Tr}_{\rho} F^{k}=\operatorname{trace}_{V} \rho(F)^{k}
$$

the trace of the resulting endomorphism $\rho\left(F_{x}\right)^{k}$ of $V$.

Definition Appendix A.2. Similarly,

$$
\operatorname{tr} R^{k}=\operatorname{trace}_{V} v(R)^{k}
$$

where $v$ is the "vector" representation of the corresponding orthogonal group.

These expressions do not depend on the choice of isomorphism to $\mathfrak{g}$, in fact they are invariant under the adjoint action of $G$ on $\mathfrak{g}$ and so is independent of choices. The above notation is then well defined.

\section{Appendix B. Tate's algorithm}

Kodaira's analysis identifies the type of singular fiber along each component of the discriminant locus, and this is almost enough to identify the gauge group. However, there is one additional piece of information, provided by Tate's algorithm, which specifies the monodromy of the family of 
Kodaira fibers over the component. That information has traditionally been presented in the form which Tate gave it - involving (generic) changes of coordinates in the Weierstrass (or Tate) model — but here we formulate the same information in a more intrinsic form.

Thus, we start with a Weierstrass equation

$$
y^{2}=x^{3}+f x+g
$$

and the associated discriminant ${ }^{25}$

$$
\Delta=4 f^{3}+27 g^{2}
$$

and seek conditions, which specify both the type of the fiber (following Kodaira) and the monodromy (following Tate). We regard $f$ and $g$ as elements of the ring $K[[z]]$ of formal power series in $z$ with coefficients in some specified field $K$. (For simplicity, we assume that $K$ contains the complex number field $\mathbb{C}$.) Typically, $z$ is a local parameter whose vanishing describes a divisor $\Sigma$ in some algebraic variety $B$, and $K$ is constructed from the field of rational functions on the algebraic variety by localization and taking residue field. Note that in $K[[z]]$ we are allowed to divide by any non-zero element which is not a multiple of $z$; this means that all results obtained through this algorithm only hold generically on the algebraic variety $B$, and may fail to hold in some particular coordinate systems.

To determine the desired conditions on $f, g$, and $\Delta$, we follow Tate's procedure described in Sections 7 and 8 of [40], specializing to the case of the coefficient field $K$ having characteristic zero, which allows us to simplify certain aspects of Tate's procedure. We follow the numbering of cases given in Tate's paper. We shall have occasion to use the discriminant for a generalized Weierstrass model

$$
Y^{2}=X^{3}+u X^{2}+v X+w,
$$

which is easily calculated from (4.2) to be

$$
\Delta=4 u^{3} w-u^{2} v^{2}-18 u v w+27 w^{2}
$$

(cf. [40, Section 1] or [10, Appendix I]).

\footnotetext{
${ }^{25}$ We use the normalization of the discriminant which is common in the F-theory literature.
} 
Case 1. If the fiber along $z=0$ is generically non-singular, then $z \backslash \backslash \Delta$. This is case $I_{0}$ in Kodaira's classification, represented by the first line of table 4 .

Case 2. If the fiber along $z=0$ is generically singular, we may assume that $z \mid \Delta$. In this case, there is a change of coordinates that translates the singular point in the fiber to the origin, and this puts the equation in the form

$$
Y^{2}=X^{3}+u X^{2}+v X+w
$$

with $z \mid v$ and $z \mid w$. (This change of coordinates can be taken to be $(x, y)=$ $(X+u / 3, Y)$ with $u=-9 g / 2 f$ or $u=2 f^{2} / 3 g$, at least one of which is guaranteed not to have a pole along $z=0$.) We write $v=v_{1} z$ and $w=w_{1} z$.

The condition to have type $I_{m}$ is then that $z \nmid u$ (which is easily seen to be equivalent to $z \not\{f$ or to $z \backslash \backslash g)$. In this case we define $m=\operatorname{ord}_{z=0}(\Delta)$ : this accounts for the next few lines of table 4 . Note that in this case, $u \equiv-9 g / 2 f$ $\bmod z$. The discriminant can be then expanded as follows:

$$
\Delta=4 u^{3} w_{1} z+O\left(z^{2}\right)
$$

Note that if $m=1$ then the total space is non-singular and there is nothing further to do. On the other hand, if $m \geq 2$ then $z^{2} \mid w$. If $m=2$ no monodromy is possible; if $m \geq 3$ we can write $w=w_{2} z^{2}$ and compute the discriminant again:

$$
\Delta=\left(4 u^{3} w_{2}-u^{2} v_{1}^{2}\right) z^{2}+O\left(z^{3}\right)
$$

Thus, if $m \geq 3$ we can write $w=\left(v_{1}^{2} / 4 u\right) z^{2}+w_{3} z^{3}$. Now, making the coordinate change $(X, Y)=\left(\widetilde{X}-\left(v_{1} / 2 u\right) z, \widetilde{Y}\right)$, we find a new equation of the form

$$
\widetilde{Y}^{2}=\widetilde{X}^{3}+\widetilde{u} \widetilde{X}^{2}+\widetilde{v}_{2} z^{2} \widetilde{X}+\widetilde{w}_{3} z^{3}
$$

valid for $I_{m}$ whenever $m \geq 3$. Note that $\widetilde{u} \equiv u \bmod z$.

Now let us resolve the singularities of the Weierstrass Equation (B.4); in an appropriate chart the first blowup has an exceptional divisor determined by the intersection of $z=0$ and the quadratic terms in Equation (B.4), namely,

$$
y_{2}^{2}=\left.\widetilde{u} x_{2}^{2}\right|_{z=0}
$$

and whether this exceptional divisor is reducible or irreducible is determined by the Tate monodromy relation, that is, is determined by whether $\left.\widetilde{u}\right|_{z=0}=$ 
$\left.u\right|_{z=0}=\left.(-9 g / 2 f)\right|_{z=0}$ has a square root in the field $K$ or not, or equivalently, whether the monodromy cover defined by

$$
\psi^{2}+\left.(9 g / 2 f)\right|_{z=0}=0
$$

is reducible or irreducible ${ }^{26}$. In Tate's original algorithm, the reducible case is called Case 2a, and the irreducible case is called Case 2b. This explains the monodromy entry in the fourth line of table 4.

Case 3. Now we may assume that $z \mid u$ (or equivalently that $z \mid f$ and $z \mid g$ ), and in this case it is convenient to return our attention to the original Weierstrass form (B.1). If $z^{2} \backslash \not g$, we have a Kodaira fiber of type $I I$ (the next line on the Table). There is no monodromy issue in this case.

Case 4. We next assume that in addition, $z^{2} \mid g$. At this point we need to start resolving the singularity; we can write $f=f_{1} z$ and $g=g_{2} z^{2}$ and on an appropriate chart of the blowup (with $x=z x_{1}$ and $y=z y_{1}$ ) we get an equation

$$
y_{1}^{2}=z x_{1}^{3}+f_{1} x_{1}+g_{2} .
$$

The exceptional divisor is defined by

$$
\left.\left(y_{1}^{2}=f_{1} x_{1}+g_{2}\right)\right|_{z=0}
$$

and this is absolutely irreducible if and only if $z \backslash f_{1}$, i.e., $z^{2} \backslash \nmid f$. This gives a Kodaira fiber of type $I I I$, the next line on the Table. There is again no monodromy issue.

Case 5. Now assume in addition $z^{2} \mid f$, which implies that $z^{3} \mid \Delta$. In this case our blown up equation is

$$
Y^{2}=z X^{3}+z f_{2} X+g_{2}
$$

and the exceptional divisor has equation

$$
Y^{2}=\left.g_{2}\right|_{z=0}
$$

Again we encounter a monodromy issue: the exceptional divisor is reducible if $\left.g_{2}\right|_{z=0}$ has a square root in the field $K$, and irreducible otherwise. Since

\footnotetext{
${ }^{26}$ The divisor is actually absolutely irreducible, that is, it is irreducible even after passing to an extension field $L$ of $K$. The point in this case is that when a quadratic equation in two variables has maximal rank, it is absolutely irreducible.
} 
$\left.g_{2}\right|_{z=0}=\left.\left(g / z^{2}\right)\right|_{z=0}$, this can be expressed as before in terms of a monodromy cover; the one we need this time is

$$
\psi^{2}-\left.\left(g / z^{2}\right)\right|_{z=0}=0 .
$$

We have have Kodaira type $I V$ exactly when $z^{3} \backslash g$.

Cases 6 and beyond. If we now assume in addition that $z^{3} \mid g$, we have arrived at a Weierstrass equation of the form

$$
y^{2}=x^{3}+f_{2} z^{2} x+g_{3} z^{3} .
$$

The first blowup now leads to an equation of the form

$$
z y_{2}^{2}=x_{1}^{3}+f_{2} x_{1}+g_{3},
$$

with exceptional divisor

$$
z=0=x_{1}^{3}+f_{2} x_{1}+g_{3}
$$

and Tate now describes the algorithm as having three branches, depending on the number of roots of (B.6).

First Branch: Case 6. If (B.6) has three distinct roots, then we have type $I_{0}^{*}$. This is the case in which $\operatorname{ord}_{z=0}(\Delta)=6$, and the further behavior is determined by the behavior of the splitting field of that polynomial. In our Table, we have rephrased this as the behavior of the monodromy cover defined by

$$
\psi^{3}+\left(f / z^{2}\right)_{z=0} \psi+\left.\left(g / z^{3}\right)\right|_{z=0}=0 .
$$

Second Branch: Case 7. When (B.6) has one simple root and one double root, we will get Kodaira type $I_{m-4}^{*}$ for $m=\operatorname{ord}_{z=0}(\Delta)-2$. There is a monodromy issue here as well, and to fully determine things, we need to execute several blowups. Tate describes these by means of a subprocedure. To initialize the subprocedure, we make a change of coordinates similar to Case 1 , but this time putting the double root of (B.6) at $X=0$. This coordinate change can be done via $(x, y)=\left(X+u_{1} z / 3, Y\right)$, with $u_{1}=-9 g_{3} / 2 f_{2}=-9 g / 2 z f$ or $u_{1}=2 f_{2}^{2} / 3 g_{3}$, at least one of which is guaranteed not to have a pole along $z=0$. After the coordinate change, $v$ and $w$ each vanish to an additional order. We write $v=v_{3} z^{3}$ and $w=w_{4} z^{4}$, giving an equation of the form

$$
Y^{2}=X^{3}+u_{1} z X^{2}+v_{3} z^{3} X+w_{4} z^{4} .
$$

Subprocedure. We assume that we are in the second branch, so that $z \nmid u_{1}$. We claim by induction on the integer $\mu \geq 5$ that if $\operatorname{ord}_{z=0}(\Delta) \geq \mu+2$ 
then, possibly after a change of coordinates, the Equation (B.7) can be chosen so that $z^{[(\mu+1) / 2]} \mid v$ and $z^{\mu-1} \mid w$. The initial step $\mu=5$ of this induction is what we established in the paragraph above. In the course of carrying out the induction, we will also exhibit various blowups of the singularity and arrive at the monodromy condition.

It is easiest to divide the analysis of the inductive step into two cases: $\mu$ odd and $\mu$ even. To handle the first case, we assume $\mu=2 n-1, n \geq 3$, and write the equation in the form

$$
Y^{2}=X^{3}+u_{1} z X^{2}+v_{n} z^{n} X+w_{2 n-2} z^{2 n-2} .
$$

We compute the discriminant in this case as

$$
\Delta=4 u_{1}^{3} w_{2 n-2} z^{2 n+1}+O\left(z^{2 n+2}\right) .
$$

One coordinate chart in the blowup has $X=z^{n-1} X_{n-1}, Y=z^{n-1} Y_{n-1}$ and the equation takes the form

$$
Y_{n-1}^{2}=z^{n-1} X_{n-1}^{3}+u_{1} z X_{n-1}^{2}+v_{n} z X_{n-1}+w_{2 n-2},
$$

with exceptional divisor

$$
\left.\left(Y_{n-1}^{2}=w_{2 n-2}\right)\right|_{z=0} .
$$

If $\operatorname{ord}_{z=0}(\Delta)>\mu+2$ then we see from Equation (B.9) that $z \mid w_{2 n-2}$. In this case, the exceptional divisor is a double line, and the equation already has the form specified in our inductive statement for $\mu+1$, so we have established the induction in this case. On the other hand, if $z \backslash w_{2 n-2}$ then there is a monodromy issue: (B.11) is reducible if and only if $\left.w_{2 n-2}\right|_{z=0}$ is a square. From Equation (B.9) we see that we can write

$$
\left.w_{2 n-2}\right|_{z=0}=\left.\frac{\Delta}{4 z^{2 n+1} u_{1}^{3}}\right|_{z=0}=\left.\frac{1}{4}\left(\frac{\Delta}{z^{2 n+1}}\right)\left(\frac{-2 z f}{9 g}\right)^{3}\right|_{z=0}
$$

and this is the form in which we expressed the monodromy cover condition $\psi^{2}=\left.w_{2 n-2}\right|_{z=0}$ in table 4.

We now consider the case in which $\mu$ is even, and let $\mu=2 n, n \geq 3$. We write the equation in the form

$$
Y^{2}=X^{3}+u_{1} z X^{2}+v_{n} z^{n} X+w_{2 n-1} z^{2 n-1} .
$$


We compute the discriminant in this case as

$$
\Delta=\left(-u_{1}^{2} v_{n}^{2}+4 u_{1}^{3} w_{2 n+3}\right) z^{2 n+2}+O\left(z^{2 n+3}\right) .
$$

One coordinate chart in the blowup has $X=z^{n-1} X_{n-1}, Y=z^{n} Y_{n}$ and the equation takes the form

$$
z Y_{n}^{2}=z^{n-1} X_{n-1}^{3}+u_{1} X_{n-1}^{2}+v_{n} X_{n-1}+w_{2 n-1},
$$

with exceptional divisor

$$
0=\left.\left(u_{1} X_{n-1}^{2}+v_{n} X_{n-1}+w_{2 n-1}\right)\right|_{z=0}
$$

If $\operatorname{ord}_{z=0}(\Delta)>\mu+2$ then we see from Equation (B.13) that $z \mid\left(-v_{n}^{2}+\right.$ $\left.4 u_{1} w_{2 n-1}\right)$, so we may write

$$
w_{2 n-1}=\frac{v_{n}^{2}}{4 u_{1}}+w_{2 n} z .
$$

Then the equation takes the form

$$
Y^{2}=X^{3}+u_{1} z\left(X+\frac{1}{2} v_{n} z^{n-1}\right)^{2}+w_{2 n} z^{2 n}
$$

Making the coordinate change $(X, Y)=\left(\widetilde{X}-\frac{1}{2} v_{n} z^{n-1}, \tilde{Y}\right)$ then puts the equation in the form specified for $\mu+1$, so the induction is established.

On the other hand, if $\operatorname{ord}_{z=0}(\Delta)=\mu+2$ then the exceptional divisor (B.15) is reducible if and only if the discriminant $\left.\left(v_{n}^{2}-4 u_{1} w_{2 n-1}\right)\right|_{z=0}$ of the quadratic Equation (B.15) is a perfect square. Note that, by Equation (B.13), we can write

$$
\left.\left(v_{n}^{2}-4 u_{1} w_{2 n-1}\right)\right|_{z=0}=\left.\frac{-\Delta}{z^{2 n+2} u_{1}^{2}}\right|_{z=0}=\left.\left(\frac{-\Delta}{z^{2 n+2}}\right)\left(\frac{-2 z f}{9 g}\right)^{2}\right|_{z=0}
$$

and this is the form in which we expressed the monodromy cover condition $\psi^{2}=\left.\left(v_{n}^{2}-4 u_{1} w_{2 n-1}\right)\right|_{z=0}$ in table 4 .

Branch 3 begins. Case 8: Now suppose that (B.6) has a triple root. In this case, $z \mid u_{1}$ and it is again convenient to return our attention to the original Weierstrass form (B.1). We have $z^{3} \mid f$ and $z^{4} \mid g$, and can write the 
equation in the form

$$
y^{2}=x^{3}+f_{3} z^{3} x+g_{4} z^{4} .
$$

The key chart for the blowup is

$$
y_{2}^{2}=z^{2} x_{2}^{3}+f_{3} z x+g_{4}
$$

When $z \backslash g_{4}$ we have Kodaira type $I V^{*}$, and in this case there is a monodromy issue: the exceptional divisor is described by

$$
\left.\left(y_{2}^{2}=g_{4}\right)\right|_{z=0}
$$

which is reducible exactly when $\left.g_{4}\right|_{z=0}=\left.\left(g / z^{4}\right)\right|_{z=0}$ is a perfect square. This leads to the final "monodromy cover" entry in table 4.

Branch 3 continues. Case 9: We now assume ${ }^{27} z^{5} \mid g$. The relevant chart for the blowup is

$$
z y_{3}^{2}=z x_{2}^{3}+f_{3} x_{2}+g_{5}
$$

If $z \backslash f_{3}$ then we have Kodaira type $I I I^{*}$. There is no monodromy issue in this case.

Branch 3 continues. Case 10: We now assume that $z \mid f_{3}$, i.e., $z^{4} \mid f$. If $z^{6} \backslash \mathrm{g}$, we get Kodaira type $I I^{*}$ with no monodromy. If $z^{6} \mid g$, the original Weierstrass equation was not minimal. We should start over with

$$
y_{3}^{2}=x_{2}^{3}+f_{4} x_{2}+g_{6}
$$

\section{Appendix C. Casimir computations for $\mathfrak{s o}(\ell)$.}

For $\mathfrak{s o}(\ell)$ with $\ell=2 m$ or $\ell=2 m+1$, the weight lattice has generators $\varepsilon_{1}$, $\ldots, \varepsilon_{m}$. The Weyl group $\mathcal{W}_{\mathfrak{s o}(\ell)}$ acts by permutations and sign changes on the $\varepsilon_{i}^{\prime}$ 's, with $\mathcal{W}_{\mathfrak{s o}(2 m+1)}=\mathfrak{S}_{m} \rtimes\left(\mathbb{Z}_{2}\right)^{m}$ while $\mathcal{W}_{\mathfrak{s o}(2 m)}=\mathfrak{S}_{m} \rtimes\left(\mathbb{Z}_{2}\right)^{m-1}$ where the product of all signs must be 1 in the second case. As is easily seen, the $\mathcal{W}$-invariant polynomials are generated by the elementary symmetric functions in $\varepsilon_{1}^{2}, \ldots, \varepsilon_{m}^{2}$ in the case of $\mathfrak{s o}(2 m+1)$, while these are supplemented by $\prod_{i} \varepsilon_{i}$ in the case of $\mathfrak{s o}(2 m)$ (and in this latter case, $\sigma_{m}\left(\varepsilon_{1}^{2}, \ldots, \varepsilon_{m}^{2}\right)=\prod_{i} \varepsilon_{i}^{2}$ becomes superfluous).

\footnotetext{
${ }^{27}$ Note that right at this point, Tate's paper has a small typographical error concerning the exponent of $z$, which he calls $\pi$.
} 
The vector representation has weights

$$
\varepsilon_{1}, \ldots, \varepsilon_{m},-\varepsilon_{1}, \ldots,-\varepsilon_{m}
$$

for $\mathfrak{s o}(2 m)$, and

$$
\varepsilon_{1}, \ldots, \varepsilon_{m}, 0,-\varepsilon_{1}, \ldots,-\varepsilon_{m}
$$

for $\mathfrak{s o}(2 m+1)$. Thus, in both cases, we have

$$
\operatorname{Tr}_{\text {vect }} F^{k}=\sum\left(\varepsilon_{i}\right)^{k}+\sum\left(-\varepsilon_{i}\right)^{k}=2 \sum \varepsilon_{i}^{k}
$$

for every even $k$. Our convention is to set $\operatorname{tr}=\frac{1}{2} \operatorname{Tr}_{\mathrm{vect}}$, so that

$$
\operatorname{tr}\left(F^{k}\right)=\sum \varepsilon_{i}^{k}
$$

Now in the case of $\mathfrak{s o}(2 m+1)$, the spinor representation has weights $\frac{1}{2}\left( \pm \varepsilon_{1} \pm \cdots \pm \varepsilon_{m}\right)$, whereas for $\mathfrak{s o}(2 m)$, the even (resp. odd) spinor representation has weights $\frac{1}{2}\left( \pm \varepsilon_{1} \pm \cdots \pm \varepsilon_{m}\right)$ where the total number of minus signs is even (resp. odd).

The Casimir operators evaluate as follows:

$$
\operatorname{Tr}_{\operatorname{spin} *} F^{k}=\frac{1}{2^{k}} \sum_{a_{1}, \ldots, a_{m}}\left((-1)^{a_{1}} \varepsilon_{1}+\cdots(-1)^{a_{m}} \varepsilon_{m}\right)^{k},
$$

which we only consider for $k$ even. In the sum $a_{i} \in \mathbb{Z}_{2}$, and in the case of $\mathfrak{s o}(2 m)$ we impose the condition $(-1)^{\sum a_{i}}= \pm 1$ to distinguish the two spinor representations. In particular, the number of terms in the sum is equal to the dimension of $\operatorname{spin} *$. Note that these expressions are all invariant under the Weyl group.

Taking a binomial expansion of these expressions in any case with $\ell \geq 5$ other than $\ell=8$, the expansion of $\operatorname{Tr}_{\text {spin } *} F^{2}$ can only involve $\sum_{i} \varepsilon_{i}^{2}$ and that of $\operatorname{Tr}_{\mathrm{spin} *} F^{4}$ can only involve $\sum_{i} \varepsilon_{i}^{4}$ and $\sum_{i<j} \varepsilon_{i}^{2} \varepsilon_{j}^{2}$. The reason $\ell=8$ is special is that in that case, $\prod_{i} \varepsilon_{i}$ is also possible for $k=4$. In any event, the implication is that aside from the $\ell=8, k=4$ case, any terms with odd powers of $\varepsilon_{i}$ will cancel out.

It follows that

$$
\operatorname{Tr}_{\text {spin } *} F^{2}=\frac{1}{4} \sum_{a_{1}, \ldots, a_{m}}\left(\sum_{i} \varepsilon_{i}^{2}\right)=\frac{1}{4} \operatorname{dim}(\operatorname{spin} *) \operatorname{tr}\left(F^{2}\right),
$$


and that for $\ell \neq 8$,
(C.1) $\operatorname{Tr}_{\text {spin } *} F^{4}=\frac{1}{16} \sum_{a_{1}, \ldots, a_{m}}\left(\frac{4 !}{4 !} \sum_{i} \varepsilon_{i}^{4}+\frac{4 !}{2 ! 2 !} \sum_{i<j} \varepsilon_{i}^{2} \varepsilon_{j}^{2}\right)$,

$$
\begin{aligned}
& =\frac{1}{16} \operatorname{dim}(\operatorname{spin} *)\left(\operatorname{tr}\left(F^{4}\right)+6 \cdot \frac{1}{2}\left(\left(\operatorname{tr}\left(F^{2}\right)^{2}\right)-\left(\operatorname{tr}\left(F^{4}\right)\right)\right)\right) \\
& =\frac{1}{16} \operatorname{dim}(\operatorname{spin} *)\left(3\left(\operatorname{tr}\left(F^{2}\right)^{2}\right)-2\left(\operatorname{tr}\left(F^{4}\right)\right) .\right.
\end{aligned}
$$

The case $\ell=8$ is special, because we get a term

$$
\frac{4 !}{1 ! 1 ! 1 ! 1 !}(-1)^{a_{1}+a_{2}+a_{3}+a_{4}} \varepsilon_{1} \varepsilon_{2} \varepsilon_{3} \varepsilon_{4}
$$

which evaluates to $24 \prod_{i} \varepsilon_{i}$ for $\operatorname{spin}_{+}$and $-24 \prod_{i} \varepsilon_{i}$ for spin_. Thus, for $\ell=8$ (using the fact that $\operatorname{dim}(\operatorname{spin} *)=8$ ) we have

$$
\operatorname{Tr}_{\text {spin }_{ \pm}} F^{4}=\frac{3}{2}\left(\operatorname{tr}\left(F^{2}\right)^{2}\right)-\left(\operatorname{tr}\left(F^{4}\right)\right) \pm 12\left(\prod_{i} \varepsilon_{i}\right)
$$

The key relation for us is:

$$
\operatorname{Tr}_{\text {vect }} F^{4}+\operatorname{Tr}_{\text {spin }_{+}} F^{4}+\operatorname{Tr}_{\text {spin. }} F^{4}=3\left(\operatorname{tr}\left(F^{2}\right)^{2}\right)
$$

which follows immediately since $\operatorname{Tr}_{\text {vect }} F^{4}=2 \operatorname{tr}\left(F^{4}\right)$.

For completeness, we also work out the adjoint representation of $\mathfrak{s o}(\ell)$. This time the weights are $\pm \varepsilon_{i} \pm \varepsilon_{j}$ for $i<j$, and when $\ell$ is odd, also $\pm \varepsilon_{i}$. We thus have

$$
\begin{aligned}
\operatorname{Tr}_{\text {adjoint }}\left(F^{2}\right)= & \sum_{i<j}\left(\left(\varepsilon_{i}+\varepsilon_{j}\right)^{2}+\left(\varepsilon_{i}-\varepsilon_{j}\right)^{2}\right. \\
& \left.+\left(-\varepsilon_{i}+\varepsilon_{j}\right)^{2}+\left(-\varepsilon_{i}-\varepsilon_{j}\right)^{2}\right), \\
& +(\ell-2 m) \sum_{i}\left(\varepsilon_{i}^{2}+\left(-\varepsilon_{i}\right)^{2}\right), \\
= & \sum_{i<j}\left(4 \varepsilon_{i}^{2}+4 \varepsilon_{j}^{2}\right)+2(\ell-2 m) \sum_{i} \varepsilon_{i}^{2},
\end{aligned}
$$




$$
\begin{aligned}
& =2 \sum_{i \neq j}\left(\varepsilon_{i}^{2}+\varepsilon_{j}^{2}\right)+2(\ell-2 m) \sum_{i} \varepsilon_{i}^{2}, \\
& =4(m-1) \sum_{i} \varepsilon_{i}^{2}+2(\ell-2 m) \sum_{i} \varepsilon_{i}^{2}, \\
& =(2 \ell-4) \operatorname{tr}\left(F^{2}\right),
\end{aligned}
$$

and

$$
\begin{aligned}
\operatorname{Tr}_{\text {adjoint }}\left(F^{4}\right)= & \sum_{i<j}\left(\left(\varepsilon_{i}+\varepsilon_{j}\right)^{4}+\left(\varepsilon_{i}-\varepsilon_{j}\right)^{4}+\left(-\varepsilon_{i}+\varepsilon_{j}\right)^{4}+\left(-\varepsilon_{i}-\varepsilon_{j}\right)^{4}\right) \\
& +(\ell-2 m) \sum_{i}\left(\varepsilon_{i}^{4}+\left(-\varepsilon_{i}\right)^{4}\right), \\
\text { C.11) } & \sum_{i<j}\left(4 \varepsilon_{i}^{4}+24 \varepsilon_{i}^{2} \varepsilon_{j}^{2}+4 \varepsilon_{j}^{4}\right)+2(\ell-2 m) \sum_{i} \varepsilon_{i}^{4}, \\
= & 24 \sum_{i<j} \varepsilon_{i}^{2} \varepsilon_{j}^{2}+2 \sum_{i \neq j}\left(\varepsilon_{i}^{4}+\varepsilon_{j}^{4}\right)+2(\ell-2 m) \sum_{i} \varepsilon_{i}^{4}, \\
\text { C.13) }= & 12\left(\left(\sum_{i} \varepsilon_{i}^{2}\right)^{2}-\sum_{i} \varepsilon_{i}^{4}\right) \\
& +4(m-1) \sum_{i} \varepsilon_{i}^{4}+2(\ell-2 m) \sum_{i} \varepsilon_{i}^{4}, \\
= & 12\left(\operatorname{tr}\left(F^{2}\right)^{2}\right)+(2 n-16) \operatorname{tr}\left(F^{4}\right) .
\end{aligned}
$$

Note that all terms with odd powers of $\varepsilon_{i}$ and $\varepsilon_{j}$ canceled explicitly in the second line of the computation. In particular, this computation is valid for all values of $\ell$ including $\ell=8$.

\section{References}

[1] E. Witten, String theory dynamics in various dimensions, Nucl. Phys. B 443 (1995), 85-126, arXiv:hep-th/9503124.

[2] P. S. Aspinwall, Enhanced gauge symmetries and K3 surfaces, Phys. Lett. B 357 (1995), 329-334, arXiv:hep-th/9507012.

[3] P. S. Aspinwall and M. Gross, The $\mathrm{SO}(32)$ heterotic string on a K3 surface, Phys. Lett. B 387 (1996), 735-742, arXiv:hep-th/9605131. 
[4] M. Bershadsky, K. Intriligator, S. Kachru, D. R. Morrison, V. Sadov and C. Vafa, Geometric singularities and enhanced gauge symmetries, Nucl. Phys. B 481 (1996), 215-252, arXiv:hep-th/9605200.

[5] K. Intriligator, D. R. Morrison and N. Seiberg, Five-dimensional supersymmetric gauge theories and degenerations of Calabi-Yau spaces, Nucl. Phys. B 497 (1997), 56-100, arXiv:hep-th/9702198.

[6] E. Witten, New "gauge" theories in six dimensions, J. High Energy Phys. 01 (1998), 001, arXiv:hep-th/9710065.

[7] D. -E. Diaconescu and R. Entin, Calabi-Yau spaces and fivedimensional field theories with exceptional gauge symmetry, Nucl. Phys. B 538 (1999), 451-484, arXiv:hep-th/9807170.

[8] S. Katz and C. Vafa, Matter from geometry, Nucl. Phys. B 497 (1997), 146-154, arXiv:hep-th/9606086.

[9] P. S. Aspinwall, S. Katz and D. R. Morrison, Lie groups, Calabi-Yau threefolds, and F-theory, Adv. Theor. Math. Phys. 4 (2000), 95-126, arXiv: hep-th/0002012.

[10] A. Grassi and D. R. Morrison, Group representations and the Euler characteristic of elliptically fibered Calabi-Yau threefolds, J. Algebraic Geom. 12 (2003), 321-356, arXiv:math.AG/0005196.

[11] V. Kumar and W. Taylor, String universality in six dimensions, arXiv:0906.0987 [hep-th].

[12] (2009), 050, arXiv:0910.1586 [hep-th].

[13] V. Kumar, D. R. Morrison and W. Taylor, Mapping $6 D N=1$ supergravities to F-theory, J. High Energy Phys. 02 (2010), 099, arXiv:0911.3393 [hep-th].

[14] Global aspects of the space of $6 D N=1$ supergravities, J. High Energy Phys. 11 (2010), 118, arXiv:1008.1062 [hep-th].

[15] V. Kumar, D. Park and W. Taylor, 6D supergravity without tensor multiplets, arXiv:1011.0726 [hep-th].

[16] F. Denef, Lectures on constructing string vacua, in String Theory and the Real World: From Particle Physics to Astrophysics, ed. C. Bachas, École d'été de physique des Houches, session LXXXVII, Elsevier, 2008, pp. 483-610, arXiv:0803.1194 [hep-th]. 
[17] J. J. Heckman, Particle physics implications of F-theory, Annu. Rev. Nucl. Part. Sci. 60 (2010), 237-265, arXiv:1001. 0577 [hep-th].

[18] P. Candelas, D. -E. Diaconescu, B. Florea, D. R. Morrison and G. Rajesh, Codimension-three bundle singularities in F-theory, J. High Energy Phys. 06 (2002), 014, arXiv:hep-th/0009228.

[19] F. Denef, M. R. Douglas, B. Florea, A. Grassi and S. Kachru, Fixing all moduli in a simple F-theory compactification, Adv. Theor. Math. Phys. 9 (2005), 861-929, arXiv:hep-th/0503124.

[20] J. H. Schwarz, Anomaly-free supersymmetric models in six dimensions, Phys. Lett. B 371 (1996), 223-230, arXiv: hep-th/9512053.

[21] S. Katz, D. R. Morrison, S. Schäfer-Nameki and J. Sully, Tate's algorithm and F-theory, J. High Energy Phys. 08 (2011), 094, arXiv:1106.3854 [hep-th].

[22] D. R. Morrison and W. Taylor, Matter and singularities, J. High Energy Phys. 01 (2012), 022, arXiv:1106.3563 [hep-th].

[23] M. Esole and S. -T. Yau, Small resolutions of SU(5)-models in F-theory, arXiv: 1107.0733 [hep-th].

[24] C. Chevalley, Sur la classification des algèbres de Lie simples et de leurs représentations, C. R. Acad. Sci. Paris 227 (1948), 1136-1138.

[25] Harish-Chandra, On some applications of the universal enveloping algebra of a semisimple Lie algebra, Trans. Amer. Math. Soc. 70 (1951), 28-96.

[26] C. W. Bernard, N. H. Christ, A. H. Guth and E. J. Weinberg, Pseudoparticle parameters for arbitrary gauge groups, Phys. Rev. D 16(3) (1977), 2967-2977.

[27] J. Erler, Anomaly cancellation in six dimensions, J. Math. Phys. 35 (1994), 1819-1833, arXiv:hep-th/9304104.

[28] P. van Nieuwenhuizen, Anomalies in quantum field theory: cancellation of anomalies in $d=10$ supergravity, Leuven Notes in Mathematical and Theoretical Physics. Series B: Theoretical Particle Physics, vol. 3, Leuven University Press, Leuven, 1988.

[29] N. Nakayama, On Weierstrass models, Algebraic geometry and commutative algebra, Vol. II, Kinokuniya, 1988, pp. 405-431. 
[30] A. Grassi, Divisors on elliptic Calabi-Yau 4-folds and the superpotential in F-theory, I, J. Geom. Phys. 28 (1998), 289-319, arXiv: alg-geom/9704008.

[31] _ Log contractions and equidimensional models of elliptic threefolds, J. Algebraic Geom. 4 (1995), 255-276, arXiv:alg-geom/ 9305003.

[32] D. R. Morrison and M. R. Plesser, Non-spherical horizons, I, Adv. Theor. Math. Phys. 3 (1999), 1-81, arXiv:hep-th/9810201.

[33] D. R. Morrison and C. Vafa, Compactifications of F-theory on Calabi-Yau threefolds, II, Nucl. Phys. B 476 (1996), 437-469, arXiv: hep-th/9603161.

[34] P. Deligne, Courbes elliptiques: formulaire (d'après J. Tate), Modular functions of one variable, IV (Proc. Int. Summer School, Univ. Antwerp, Antwerp, 1972), Lecture Notes in Math., 476, Springer, Berlin, 1975, pp. $53-73$.

[35] I. Dolgachev and M. Gross, Elliptic threefolds. I. Ogg-Shafarevich theory, J. Algebraic Geom. 3 (1994), 39-80, arXiv:alg-geom/ 9210009.

[36] M. Gross, A finiteness theorem for elliptic Calabi-Yau threefolds, Duke Math. J. 74 (1994), 271-299, arXiv:alg-geom/9305002.

[37] J. de Boer, R. Dijkgraaf, K. Hori, A. Keurentjes, J. Morgan, D. R. Morrison, and S. Sethi, Triples, fluxes, and strings, Adv. Theor. Math. Phys. 4 (2001), 995-1186, arXiv:hep-th/0103170.

[38] P. S. Aspinwall and D. R. Morrison, Non-simply-connected gauge groups and rational points on elliptic curves, J. High energy Phys. 07 (1998), 012, arXiv:hep-th/9805206.

[39] K. Kodaira, On compact analytic surfaces. II, III, Ann. Math. 77(2) (1963), 563-626, 78 (1963) 1-40.

[40] J. Tate, Algorithm for determining the type of a singular fiber in an elliptic pencil, Modular functions of one variable, IV (Proc. Int. Summer School, Univ. Antwerp, Antwerp, 1972), Lecture Notes in Math., 476, Springer, Berlin, 1975, pp. 33-52.

[41] H. Cohen, A course in computational algebraic number theory, Graduate Texts in Mathematics, 138, Springer, Berlin, 1993. 
[42] R. Donagi and M. Wijnholt, Model building with F-theory, arXiv:0802.2969 [hep-th].

[43] C. Beasley, J. J. Heckman and C. Vafa, GUTs and exceptional branes in F-theory - I, J. High energy Phys. 01 (2009), 058, arXiv:0802. 3391 [hep-th].

[44] E. Witten, Phase transitions in $M$-theory and F-theory, Nucl. Phys. B 471 (1996), 195-216, arXiv: hep-th/9603150.

[45] R. Slansky, Group theory for unified model building, Phys. Rept. 79 (1981), 1-128.

[46] W. G. McKay and J. Patera, Tables of dimensions, indices, and branching rules for representations of simple Lie algebras, Lecture Notes in Pure and Applied Mathematics, 69, Marcel Dekker Inc., New York, 1981.

[47] R. Miranda, The moduli of Weierstrass fibrations over $\mathbf{P}^{1}$, Math. Ann. 255 (1981), 379-394.

[48] E. Brieskorn, Singular elements of semi-simple algebraic groups, Actes du Congrès International des Mathématiciens (Nice, 1970), Tome 2, Gauthier-Villars, 1971, pp. 279-284.

[49] P. Slodowy, Simple singularities and simple algebraic groups, Lecture Notes in Mathematics, 815, Springer, Berlin, 1980.

[50] E. B. Dynkin, Semisimple subalgebras of semisimple Lie algebras, Mat. Sbornik N.S. 30(72) (1952), 349-462, English translation in AMS Transl ser. 2, 6 (1957) 111-244.

[51] K. Oguiso and T. Shioda, The Mordell-Weil lattice of a rational elliptic surface, Comment. Math. Univ. St. Paul. 40 (1991), 83-99.

[52] M. B. Green, J. H. Schwarz, and P. C. West, Anomaly free chiral theories in six-dimensions, Nucl. Phys. B 254 (1985), 327-348.

[53] M. B. Green and J. H. Schwarz, Anomaly cancellation in supersymmetric $D=10$ gauge theory and superstring theory, Phys. Lett. B 149 (1984), 117-122.

[54] A. Sagnotti, A note on the Green-Schwarz mechanism in open string theories, Phys. Lett. B 294 (1992), 196-203, arXiv:hep-th/9210127. 
[55] V. Sadov, Generalized Green-Schwarz mechanism in F-theory, Phys. Lett. B 388 (1996), 45-50, arXiv: hep-th/9606008.

[56] A. Grassi, On minimal models of elliptic threefolds, Math. Ann. 290 (1991), 287-301.

[57] S. Katz and D. R. Morrison, Gorenstein threefold singularities with small resolutions via invariant theory for Weyl groups, J. Algebr. Geom. 1 (1992), 449-530, arXiv:alg-geom/9202002.

[58] D. S. Park and W. Taylor, Constraints on 6D supergravity theories with abelian gauge symmetry, arXiv:1110.5916 [hep-th].

Department of Mathematics

University of Pennsylvania

PhilADELPHIA, PA 19104

USA

E-mail address: grassi@math.upenn.edu

Institute for the Physics and Mathematics of the Universe

The UnIVERSITY OF TOKYO

Kashiwa, ChiBa 277-8582

JAPAN

AND

Departments of Mathematics and Physics

UNIVERSITY OF CALIFORNIA

SANTA BARBARA, CA 93106

USA

E-mail address: drm@math.ucsb.edu

Received September 20, 2011 
